\title{
Copyright
}

by

Nicholas Hill Matlis

2006 
The Dissertation Committee for Nicholas Hill Matlis

certifies that this is the approved version of the following dissertation:

\section{Frequency Domain Holography of Laser Wakefields}

Committee:

Michael Downer, Supervisor

Manfred Fink

John Keto

Wendell Horton

Michael Becker

Boris Breizmann 


\title{
Frequency Domain Holography of Laser Wakefields
}

by

Nicholas Hill Matlis, B.A.

\author{
Dissertation \\ Presented to the Faculty of the Graduate School of \\ The University of Texas at Austin \\ in Partial Fulfillment \\ of the Requirements \\ for the Degree of
}

Doctor of Philosophy

The University of Texas at Austin

August 2006 
I dedicate this dissertation to Mom and Dad, who's belief in me never wavered, and to my grandfather with whom I wish this could have been shared 


\section{Acknowledgments}

I would like to thank my advisor Mike Downer for the opportunity to work in his lab. His eagerness to accept me as a student was a breath of new life in my graduate career at a time of difficulty when one advisor had gone, and my degree of tenure was making it challenging to find another. Dr. Downer has always fostered an environment of curiosity and exploration in his lab, encouraging new students to take up the mantle of responsibility in the lab, and pardoning them for the inevitable mistakes of judgement and pains of growth that every experimentalist faces. I believe I have learned something new every day in his lab, not only from the personal scientific freedom afforded me, but also in great measure from the opportunity to collaborate with and learn from my excellent colleagues and lab mates. I could not imagine a better place, or a better advisor to prepare a student to become an independent researcher. Even at his busiest, Dr. Downer has always found time to answer a question and provide advice. More importantly, he has always found a way to provide a means for his students to gain the crucial experience of working on important experiments. Most of all, I look up to Dr. Downer for his attitude of tolerance and patient persistence in both handling his students, gently guiding them along the right path, extracting the most out of them, and in pursing science, with excellent vision, never losing sight of the goal or sacrificing meticulous attention to detail. I am happy to be able to count Dr. Downer not just as an advisor, but also as a scientific mentor. Thank you Mike. 
I would like to thank my fellow students and lab mates both former and current from whom I have learned equally as much as from my own efforts. I thank Paul LeBlanc and Erhard Gaul for being my mentors in the lab, and showing me how things are done right. I thank Andy Rundquist for teaching me labVIEW and how to include diagrams and figures in my lab notebooks. I thank Rafal Zgadzaj, Bonggu Shim and Franklin Grigsby, with whom I have had the most contact for stimulating discussions on all manner of subjects (including physics), for their sharp minds and even sharper wits, for their comeraderie, and most of all, for learning with me and helping me learn with them how to work together towards a common objective. I thank the other members of the lab: Ben Bowes, Jinhee Kwon, Pete Figliozzi, Ramon Carriles-Jaimes, PJ Smith, Liangfeng Sun, Peng Dong, YongQiang An and Lucy-Marie Mantese (and others whos names recede into distant memory) for being my second family in the lab. I consider myself exceptionally fortunate to have had these awesome people with whom to share in this experience: part-adventure, part-ordeal, part-saga that has occupied more than one third of my life, and on whom to count among my friends. To those of you who graduate with me: "Congratulations!!!! We made it!" To those who are not quite there yet, take it from an ex-cynic: it will happen (eventually).

I would like to thank my committee members for their wisdom and excellent advice throughout the years, and for agreeing to be part of my committee.

I would like to thank my collaborators Anatoly Maksimchuk, Victor Yanovsky, Takeshi Matsuoka, Stephan Bulanov, Vladimir Chvykov, Galina Kalintchenko, Pascal Rousseau, and especially Steve Reed for allowing me to work with them on their amazing laser to produce these wonderful results.

I would like to thank my family for getting me here. Mom, Dad, Eric, Anthony and Sean. 
Nicholas Hill Matlis

The University of Texas at Austin

August 2006 


\title{
Frequency Domain Holography of Laser Wakefields
}

\author{
Publication No.
}

Nicholas Hill Matlis, Ph.D.

The University of Texas at Austin, 2006

Supervisor: Michael Downer

In this dissertation, single-shot measurement of longitudinal and transverse structure of resonantly driven laser wakefields by Frequency Domain Holography (FDH) is demonstrated. Visualization of laser wakefield structure is crucial for understanding the complex dynamics of the mutual interaction between the driving pulse, wakefield density oscillations and accelerated electron bunch. This diagnostic therefor provides a necessary tool for researchers to learn how to control and fine tune the interaction to produce electron beams with desirable properties. Wakefields were measured in a $2 \mathrm{~mm}$ Helium gas jet, for electron densities between $1 \times 10^{18}$ and 6 $\mathrm{x} 10^{18} \mathrm{~cm}^{-3}$, and laser pulses of $30 \mathrm{fs}$ duration and peak powers between $10 \mathrm{TW}$ and 30 TW. Detailed structural features of the wakefields, including radial extent of the wake, longitudinal wake development, and wavefront curvature, as well as 
features of the accompanying ionization front and plasma column are resolved. Results are compared with PIC simulations, and show a high degree of correspondence. Weaknesses of the current implementation of the technique and extensions to other parameter regimes are discussed. 


\section{Contents}

Acknowledgments $\quad$ v

Abstract viii

$\begin{array}{llr}\text { Chapter } 1 & \text { Introduction } & 1\end{array}$

Chapter 2 Setting up the Experiment 4

2.1 Generation and Characterization of FDH Pulses . . . . . . . . . 4

2.1 .1 Probe and Reference Bandwidth . . . . . . . . . 8

2.1.2 Probe-Reference Pulse Separation . . . . . . . . . . . . . . 10

2.1.3 Probe and Reference Chirp . . . . . . . . . . . . 12

2.1 .4 Pulse Duration . . . . . . . . . . . . . . . 14

2.2 Temporal Overlap f . . . . . . . . . . . . . . . . . 17

2.2 .1 Crude Overlap . . . . . . . . . . . . . . . 17

2.2 .2 Spatial Interferometry . . . . . . . . . . . . . 19

2.2 .3 Ionization Front Signature . . . . . . . . . . . . . 23

2.3 Spatial Overlap . . . . . . . . . . . . . . . . 25

2.3.1 Overlapping the Pump and Probe . . . . . . . . . 26

2.3 .2 Ionization-induced Defocusing . . . . . . . . . . . 28

2.4 Density Determination . . . . . . . . . . . . . . . . 31

2.4 .1 Phase Retrieval . . . . . . . . . . . . . . . . 32 
2.4 .2 Abel's Inversion $\ldots \ldots \ldots$

2.4.3 Adjusting the Density . . . . . . . . . . . . . . . . 38

2.5 Probe Imaging Setup . . . . . . . . . . . . . . . . . . . . . 38

2.5.1 Choosing the Magnification . . . . . . . . . . . 38

2.5.2 Calibrating the Spatial Dimension of the Spectrometer . . . . 41

2.5.3 Field of View . . . . . . . . . . . . . . . . . . 43

2.5.4 Choosing the Object Plane . . . . . . . . . . . . 43

2.6 Spectrometer Setup . . . . . . . . . . . . . . . . 45

$\begin{array}{lll}\text { Chapter } 3 & \text { Chirped Frequency Domain Holography } & 47\end{array}$

3.1 Designing the probe . . . . . . . . . . . . . . . 48

3.2 The reconstruction process f . . . . . . . . . . . . . 49

3.3 measuring probe chirp ...................... 51

Chapter 4 Wakefield Measurements $\quad 54$

4.1 Initial Attempt with Photon Acceleration . . . . . . . . . . . . . 54

4.2 First Wakefield Measurements, 11/21/05 . . . . . . . . . . . . . 59

4.3 First 30 TW shots, $11 / 22 / 05 \ldots \ldots \ldots \ldots$

$4.412 / 13 / 05$ Wakefields . . . . . . . . . . . . . . . 67

4.5 Simulation and Analysis . . . . . . . . . . . . . . . 73

4.5.1 Wakefield Morphology . . . . . . . . . . . . . . . . 74

4.5.2 Wakefield Amplitude . . . . . . . . . . . . . . 76

4.5.3 Density gradients in the gas profile . . . . . . . . . 81

$\begin{array}{lll}\text { Chapter } 5 & \text { Conclusions and Future Directions } & 85\end{array}$

5.1 potential modifications . . . . . . . . . . . 86

$\begin{array}{lr}\text { Bibliography } & 86\end{array}$

$\begin{array}{lr}\text { Bibliography } & 87\end{array}$ 
Vita 


\section{Chapter 1}

\section{Introduction}

In the beginning, there was light...

The field of laser wakefield acceleration began in 1979 with the publication of the now famous article by Tajima and Dawson entitled "Laser Electron Accelerator" [1]. At this time, it had been recognized by various researchers that the laser, with its ability to generate extremely high energy densities, presented the potential for creating a particle accelerator with very high field gradients and thus compact, very efficient acceleration. Although there were various concepts that were proposed to implement these high fields, the proposal of Tajima and Dawson to use a plasma as the intermediary to transfer energy between the laser field and the electrons has proven to be one of the most fruitful. An intense laser pulse propagating through a plasma excites a plasma wave known as a "Langmuir Wave" via the ponderomotive potential of the laser field. These waves have zero group velocity, but have a phase velocity equal to the group velocity of the laser pulse in the plasma, allowing them to keep up with the driving pulse and to accelerate charged particles. Although the electron beams generated by this process have typically been of poor quality (in terms of their energy and divergence characteristics), recent advances in this field have yielded beams of quality approaching that of a conventional RF accelerator 
$[2,3,4]$.

Tabletop laser-plasma accelerators driven by intense ultrashort laser pulses or charged-particle bunches can now produce GeV-range electron beams with $\lesssim$ $5 \%$ energy spread and $\lesssim 5$ mrad divergence. These beams can be used to generate femtosecond x-ray and gamma-ray pulses, providing compact radiation sources for application in medicine, nuclear engineering, materials science and high-energy physics. The accelerating fields approach $\sim 100 \mathrm{GeV} / \mathrm{m}$, which is thousands of times stronger than achievable in conventional accelerators. The morphology of these accelerating structures propagating behind the driving pulse is very sensitive to the parameters of the laser pulse and of the interaction. Simulations show that they can vary from nicely formed, sinusoidal waves with flat wavefronts in the linear regime, to spiked waves with curved wavefronts in the weakly non-linear regime, to cavities devoid of electrons in the "Bubble" or "Blow-out" regime. In many cases there is a dynamic evolution of the structure as the laser propagates. Because of their microscopic size and luminal velocity, the critical features of these structures and their evolution that determine energy, energy spread, collimation and charge of the accelerated beam have eluded direct single-shot observation, inhibiting progress in producing high quality beams and in correlating beam properties with wake structure. Here, single-shot visualization of laser wakefield accelerator (LWFA) structures is demonstrated for the first time, using Frequency Domain Holography (FDH), a technique designed to image structures propagating near light speed. The holographic snapshots capture the evolution of multiple wake periods, and resolve wavefront curvature seen previously only in simulations. These previously invisible features underlie wave breaking, electron injection and focusing within the wake, the key determinants of beam quality. Wake morphology is reconstructed in real-time, providing experimental feedback and optimization. Beyond accelerators, it is anticipated that FDH will provide an unprecedented visualization and feedback capability 
for structures created in diverse laser- and particle-plasma interactions, including fast ignition for laser fusion and plasma-based amplification and compression of ultra-intense laser pulses. 


\section{Chapter 2}

\section{Setting up the Experiment}

The primary elements involved in setting up a Frequency Domain Holography experiment are as follows: 1. generation and characterization of optical probe pulses, 2. temporal overlapping of probe and pump pulses, 3. spatial overlapping of probe and pump beams at the focus, 4. characterization of the target, 5. imaging of the interaction plane to a spectrometer, and 6. optimization of the spectrometer. A simplified schematic of the experimental setup is shown in figure 2.1.

\subsection{Generation and Characterization of FDH Pulses}

For Frequency Domain Holography, what is needed are two chirped pulses of approximately 1 ps duration, (with enough bandwidth to support 20 fs temporal resolution), separated by at least 2 ps. The mode should be quite small, in order to keep the focused spot size large. For this experiment, the focal length of the parabola was $1 \mathrm{~m}$, so we used a probe beam size of $\lesssim 8 \mathrm{~mm}$.

The apparatus for generating these pulses is very simple and easy to setup, contributing to FDH's attractiveness as a diagnostic. As shown in the experimental layout (figure 2.2), the $800 \mathrm{~nm}$ probe line first traverses a thin doubling crystal 


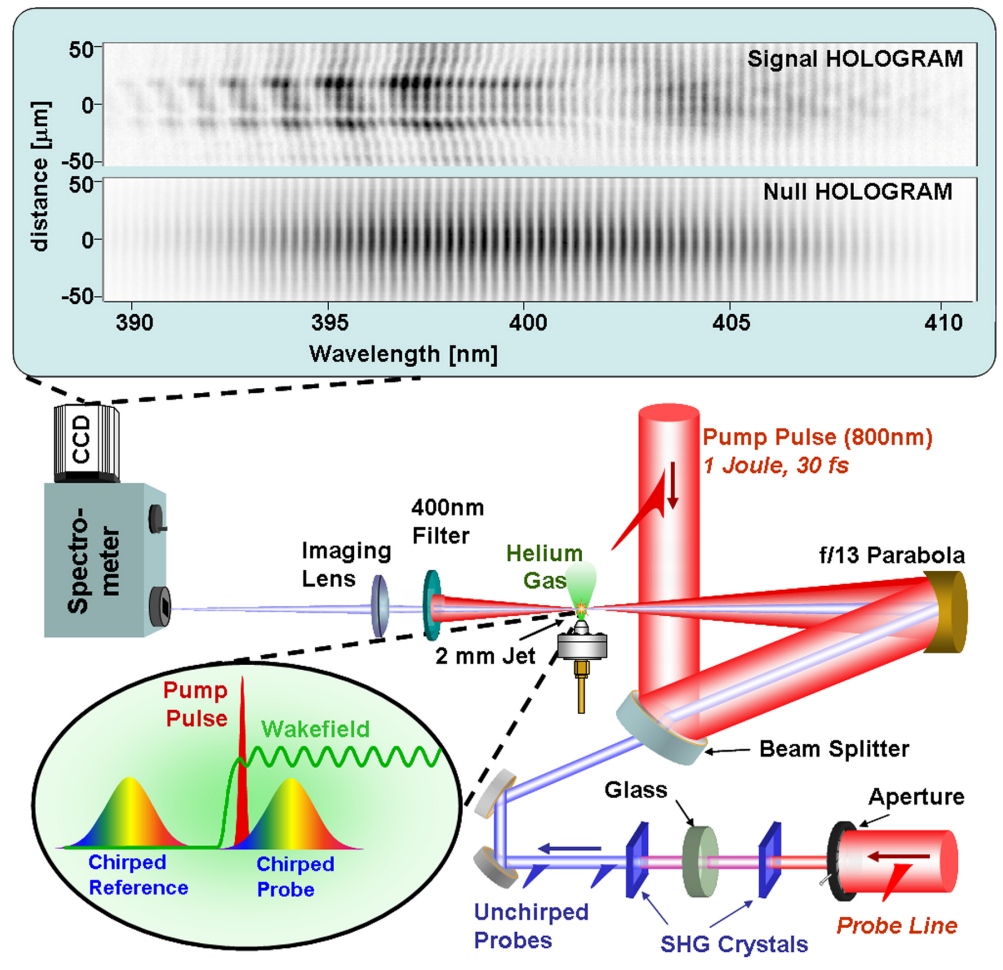

Figure 2.1: Experimental Layout 
(200 $\mu \mathrm{m}$ KDP, type I), up-converting a small percentage of the pulse into the second harmonic. The remaining, nearly undepleted fundamental then co-propagates with the second harmonic through a thick $(\sim 0.5 ")$ piece of glass, causing the red and the blue pulses to separate temporally (longitudinally) via group velocity dispersion. Upon traversing a second doubling crystal (also $200 \mu \mathrm{m}$ KDP, type I) the temporally-advanced fundamental now generates another second harmonic pulse, which precedes the first one. The fundamental is then picked off for use in a transverse shearing interferometer for measuring the interaction density. The remaining two $400 \mathrm{~nm}$ pulses are separated in time by about 2 picosecond, but traveling perfectly collinear to each other. The fabrication of the diagnostic probes for FDH is completed by chirping these pulses to the desired duration of about 1 picosecond, and combining them with the pump beam so that they can spatially overlap at the focus in a collinear geometry. Fortunately, both the chirping and the recombining can be done at once. In this experiment, as shown in figure $(* * ? * *)$, there is a dielectric high-reflector that serves as a final turning-mirror for the pump line before the parabolic focusing optic. This dielectric, though not intended for this use, serves as a reasonably good dichroic beam-splitter, reflecting $800 \mathrm{~nm}$ S-polarized light and transmitting $400 \mathrm{~nm}$ P-polarized light. By passing the probe line through this optic, the probe can be spatially overlapped and made collinear with the pump beam, and simultaneously, the probe pulses will be chirped by group velocity dispersion in the BK7 glass of the 1" thick optic.

In general, the "inline interferometer" described here is easy to build and has excellent performance characteristics. The fact that both pulses are generated in a collinear geometry (i.e. both pulses experience exactly the same optics), ensures that the probe-reference alignment is superb and also that both pulses will experience the same spatial phase artifacts, eliminated a potential source of phase noise. The fact that the two doubling crystals are thin (of comparable thickness) and up-convert 


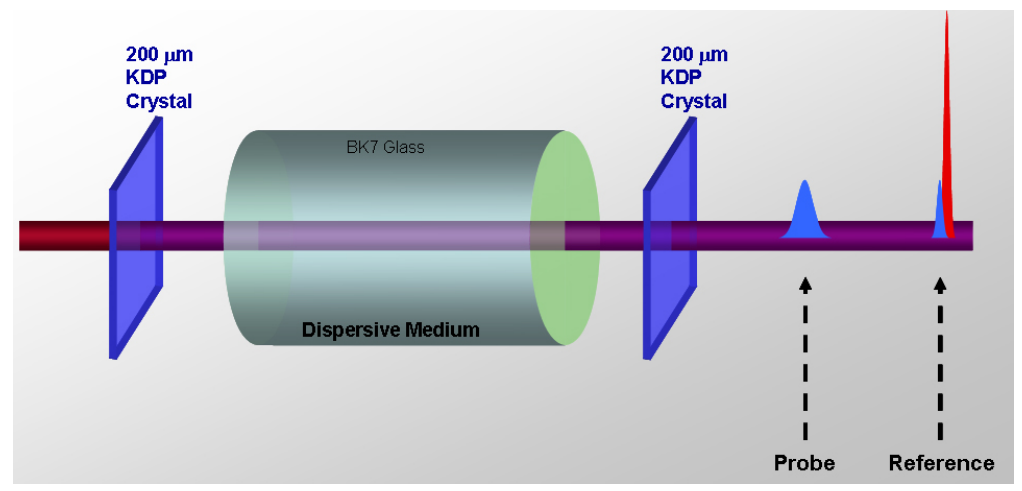

Figure 2.2: Setup for inline interferometer

only a small percentage of the fundamental, ensures that the intensities of the two probes are very well matched. All these factors contribute to the generation of very good interferograms. (** figure of null interferogram with good fringes**) "Very good" here means that the interference fringes will be straight, the fringe contrast will be high, and the background phase noise will be low, so that information encoded in the interference pattern can be faithfully retrieved.

This being said, there are, however, a few issues to consider when building an inline interferometer to ensure that these conditions are met. First, good doubling crystals must be chosen. These should have a high conversion efficiency, large phase-matching bandwidth and good surface quality. These qualities are all interdependent. In order to pass the full bandwidth of the fundamental, (which is desirable to optimize the bandwidth of the probes), the doubling crystal must be cut fairly thin (sub-mm in general), which causes the conversion efficiency to be low, and the surface quality to be affected. For conversion of $800 \mathrm{~nm}$ pulses, Potassium Di-Phosphate (KDP) Type I is usually a good choice. 


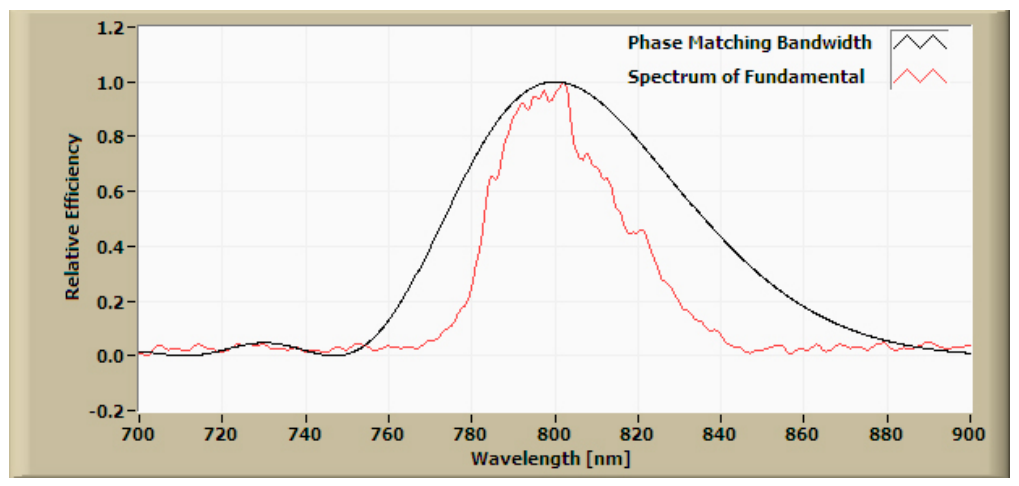

Figure 2.3: caption here

\subsubsection{Probe and Reference Bandwidth}

The bandwidth of the converted pulses is an important issue because it limits the maximum achievable temporal resolution of the measurement. (** calculation of bandwidth ${ }^{* *}$ )

where the relative efficiency is given by:

$$
\epsilon=\frac{\sin (\Delta k L / 2)}{\Delta k L / 2}
$$

where $\mathrm{L}$ is the crystal thickness, and $\Delta k$ is the phase mismatch, given by:

$$
\Delta k=2 \frac{2 \pi}{\lambda}\left[n_{o}(\omega)-n_{e}(2 \omega, \theta)\right]
$$

and $n_{o}(\omega)$ is the ordinary index at the fundamental and $n_{e}(2 \omega, \theta)$ is the extraordinary index (at angle $\theta$ with respect to the optic axis) at the second harmonic.

The conversion efficiency is important because there is usually a limited amount fundamental light available to produce the probe pulses. There must be sufficient probe light produced to saturate the detector in spite of having a very loose focus and lossy optics. It is desirable that the probe be generated without the 


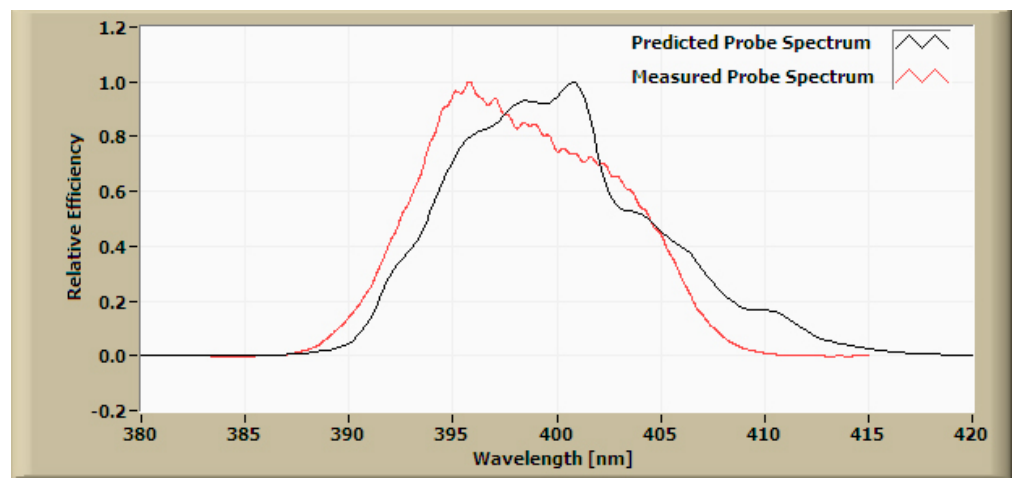

Figure 2.4: caption here

use of cumbersome optics to resize the beam and potentially adversely affect the beam quality. Surface quality of the thin crystal is also of great importance. Poor surface quality can change the divergence of the beam, causing the location of the probe focus to be different from that of the pump, if a common focusing element is used. This focal displacement will result in a lack of beam collimation of the probe at the interaction plane, and may ultimately cause distortions in the measurements. Poor surface quality can also lead to a distortion of the focused mode of the probe, mixing radial elements of the mode and resulting in phase fronts that are not smooth. Lack of smooth phase fronts can then lead to irregular fringes in the interference fringes and once again to distortions in the measurements. To address this issue, several crystals were characterized by determining their effect on the focused mode of the pump beam. (** images of effect of crystal on mode and on interferograms **) Inevitably, there are always some residual distortions. To compensate for these effects, an aperture was used far upstream on the probe line. Strong aperturing of the mode reduces the deleterious effects of crystal-surface unevenness by causing only a small portion of the crystal to be sampled. In addition, only the central airy disk of the diffracted beam contributes significantly to the signal, so the phase profile is made smoother. (** image effect of aperturing on interferogram quality 


\subsubsection{Probe-Reference Pulse Separation}

The temporal separation between the probe pulse and the reference pulse is determined by the group delay dispersion between the $800 \mathrm{~nm}$ fundamental, and the $400 \mathrm{~nm}$ second harmonic probe in the glass spacer. Thus the thickness of the glass spacer must be chosen so that the pulse separation is long enough to allow the probe and reference to be fully separated, but short enough that the fringes in the spectrometer are still easily resolvable. Resolvable means that there are multiple CCD pixels per fringe, and that the fringes are also considerably wider than the spectral image of the spectrometer slit on the CCD. Full separation of the probe and reference means that there is no temporal overlap of the two pulses. This is important, because for the reference to be effective, it must be temporally situated in a region free of disturbances from the interaction. In other words, the reference must precede the ionization front and wakefield so that these phenomena do not affect its phase. Ideally, the pulse separation should be very large, so that the relative delay between the probe and the pump (and therefore the plasma disturbance) can be varied to allow examination of temporal dynamics on a timescale larger than the duration of the probe without worrying about overlapping the plasma disturbance and the reference pulse.

In practice, as mentioned above, spectral resolution in the spectrometer is the limiting factor in this separation, and for these experiments the upper limit was about 3 - 4 picoseconds. The group delay dispersion is calculated by knowing the group velocity:

$$
\Delta t_{\text {Group Delay }}=\Delta t_{800 \mathrm{~nm}}-\Delta t_{400 \mathrm{~nm}}=\frac{L_{\text {glass }}}{v_{g}(800 \mathrm{~nm})}-\frac{L_{\text {glass }}}{v_{g}(400 \mathrm{~nm})}
$$

where $v_{g}$ is the group velocity, and $L_{\text {glass }}$ is the thickness of the glass. In 


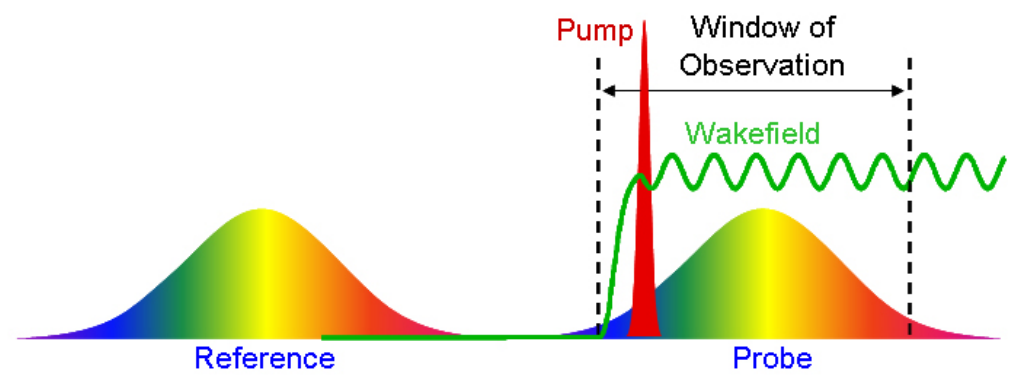

Figure 2.5: caption here

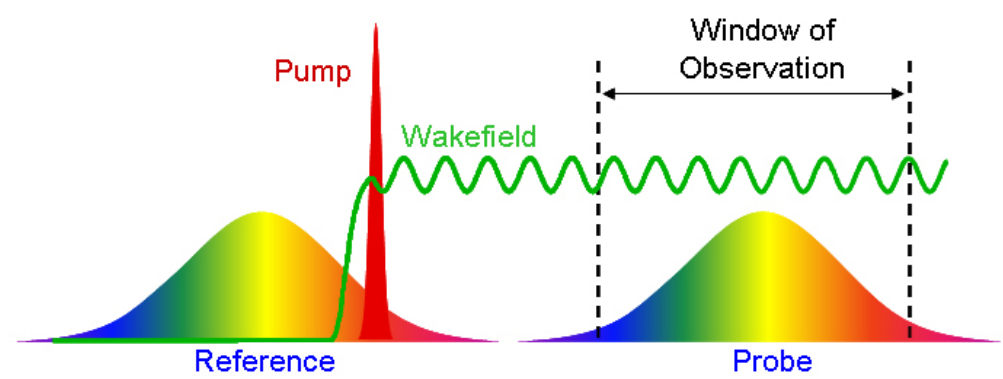

Figure 2.6: caption here 
particular, for a piece of BK7 glass of thickness $L_{B K 7}=0.5 "$, the pulse separation will be $\Delta t_{\text {Group Delay }} \cong 2.4 \mathrm{ps}$. It is important to note that because probe and reference pulses are passing through the glass spacer as different colors, any wedge to the spacer will induce an angular offset between them, which can potentially cause the two beams to not overlap at the focus.

\subsubsection{Probe and Reference Chirp}

The chirping of the probe and reference pulses is achieved by passing them through the material of the recombining beam splitter. It is one of the attractive features of this diagnostic that the temporal broadening of the probe pulses that inevitably results from the need to couple the probe beam to the pump beam through an optic becomes a desired effect. Because linear dispersion in glass is so strong at the second harmonic, the material used for the high reflectors of the pump beam is quite effective for chirping the probe. In this experiment there were actually two beam splitters that contributed to the stretching of the probe: the pick-off mirror for the transverse shearing interferometer $\left(3 / 8^{\prime \prime}\right.$ thick BK7, at $\left.45^{\circ}\right)$, and the pump high-reflector that was used to recombine the pump and probe (1" thick BK7, at $\left.45^{\circ}\right)$.

The total amount of material traversed by the probe pulse before reaching the interaction point is thus $52.2 \mathrm{~mm}$ of BK7, including the 1 " and the $3 / 8$ " beam splitters at $45^{\circ}$, and the $1 / 2$ " of BK7 at normal incidence from the glass spacer. The reference passes though the glass spacer as $800 \mathrm{~nm}$ light, so the contribution to the dispersion from the latter is roughly 20 times less than for the probe pulse. Therefore, ignoring the spacer, the reference traverses approximately $39.5 \mathrm{~mm}$ of BK7 glass. A reasonable estimate to the chirped pulse duration can be made by calculating the difference in the group delay experienced by photons with wavelengths at either end of the full width half max of the spectrum. 


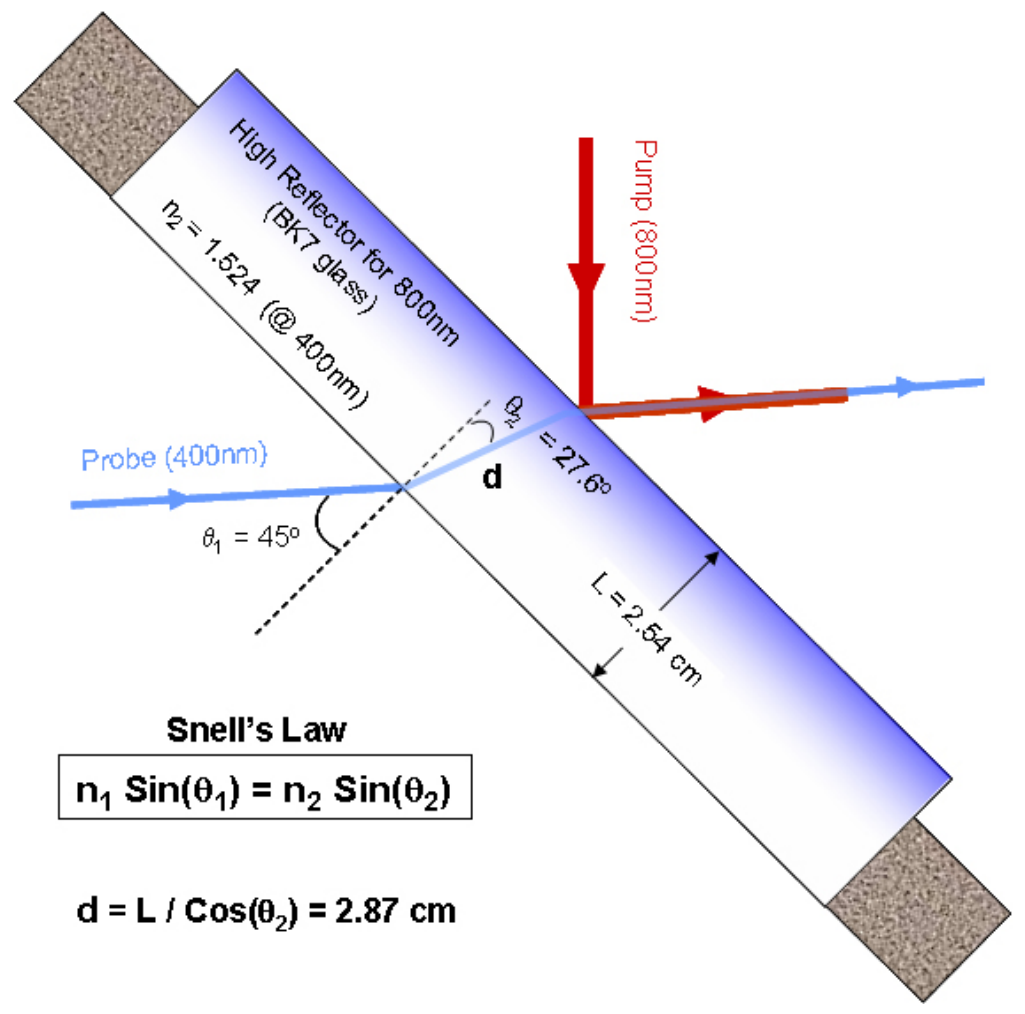

Figure 2.7: Close-up of recombining beam splitter

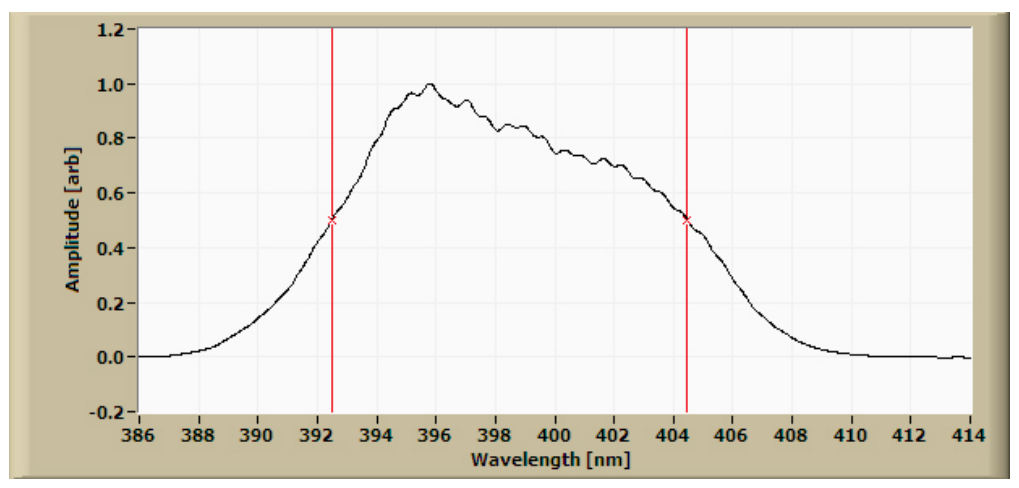

Figure 2.8: caption here 
The full-width-half-max points on the probe spectrum shown above are $\lambda_{F W H M}^{+}=392.5 \mathrm{~nm}$ and $\lambda_{F W H M}^{-}=404.5 \mathrm{~nm}$. The approximate chirped-pulse duration can then be calculated as:

$$
\tau_{\text {chirped }}=\frac{L_{\text {glass }}}{v_{g}\left(\lambda_{F W H M}^{+}\right)}-\frac{L_{\text {glass }}}{v_{g}\left(\lambda_{F W H M}^{-}\right)}
$$

The estimated chirped-probe duration is then $\tau_{p r} \sim 0.9 \mathrm{ps}$, while the chirpedreference duration is $\tau_{r e f} \sim 0.7 \mathrm{ps}$.

\subsubsection{Pulse Duration}

As mentioned above, the desired pulse duration is about $1 \mathrm{ps}$, and the desired probereference separation is about 2 ps or greater. In order to verify these experimental parameters, a third-order cross-correlator was set up. The basic concept is to take the pump pulse (at the fundamental), and the probe pulses (at the second harmonic), which are already spatially overlapped, and mix them in a BBO crystal to generate third harmonic, according to:

$$
E(3 \omega)=\chi_{i j} E_{i}^{p r b}(2 \omega)^{2} E_{j}^{p u m p}(\omega)
$$

The $3^{r d}$ harmonic intensity is then sensitive to the relative delay, and takes the form of a cross-correlation:

$$
I_{3 \omega}(t)=\int_{-\infty}^{\infty} I_{2 \omega}(\tau) I_{\omega}(\tau-t) d \tau
$$

Since the pump temporal profile is very short ( $30 \mathrm{fs}$ ) compared to that of the chirped probes, the third-harmonic intensity as a function of delay approximates the temporal profile of the probe-reference pulse train. I.e. $I_{\omega}(t) \approx \delta(t)$, so $I_{3 \omega}(t) \approx$ 


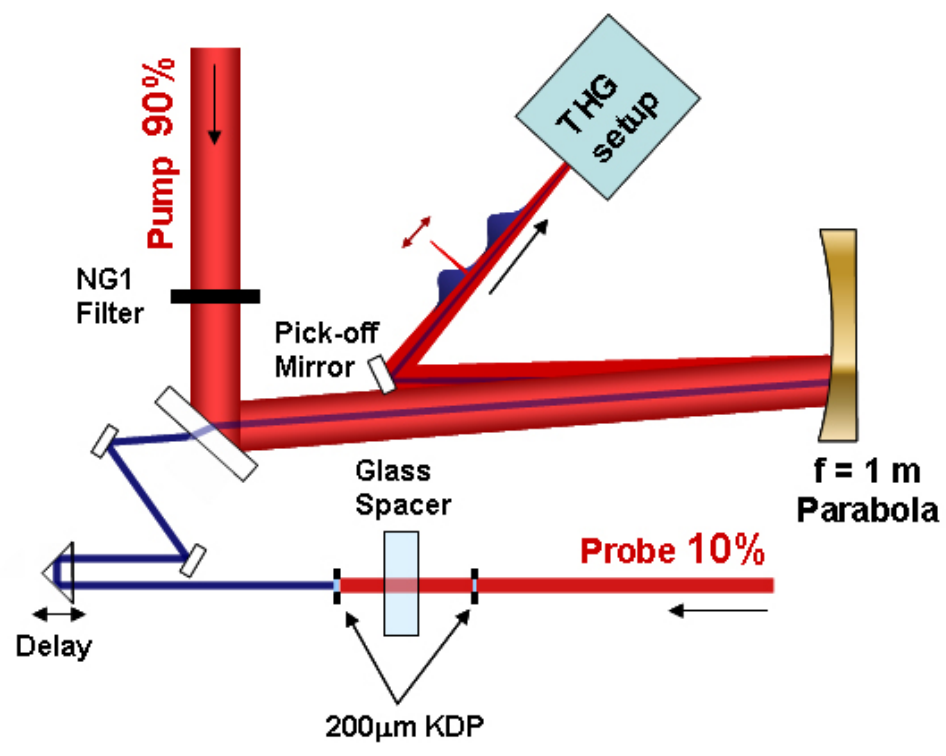

Figure 2.9: caption here

$I_{2 \omega}(t)$.

In this setup, the pump and probe beams are picked off after the parabola, but before the focus, to increase the intensity of the second harmonic. An NG1 filter must be placed in the pump beam, attenuating it by three orders of magnitude, in order to eliminate the contribution to the $3 \omega$ signal that the strong pump would generate on its own, which would swamp the third harmonic from the mixing of the pump and the probe. After the BBO crystal, a prism is used to separate the third harmonic from the first and the second, which are then blocked. A mirror picks off the third harmonic and sends it into a photomultiplier tube, and a UG11 filter eliminates scattered $800 \mathrm{~nm}$ and $400 \mathrm{~nm}$ light and other background sources. The resulting signal from the PMT is sent to an oscilloscope and averaged over many shots to improve the stability.

From the cross-correlation trace, we can estimate that the probe pulse has a duration of approximately $\tau_{p r} \sim 1.2 \mathrm{ps}$, the reference has a duration of $\tau_{r e f} \sim 0.6$ 


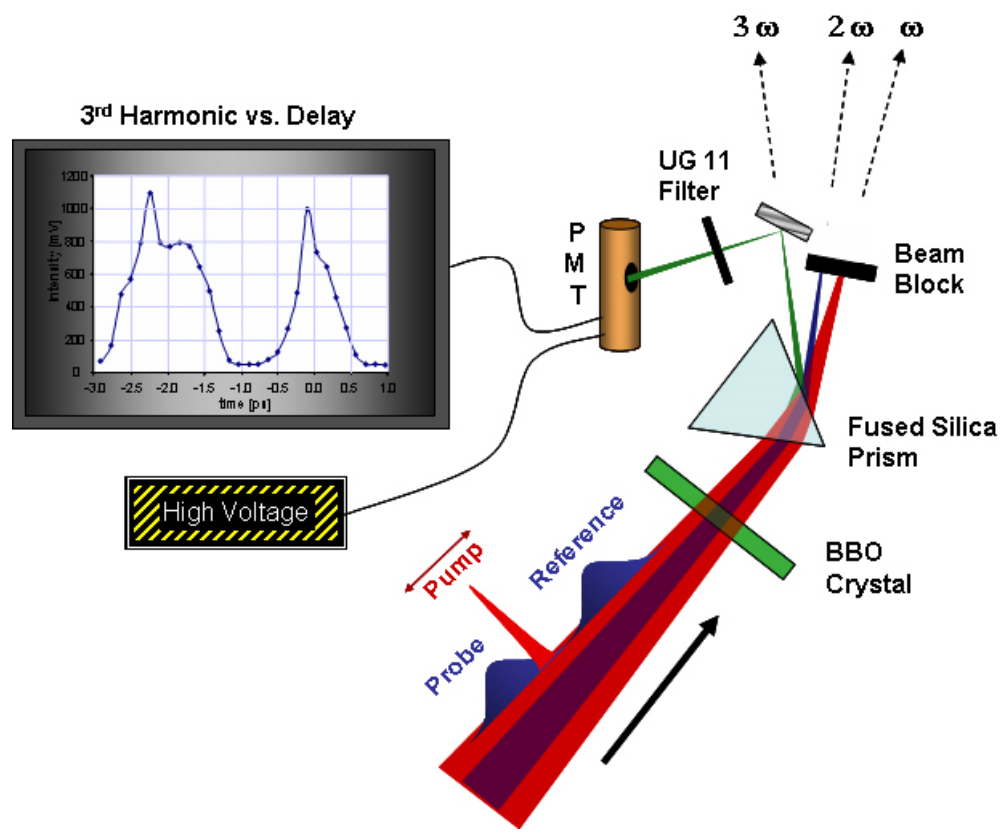

Figure 2.10: caption here

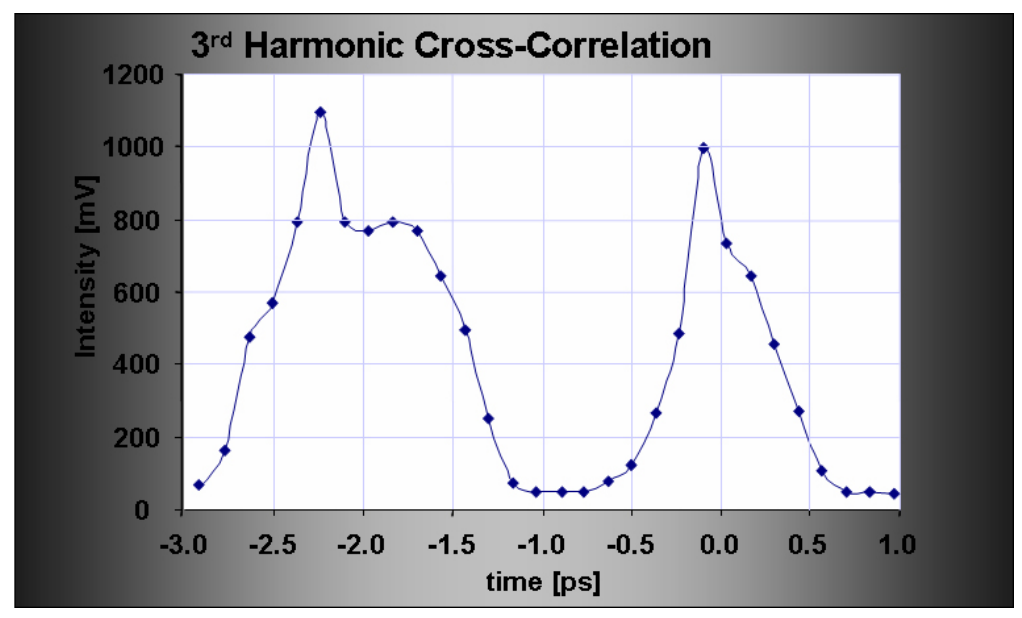

Figure 2.11: caption here 
$\mathrm{ps}$, and the pulse separation is $\Delta t_{\text {Group Delay }} \sim 2.1 \mathrm{ps}$. These figures compare closely to the rough predictions of $0.9 \mathrm{ps}, 0.7 \mathrm{ps}$ and $2.4 \mathrm{ps}$, respectively, made using linear dispersion theory, and are also adequately close to the design specs.

\subsection{Temporal Overlap}

One of the first milestones in any pump-probe experiment is the achievement of temporal overlap between the pump and probe pulses. This procedure necessarily begins with a crude method that brings the relative timing of the pulses close to zero, and then follows with more sophisticated and sensitive methods to refine the timing and to verify that the desired temporal alignment of the pump and probe pulses is achieved under the final experimental conditions.

\subsubsection{Crude Overlap}

The crude method of overlap is simply to measure the relative optical paths of the pump and probe lines from the point at which the pulses are separated to the point they are recombined. In the HERCULES laser system, there already existed a "transverse" probe line synchronized to the pump but intersecting it transversely at the interaction point for use in a shearing interferometer to measure the density profile of the gas coming from the gas jet. This probe line, which was picked off before the compressor and independently compressed was used as the fundamental for generating the second harmonic "longitudinal" probes. These second harmonic probes were then separated from the fundamental and recombined collinearly with the pump beam through the final High-Reflector in the pump line (figure 2.12).

The longitudinal probe pulse is then synchronized with the pump pulse by comparing the optical paths of the longitudinal and transverse probe lines from the point of separation to the gas jet and adjusting the probe delay leg. Accounting of contributions to the optical path was done meticulously as is shown in figure 2.13. 


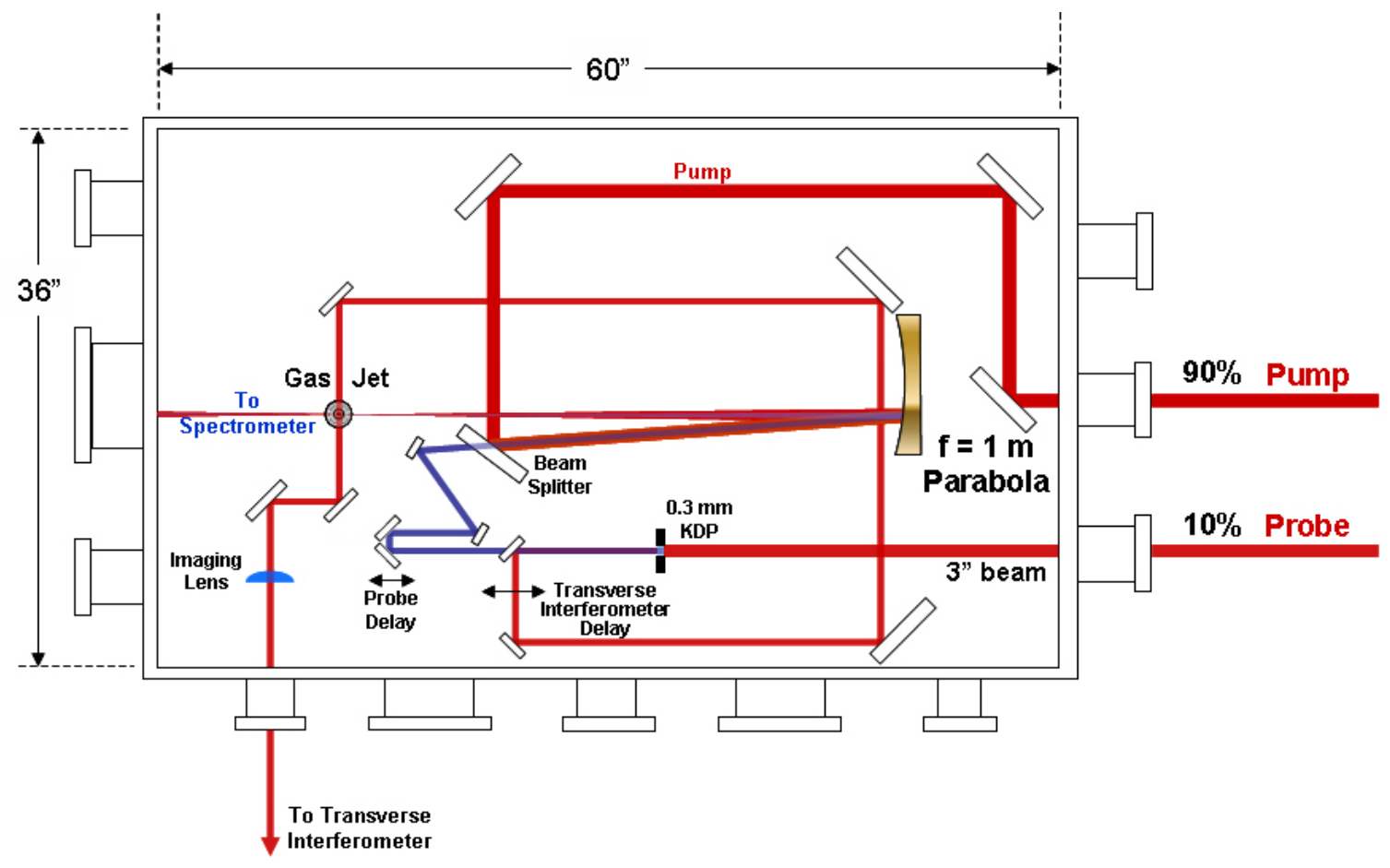

Figure 2.12: caption here 


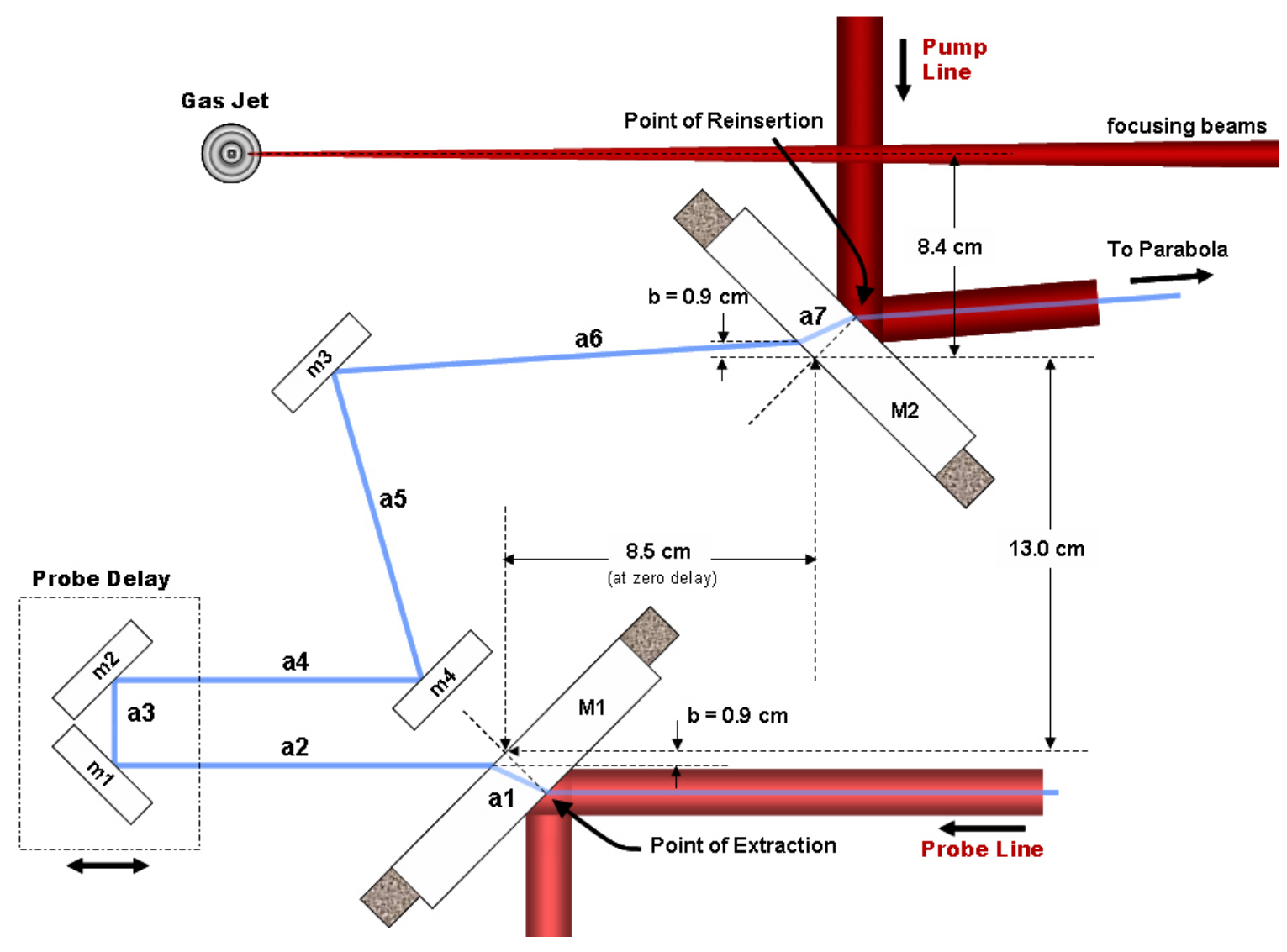

Figure 2.13: caption here

By this method, the relative timing of the pump and probe can be set to within a few picoseconds.

\subsubsection{Spatial Interferometry}

Once the pump and probe pulses are within a few picoseconds of each other, a timesensitive diagnostic is needed to achieve temporal overlap. Spatial Interferometry provides an ideal method for precisely overlapping pulses in time, since spatial interference fringes only appear when there is at least partial temporal overlap overlap of the pulses. A spare thin KDP crystal was used to up-convert a portion of the pump 


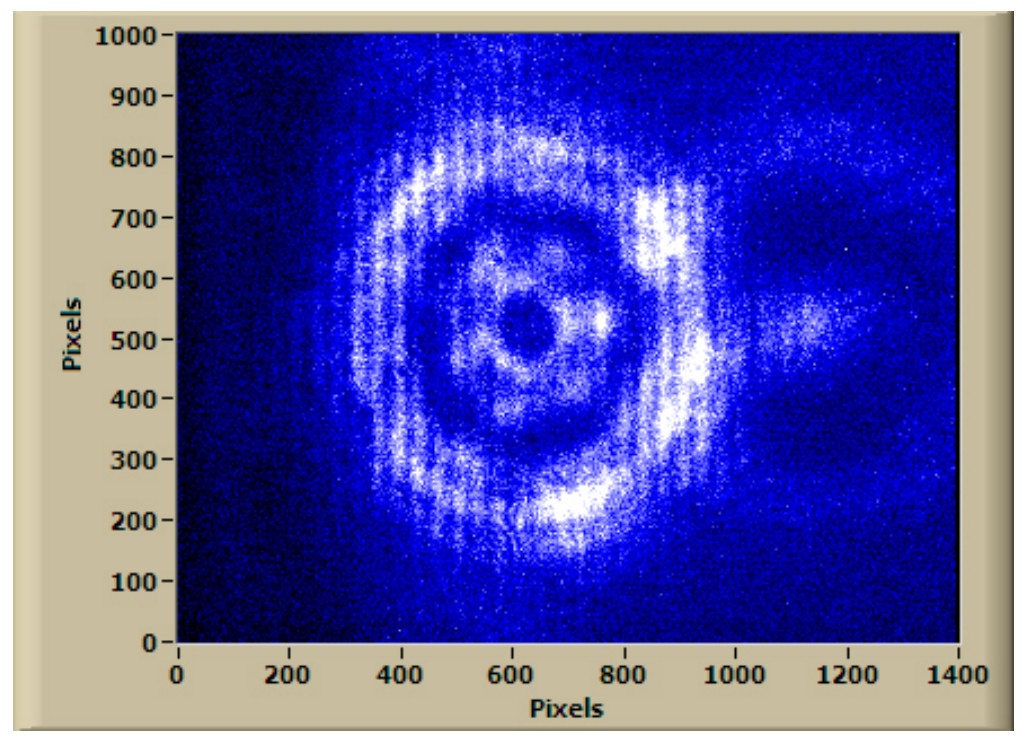

Figure 2.14: caption here

pulse to interfere with the probe. The crystal was placed in the beam line after the recombining beam-splitter and then angle-detuned to reduce the up-conversion efficiency, to allow matching the $2 \omega$ intensity with that of the probe, and the remaining pump fundamental was absorbed in a BG 39 glass filter. By slightly detuning the relative horizontal angles of the pump and probe beams, vertical fringes were produced (fig. 2.14) The offset Airy patterns from both beams is clearly visible. Because this measurement was done in alignment mode, the second harmonic intensity of the probe is very low, and the signal level of these spatial interferograms was just above the noise level of the CoolSNAP camera used to measure them.

The fringe contrast is directly related to the relative intensities of the two pulses in the volume of overlap. Fringe contrast can thus be used as a diagnostic for the degree of overlap between the pump and the probe pulses. In essence, spatial interferometry can be used to perform a cross-correlation measurement of the intensity profile of the probe pulse train. To evaluate the fringe contrast, a 1D 


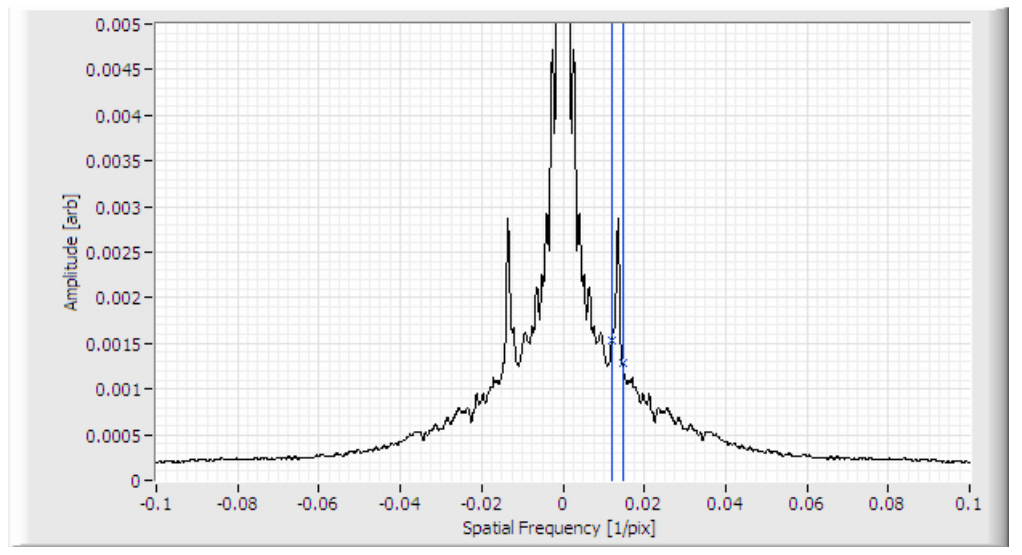

Figure 2.15: caption here

Fourier Transform is taken of the measured interferogram, and the rows are averaged to improve the signal-to-noise (fig. 2.15). The magnitude of the principal side-peak in the Fourier Transform is then recorded as a function of the relative delay between the pump and the probe pulse train. The result is shown in figure 2.16.

Although for the purposes of temporal overlap, it would suffice simply to detect and optimize the fringes between the pump and the probe, the extra step of mapping out the intensity profile of the two probes provides an additional confidence in the result of the procedure. Because interferometry invariably provides information only about the phase difference between two pulses, this technique only works if the reference pulse has no appreciable spectral phase. For example, if the pump and probe pulses were both chirped, then the interferometric "cross-correlation" would only provide a measure of the coherence between the two pulses, and so the result would be a temporal profile that was artificially short, and would not reflect the temporal extent of the probe pulse. In this case, the pump pulse is transform limited, so the interferometry provides a reasonable description of the pulse temporal profile. To demonstrate the validity of this statement, the interferometric cross-correlation is compared to the $3^{\text {rd }}$ order cross-correlation (fig. 2.17). 


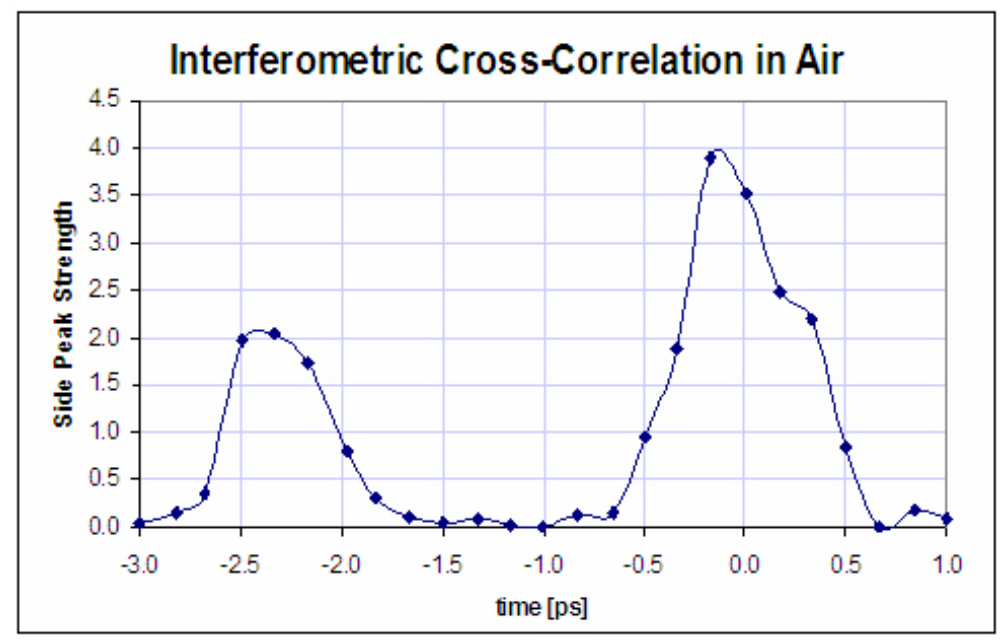

Figure 2.16: caption here

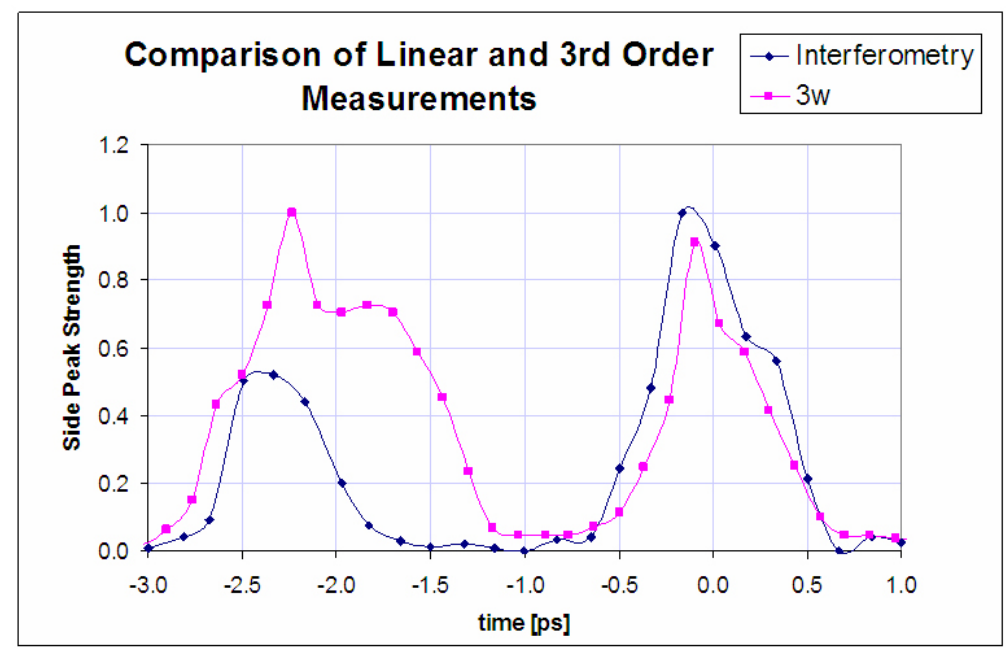

Figure 2.17: caption here 


\subsubsection{Ionization Front Signature}

Although in principal it should be possible to set up the Spectral Interferometry method with the experimental apparatus in its final (data taking) configuration, the measurements described above were done with the interaction chamber at air. Although it is not obvious, the process of evacuating the chamber can cause shifts in the temporal alignment of the pump and probe. In the experiments described here, a discrepancy of approximately 1.3 ps was observed between the temporal overlap condition with the chamber at air and at vacuum. This discrepancy is in part traceable to group-velocity dispersion between the $800 \mathrm{~nm}$ pump and the 400 $\mathrm{nm}$ probe in air over a relatively long path length (several meters). This discrepancy highlights the need for diagnostics that work in situ. Fortunately, the use of chirped probe pulses provides another method for diagnosing temporal overlap which is not only in situ and completely unambiguous, but also exceptionally easy to implement. This method works by observing the effect of an ionization front on the spectrum of the probe. In general, the extended duration (about a picosecond) of the pulse makes scanning for overlap very easy compared to the same procedure for femtosecond pulses because of the decrease in sensitivity to temporal misalignment. In addition, the spectral modulation imposed on the probe by the presence of the ionization front has a characteristic signature, and is in general a strong effect that can easily be observed by monitoring the spectrum. Figure 2.18 shows a typical probe spectrum modulated by an ionization front. The pattern of modulation is easily explained: the ionization front represents a sudden jump in the degree of ionization of the gas; the sudden increase in electron density is accompanied by an decrease in the index of refraction, which results in a temporally-varying phase-shift with a steep gradient; this gradient induces a blue-shift of the local spectrum; the blue-shifted component of light can then interfere with light of a similar wavelength but a different temporal location, and induce spectral fringes as a result. In essence, the localized blue- 


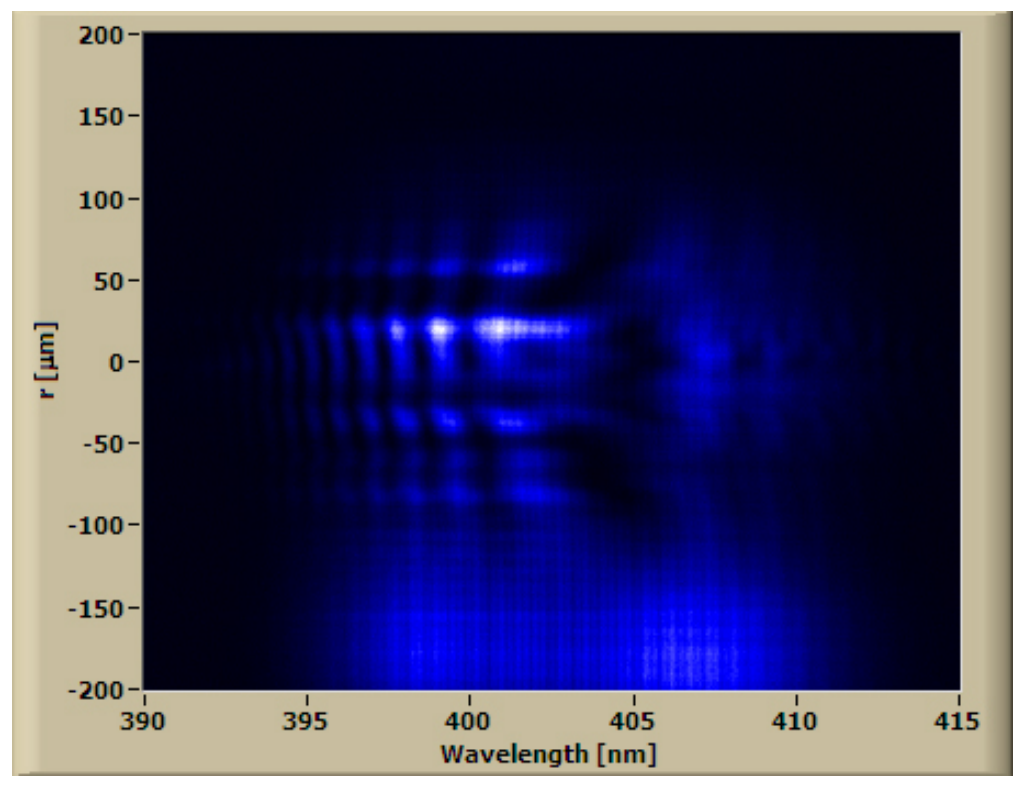

Figure 2.18: caption here

shifting of a chirped pulse causes it to self-interfere. In figure 2.19, the modulation is simulated by adding an abrupt temporal phase shift to the electric field of a chirped pulse, and compared to a lineout from the measured spectrum. The chirp, the phase amplitude and front-steepness and relative timing were adjusted to fit the data, but it is clear that the essential features are well reproduced by this simple model.

It is one of the factors that makes this technique so useful that the pattern of the modulation features is preserved as the delay between pump and probe is varied. The pattern simply shifts with respect to the spectral amplitude profile as the relative delay is changed as a result of the time-wavelength correspondence that comprises the chirp (fig 2.20). Roughly speaking, the ionization front overlaps in time with the probe wavelength corresponding to the deepest dip in the spectrum. Thus it is relatively trivial to temporally align the pump and probe to the desired configuration simply by adjusting the position of the modulation pattern with re- 


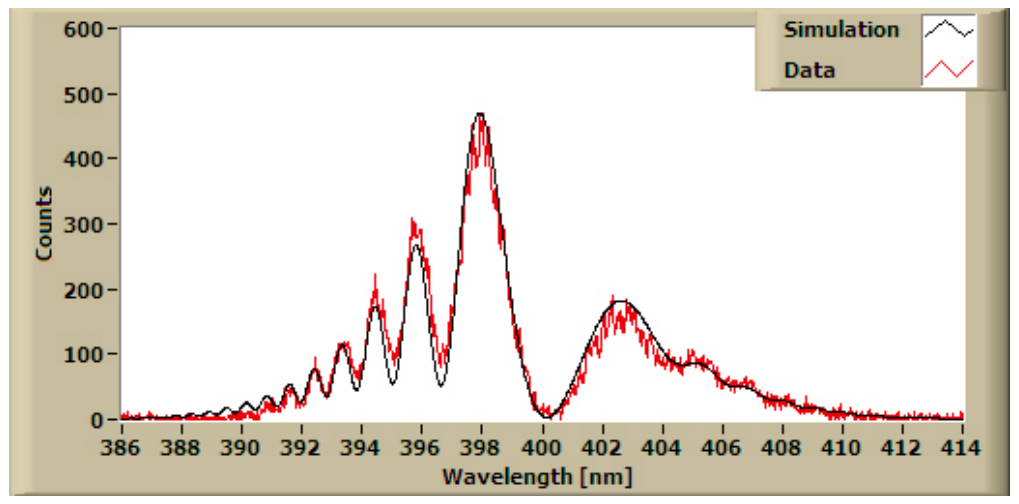

Figure 2.19: caption here

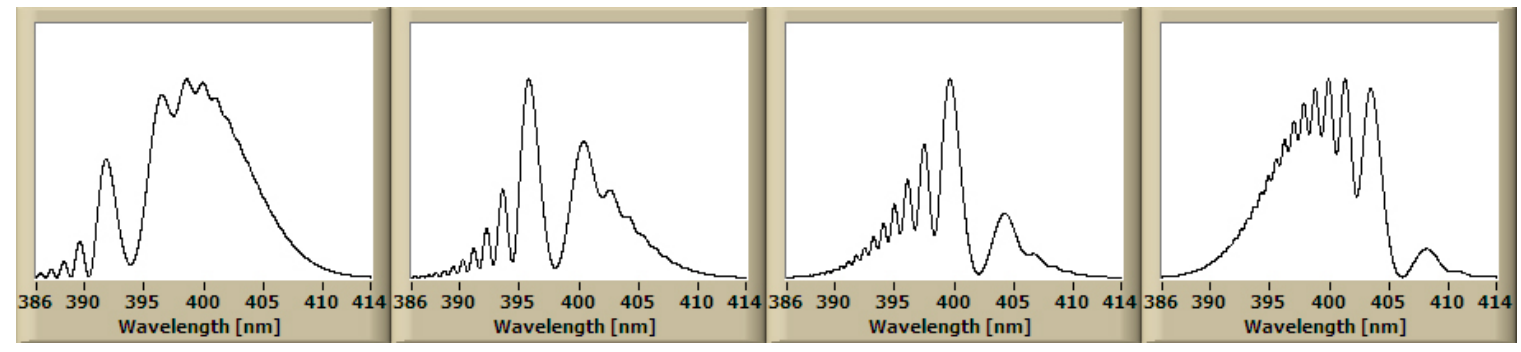

Figure 2.20: caption here

spect to the peak of the probe spectrum. Because this technique can be done with the laser system in the final configuration for data taking, there is no danger of misadjusting the timing between the alignment phase and the data-taking phase of the experiment.

\section{$2.3 \quad$ Spatial Overlap}

After temporal alignment has been accomplished, the next most important procedure is the spatial overlap of the pump and probe. This is accompanied with the need to align the probe imaging system so that the pump-affected region of the probe will be imaged (or mapped) onto the entrance slit of the spectrometer. These 
two procedures are coupled, and can be understood with the following analogy of taking a conventional photograph. There is an object, which is the wakefield; there is a flash bulb, which is the probe pulse, that illuminates the object; there is a detection medium (film), which is the CCD of the spectrometer; and there is an imaging system with an aperture (the spectrometer entrance slit).

The two procedures mentioned above can be understood in terms of this analogy. Spatially overlapping the pump and probe is like directing the light from the flash onto the object. But unlike the case of conventional photography, the illumination photons come at a premium, so the luxury of sending illumination light everywhere is not feasible. Instead, the probe light is concentrated on the object (by focusing). The region of illumination is made to be somewhat larger than the object in order to be able to see the background. The second procedure, of aligning the imaging system, is analogous to pointing the camera at the object.

\subsubsection{Overlapping the Pump and Probe}

As with the temporal alignment procedure, the spatial overlap takes place in multiple steps. After the first step of crude alignment by eye, the first level of refinement can be made with a camera observing interference between the probe and the pump in the second harmonic as was done in the temporal alignment section (see fig. 2.14). The spatial interference offers a powerful method of alignment, because at a single plane of observation, both the relative position of the two beams (intensity profiles) and the relative angle between them (interference pattern) can be measured. When both modes are overlapped, and the interference fringes are larger than the image, (fig. 2.21), then both beams are taking nearly the same path. This alignment is then refined by observing the focused modes of the two beams in the spectrometer set to zero-order. "Zero-order" simply means that the grating is adjusted for zerowavelength, and thus acts simply as a mirror, without spectrally dispersing the 


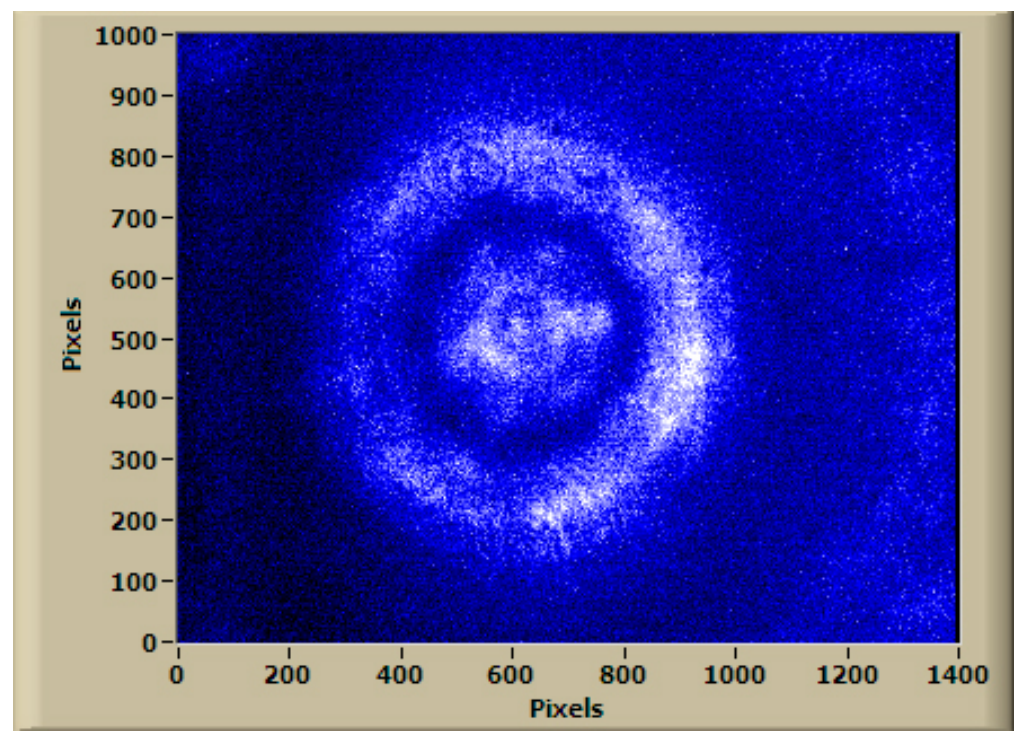

Figure 2.21: caption here

beam. The entrance slit is also opened to its widest extent to allow observation of both spatial dimensions.

Although this method is very sensitive, the alignment achieved by it can not be trusted as a final alignment because of the necessity of inserting an optical element (i.e. the KDP crystal) into the beam line to up-convert the pump. Even a thin well-polished crystal can not only deflect both beams, but can induce a small relative angle between them. To avoid this issue, one might consider trying to overlap the image of the probe with the image of the pump at its fundamental. However, because the pump and probe are of different wavelengths, chromatic effects in the lens-based imaging system can cause two non-overlapped beams of different colors to appear to be overlapped in the image plane. Although this effect can be mitigated if the lenses are aligned perfectly, it is usually unwise to rely on this. 


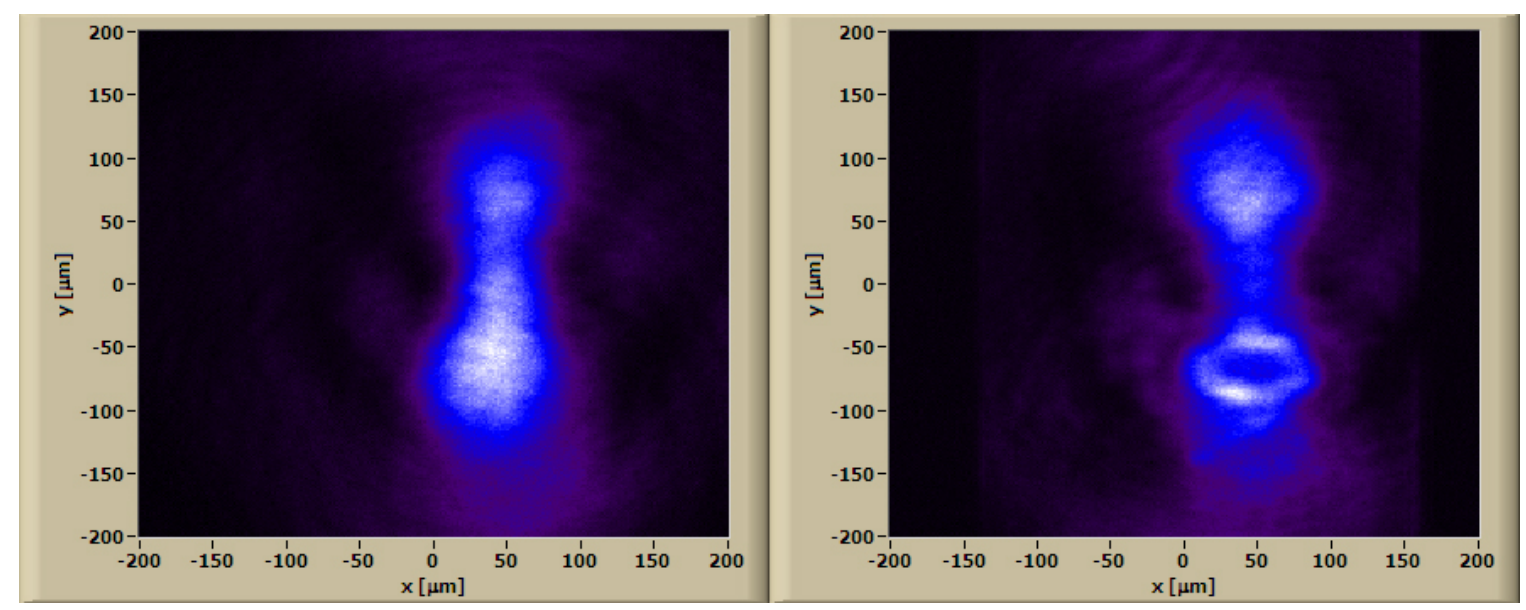

Figure 2.22: Defocusing Diagnostic

\subsubsection{Ionization-induced Defocusing}

Both the two-color method and the one employing overlap at the second harmonic can serve to get the alignment very close, but the necessity of precision in the final alignment dictates a method that is both direct and in situ, such as the ionizationfront diagnostic used in the temporal alignment. Fortunately, such a method exits. The ionization column produced by the pump can act as a defocusing lens to the co-propagating probe beam, and the signature of this defocusing can be used as a diagnostic of spatial overlap of the probe and the ionization column, and thus, of the wakefield. In figure 2.22 , the left image shows the imaged probe mode in the absence of gas, and the right image shows the effect of the ionization-induced defocusing on the probe with the gas jet backed by 10 psig.

The effect of mis-aligning the illumination of the target can be seen clearly in figure 2.23. Here the location of the target (i.e. the plasma and wakefield) is fixed and the alignment of the probe is being adjusted to center it on the object.

The second half of the alignment procedure is to overlap the image of the wakefield with the slit of the spectrometer. This process is depicted diagramatically 

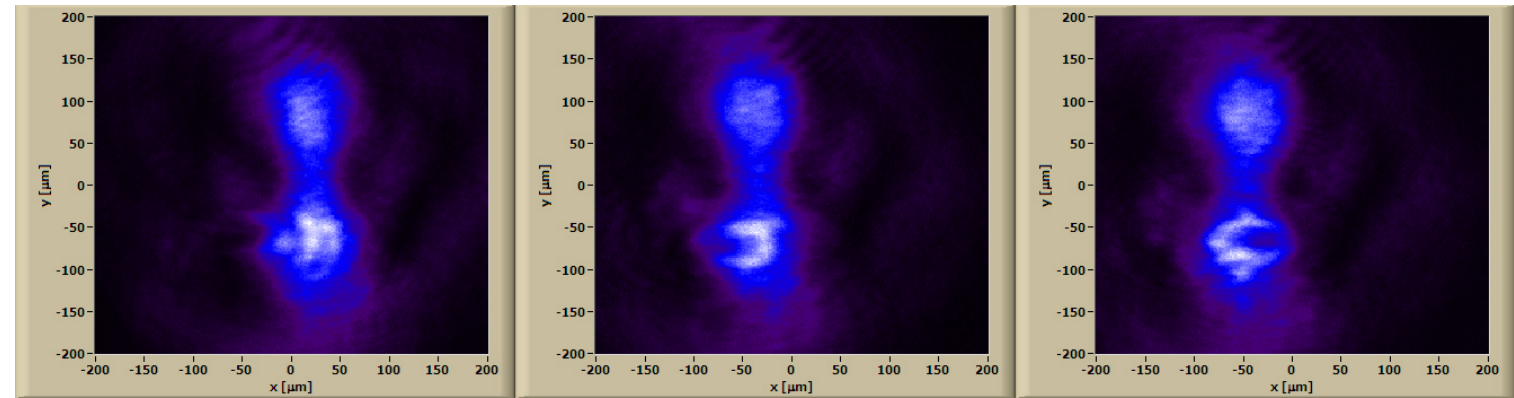

Figure 2.23: Defocusing Diagnostic

in figure 2.24.

Now that the location of the plasma can be correlated to the region of defocusing, it is straight-forward to align the defocusing spot onto the slit. By partially closing the slit, the relative position of the target and the slit can be seen (fig. 2.25).

An important detail of this alignment procedure is that in order to see the probe mode and the defocused spot, it is necessary to block light from the pump which has been blue-shifted by the ionization and wake-generation process. This "continuum" generation can be quite strong, and can at high enough densities span the full octave from $800 \mathrm{~nm}$ down to $400 \mathrm{~nm}$, interfering (figuratively) with the measurement process and (literally) with the probe light (fig 2.26). At the lower densities used for the overlap test, the continuum light does not reach down to $400 \mathrm{~nm}$, but it is still within the acceptance bandwidth of the optics and filters in the probe imaging line. Since the spectrometer is working in a non-dispersive mode for this diagnostic, the continuum light easily reaches the detector and swamps the image of the probe. To avoid this, an interference filter with a narrow bandwidth centered at the probe wavelength is inserted (for the duration of the measurement) between the spectrometer and the CCD camera at the detection plane. This placement is important, because if the filter were placed before the entrance slit, refraction by the filter could introduce errors in the relative alignment of the wakefield image and the 


\section{Pump - Spectrometer Slit Overlap}
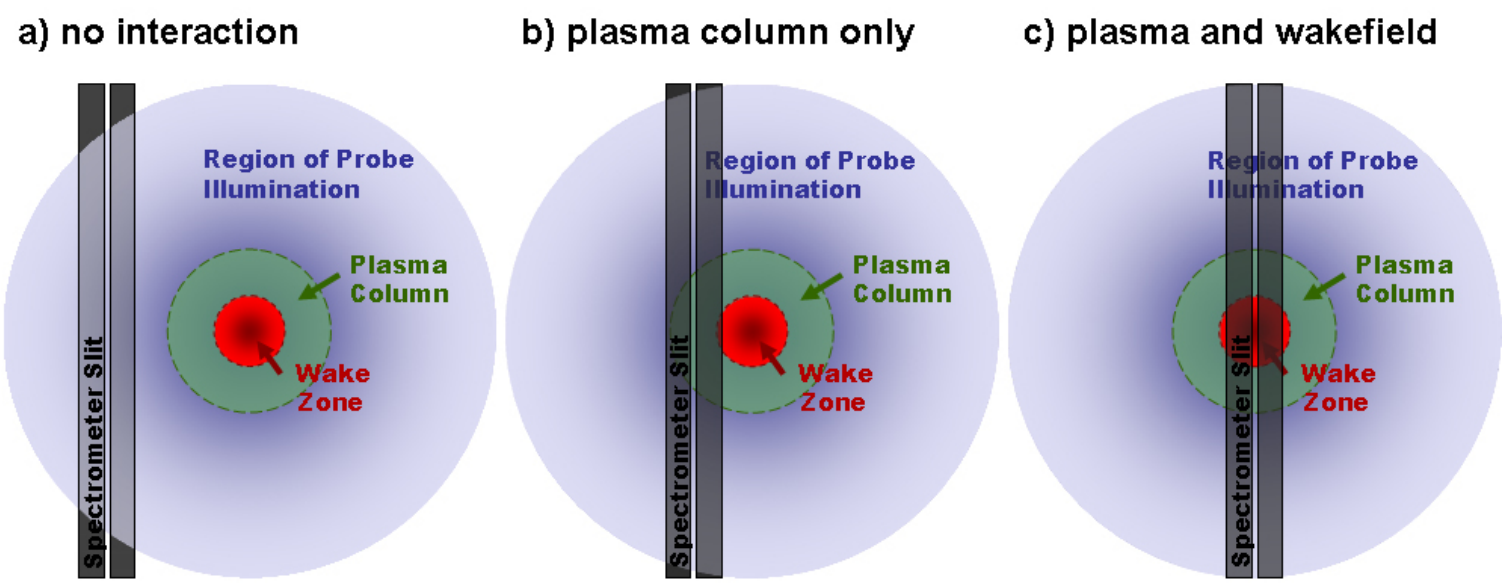

Figure 2.24: Relative Alignment of Pump and Cross-section of Measurement
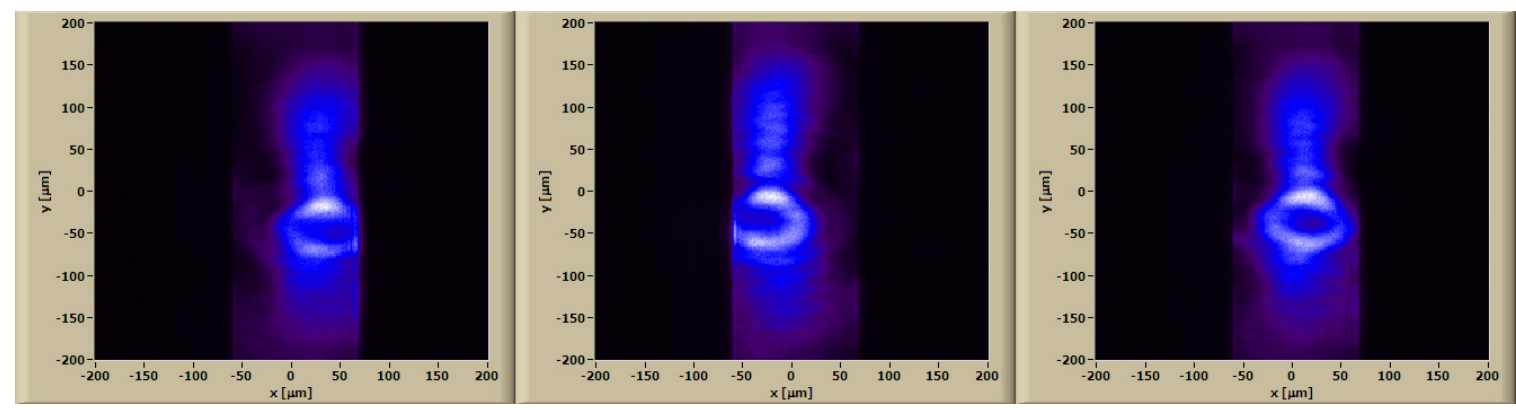

Figure 2.25: Image of the defocused probe mode, shadowed by the slit 


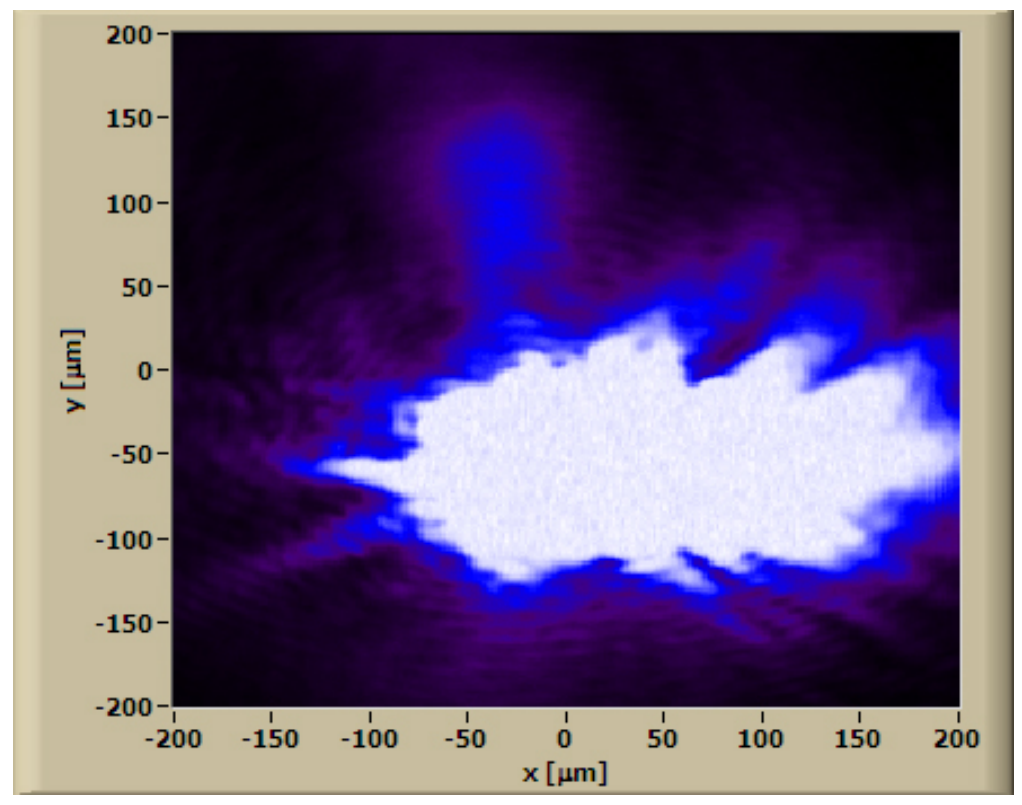

Figure 2.26: Super-continuum light generated by the interaction of the pump with the plasma

spectrometer slit. By inserting the filter after the slit, this ambiguity is removed, and the imaging of the slit onto the camera is not excessively impaired.

\subsection{Density Determination}

The density profile of gas jet was measured with a rooftop shearing interferometer (fig. 2.27. A single beam comprising the remaining fundamental from the FDH probe line intersects the interaction region synchronously with the pump beam, but orthogonal to it. The beam then imaged to the interferometer where a beamsplitter splits the input beam 50-50, sending one beam to a retro-reflecting mirror, and the other to a rooftop prism which inverts the beam vertically. The two beams are recombined with the same beam-splitter, and interfered on a CoolSNAP CCD detector. The interferometer thus works by using half of the beam as a reference 


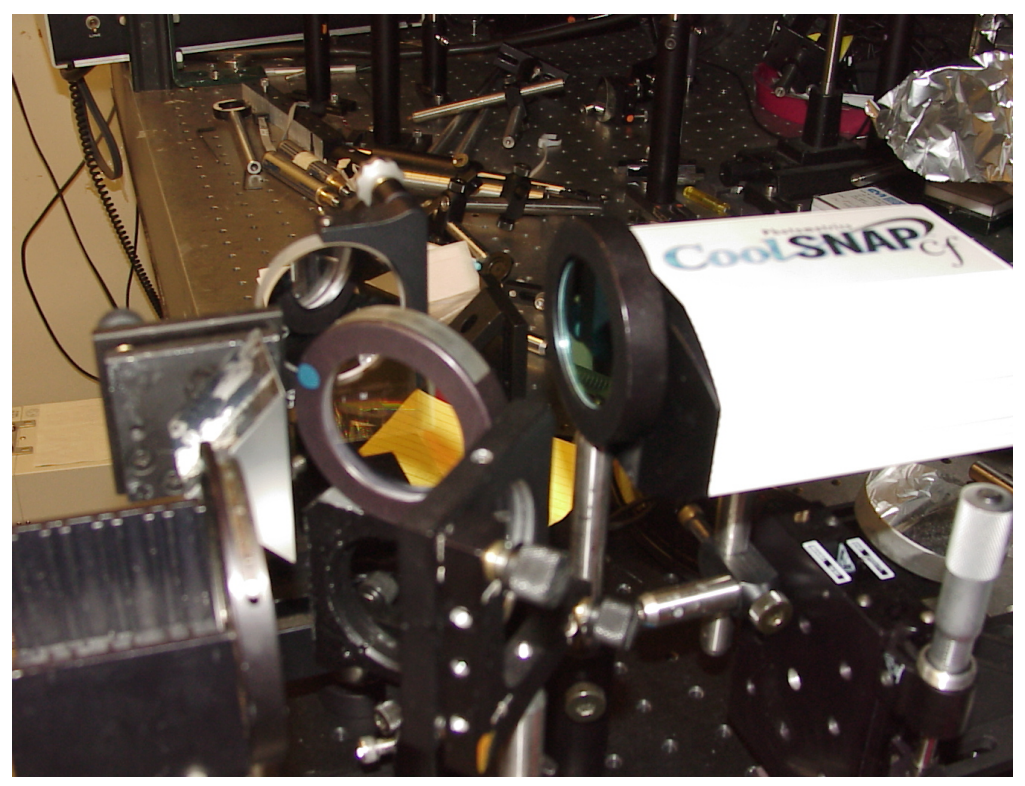

Figure 2.27: Transverse shearing interferometer

for the other half, avoiding the necessity of splitting the beam inside the chamber, and making the device considerably more stable and compact than a traditional Mach-Zehnder interferometer. In addition, because the interferometer is not inside the chamber, its alignment doesn't suffer the deleterious effects of cycling between air and vacuum.

\subsubsection{Phase Retrieval}

The shearing interferometer works by spatially interfering one beam, which experiences spatially-varying phase shifts from an interaction, with another beam which avoids the interaction and acts as a reference. A small horizontal angle between the two beams introduces a vertical interference fringe-pattern to the intensity profile recorded on a CCD where the beams intersect (fig. 2.28).

The transverse location of the fringe maxima on the CCD is sensitive to the relative (spatially-varying) optical phase between the two beams. In order to spa- 


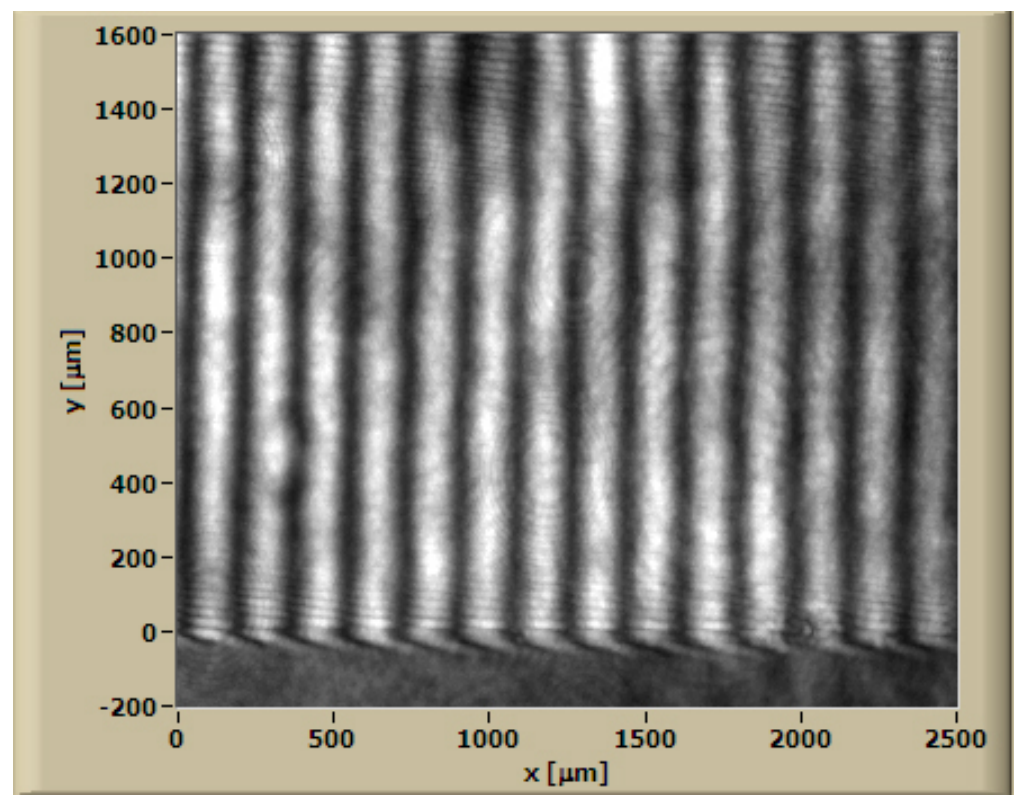

Figure 2.28: Shearing Interferogram with no gas

tially resolve these phase-shifts, the object-plane at the interaction must be imaged onto the camera. This is done with a singlet lens providing a magnification of approximately $\mathrm{M} \cong 2.6$. Figure 2.29 shows an image of a mesh with $50 \mu \mathrm{m}$ spacing located at the object plane. Calibration of the image is done my measuring the the inner diameter of the mesh mounting ring (ID $2.29 \mathrm{~mm}$ ) with a Vernier caliper. The resulting calibration was $1.800 \mu \mathrm{m} /$ pixel.

To measure the density of the plasma at the interaction, the transverse probe must traverse the interaction region shortly after the formation of the plasma filament by the driving laser pulse. If the delay between the driving pulse and the probing pulse is too long ( $\gtrsim$ nanosecond), ion dynamics come into play, and there will be a small modification of the plasma density profile. To find the timing corresponding to temporal overlap, the delay-arm of the transverse probing pulse is adjusted until the ionization front is visible in the interferometer image. Figure ?? shows a shadow-graph of the plasma filament produced by blocking one arm of the 


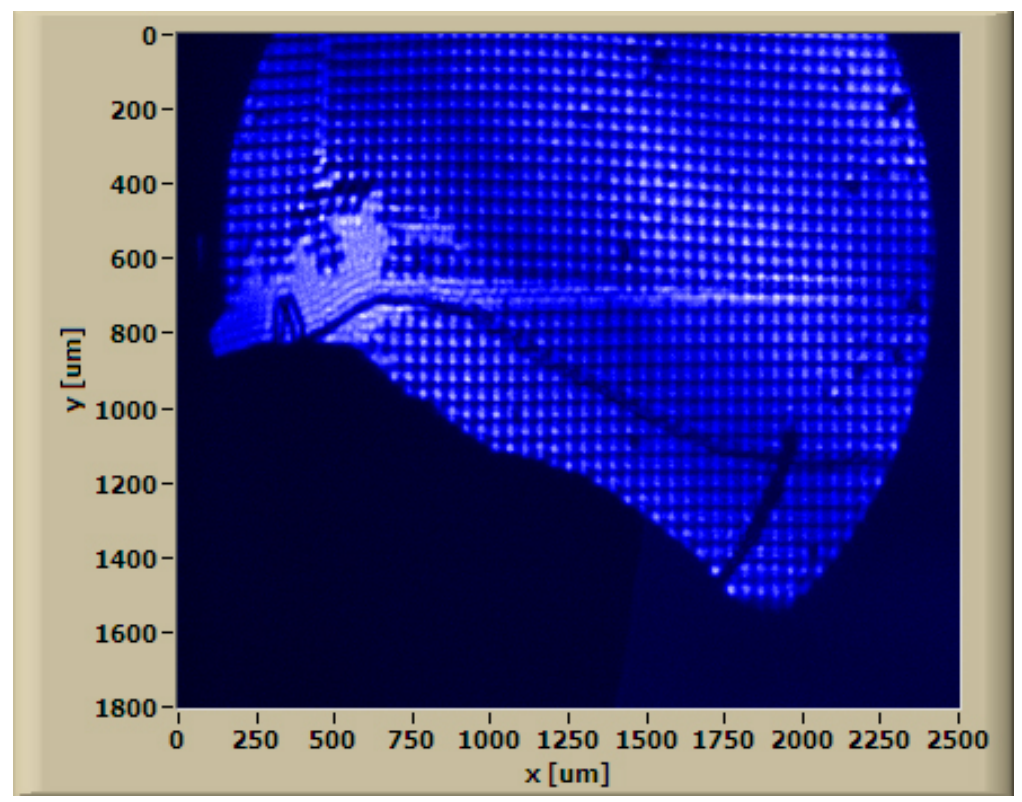

Figure 2.29: Image of $50 \mu \mathrm{m}$ mesh using Transverse Interferometer

interferometer. The abrupt edge to the filament on the right side is evidence that the probing pulse caught the pump pulse in the act of traversing the field of view from left to right, generating a plasma column behind it.

Although the probe beam passes through both gas and plasma, only the plasma contributes significantly to the phase of the probe. Figure 2.31 shows an interferogram demonstrating a phase shift from a plasma column. The driving pulse has already passed beyond the field of view of the image. The phase shift is localized to the region occupied by the plasma, and the degree of shift (as indicated by the bending of the fringes) is determined by the difference in optical path-length traveling through the plasma compared to that traveling through the neutral gas. The phase shift is thus an integrated effect dependent on both the plasma density and on the thickness of plasma traversed. In general, when the plasma density is changing along the path of the probe, the phase shift is given by: 


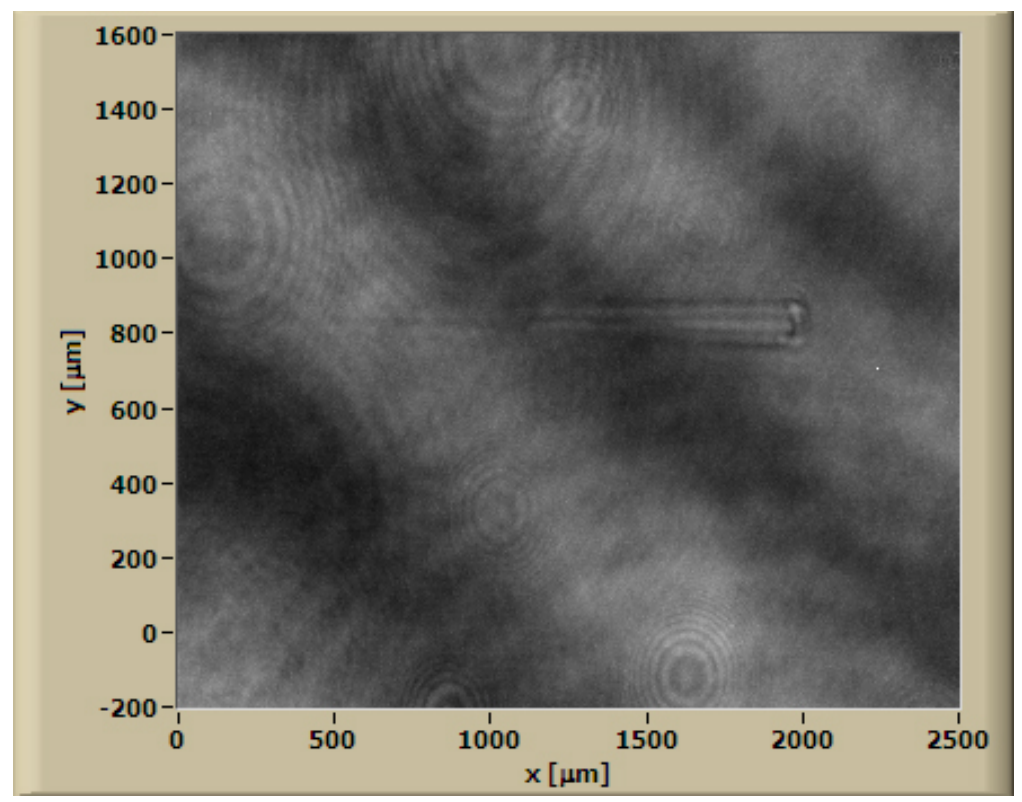

Figure 2.30: Shadow-graph of plasma column and ionization front

$$
\Delta \phi=\int_{-\infty}^{+\infty} \frac{2 \pi \eta\left(n_{e}, z\right)}{\lambda} d z
$$

where $\eta\left(n_{e}, z\right)$ is the local index of refraction, dependent on the electron density as:

$$
\eta\left(n_{e}\right)=\sqrt{1-\frac{\omega_{p}^{2}\left(n_{e}\right)}{\omega^{2}}}
$$

where $\omega_{p}$ is the plasma frequency, given by:

(** equation for plasma frequency $* *$ )

The procedure for recovering the phase shift procedes by.... (**??**) (see fig. 2.32) 


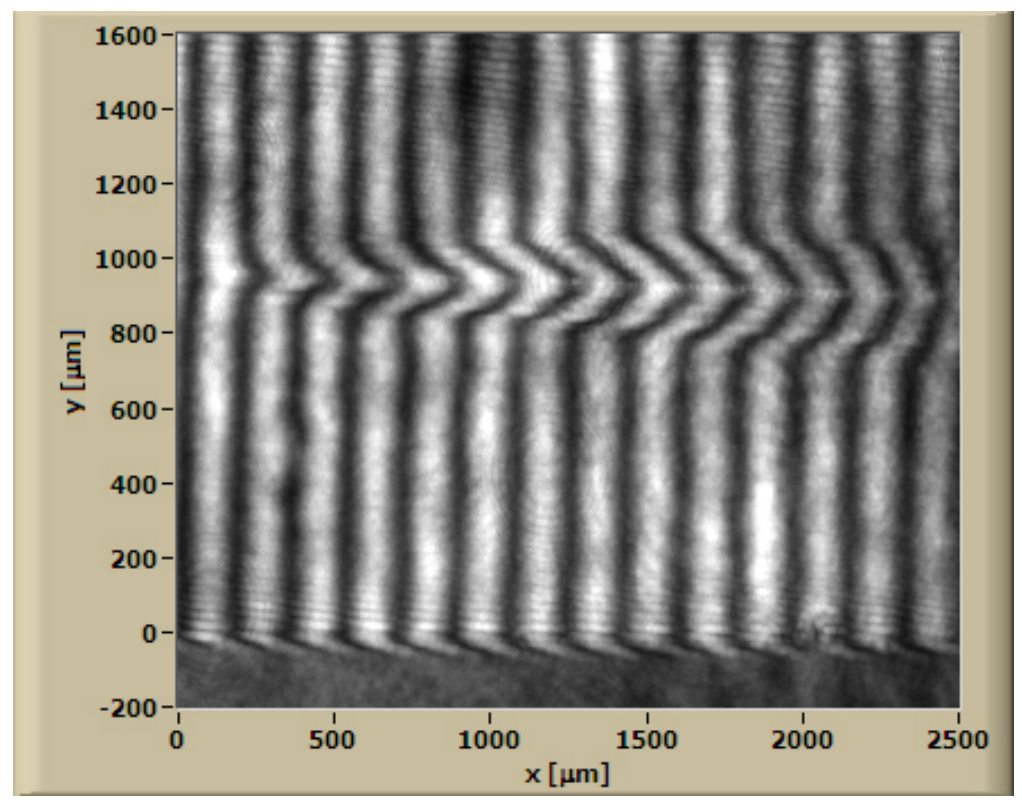

Figure 2.31: Shearing Interferogram with plasma filament

\subsubsection{Abel's Inversion}

In general, it is not possible to determine the electron density profile $n_{e}(z)$ simply by the measurement of $\Delta \phi$. However, if we make the assumption of cylindrical symmetry, (which while not in general true, is at least not unreasonable as an approximation), the spatial variation in the phase shift, $\Delta \phi(y)$ can be used to construct a radially symmetric electron density profile $n_{e}(r)$ using a process known as Abel's Inversion. An Abel Inversion is the process of inverting the equation

$$
S(y)=\int_{y}^{R} f(r) \sqrt{r^{2}-y^{2}} r d r
$$

to get the equation:

$$
f(r)=-\frac{1}{\pi} \int_{r}^{R} \frac{d S(y)}{d y} \sqrt{y^{2}-r^{2}} d y
$$



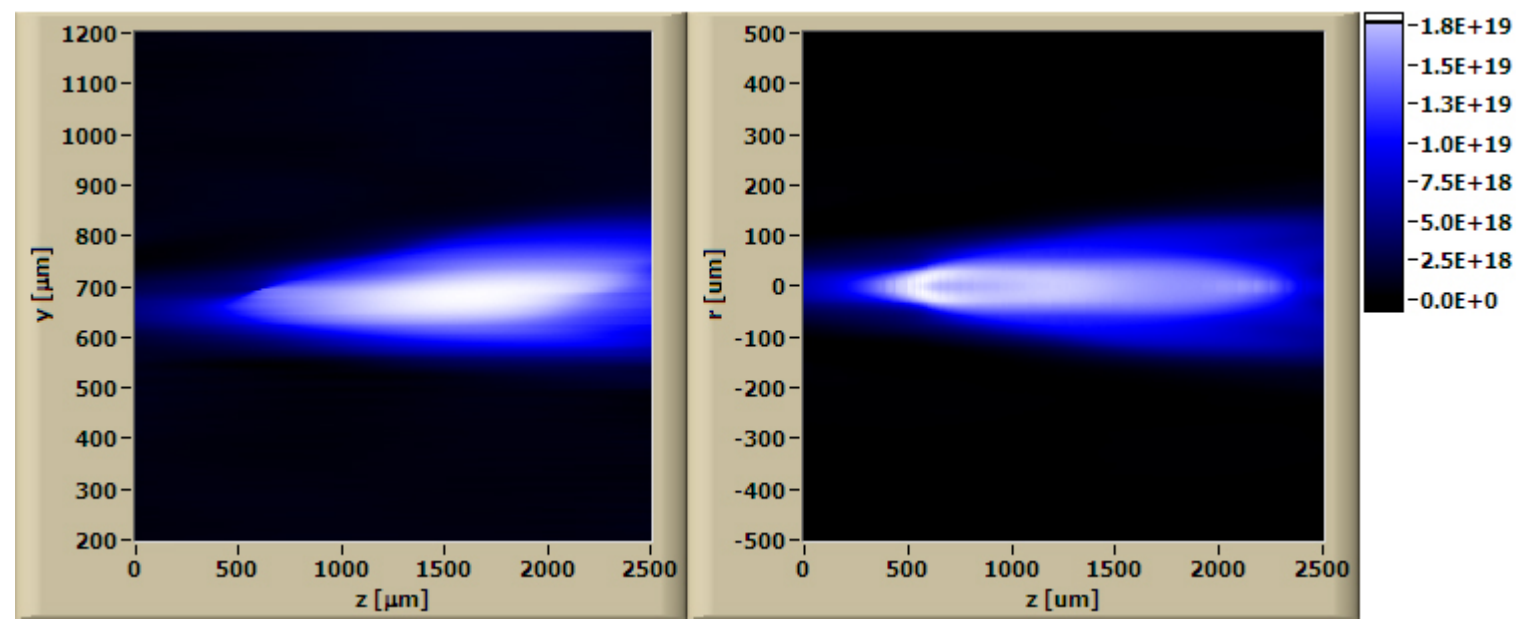

Figure 2.32: Abel Inversion

where $\mathrm{S}(\mathrm{y})$ is a measurement that results from integrating a quantity transversely through a cylindrical profile, and $\mathrm{f}(\mathrm{r})$ is the radial dependence of the desired quantity. Many numerical implementations of this inversion have been established. The analysis here directly employs a Fourier-Transform based approach as described in [5]. The essence of the technique is to expand $\mathrm{S}(\mathrm{y})$, (assumed to be symmetric about $\mathrm{y}=0$ and zero outside of the range $y \in\{-R, R\})$ in terms of symmetric Fourier basis functions, and to compute the Abel inversion of these functions. The Abel inversion of $\mathrm{S}(\mathrm{y})$ is then constructed as a sum of Abel-inverted functions that are standard for all data. This approach has the dual advantage of being fast, and of being relatively insensitive to the exact definition of symmetry point. The Abel inversion that is produced yields information about the longitudinal (along the direction of the driving pulse) electron density profile, as well as the longitudinal evolution of the driving beam spot size (fig. 2.32 and fig. 2.33). Together, these data can be used to ascertain the location of the pump focus with respect to the density profile. 


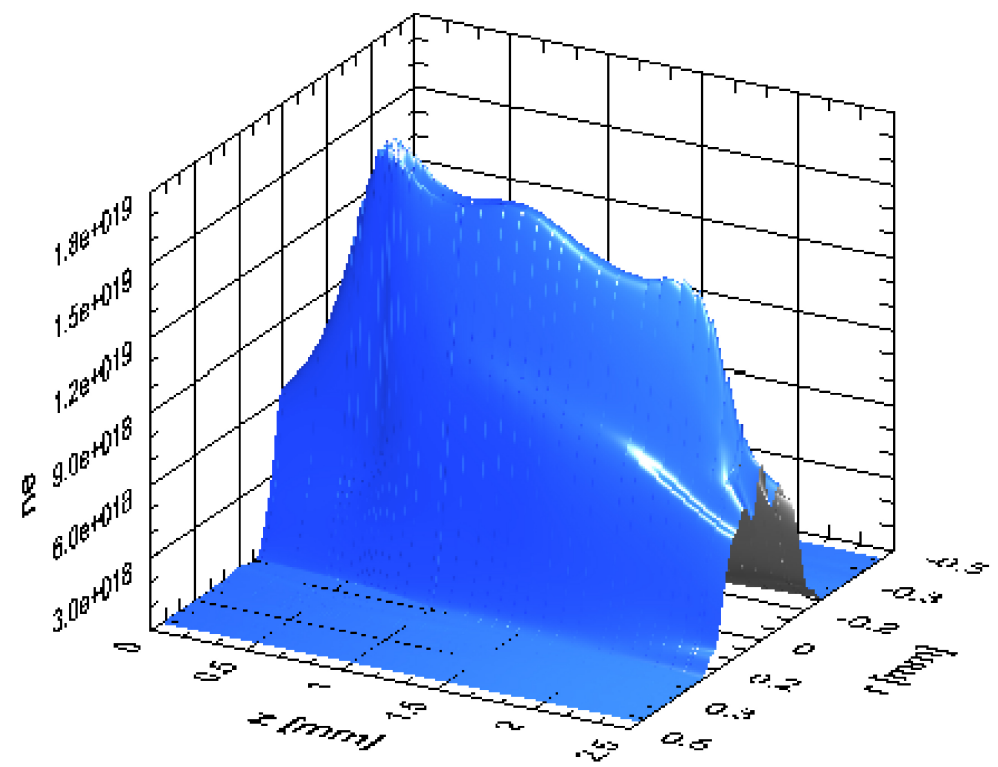

Figure 2.33: Abel Inversion

\subsubsection{Adjusting the Density}

\subsection{Probe Imaging Setup}

In order to resolve variations in the spatial structure of the wakefield, an imaging system was implemented (figure 2.36). Two plano-convex lenses of focal length 30 $\mathrm{cm}$ and $100 \mathrm{~cm}$ were used to image the exit plane of the plasma onto the spectrometer input plane (i.e. the entrance slit), with a magnification of approximately 3 .

\subsubsection{Choosing the Magnification}

This magnification was chosen to optimize the spatial resolution while still having enough probe intensity on the camera to yield a good signal-to-noise ratio, and having a large enough field of view to see the entire object, including some surrounding space to act as a null reference. The spectrometer images the slit onto a CCD 


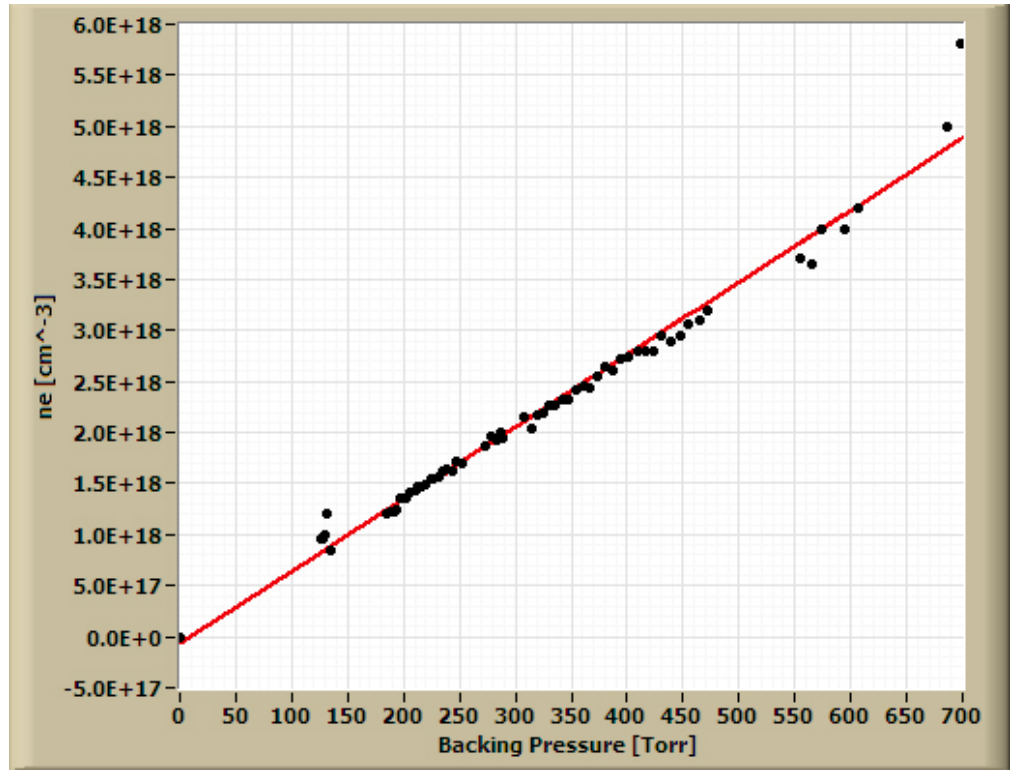

Figure 2.34: Peak density vs. jet backing pressure

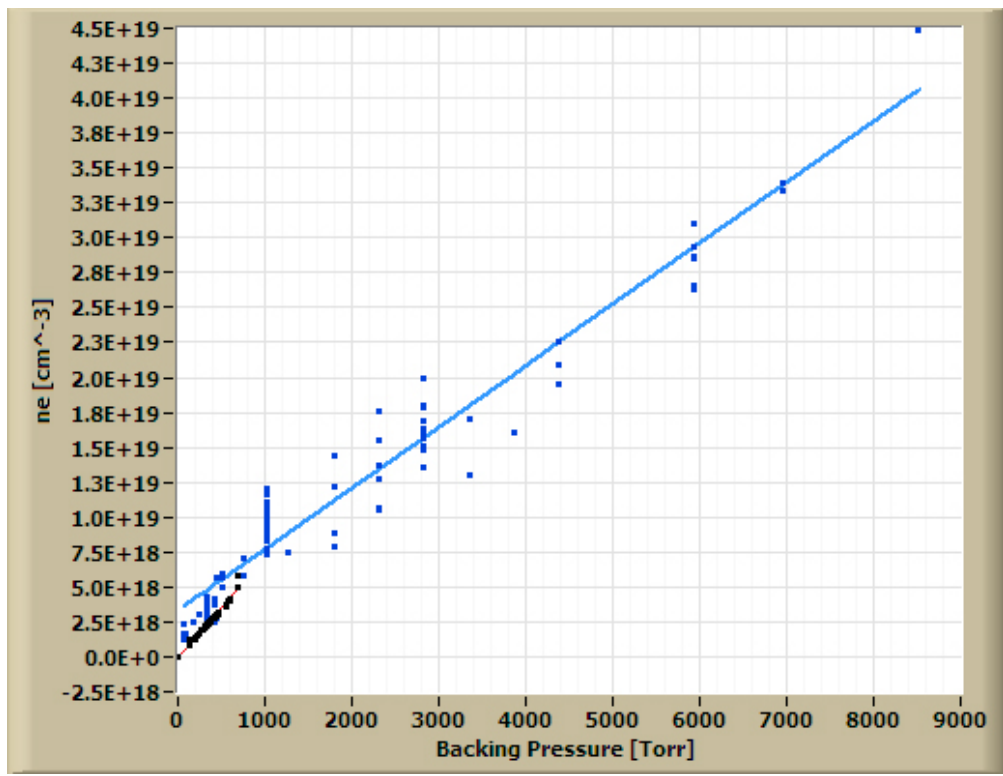

Figure 2.35: Peak density vs. jet backing pressure 


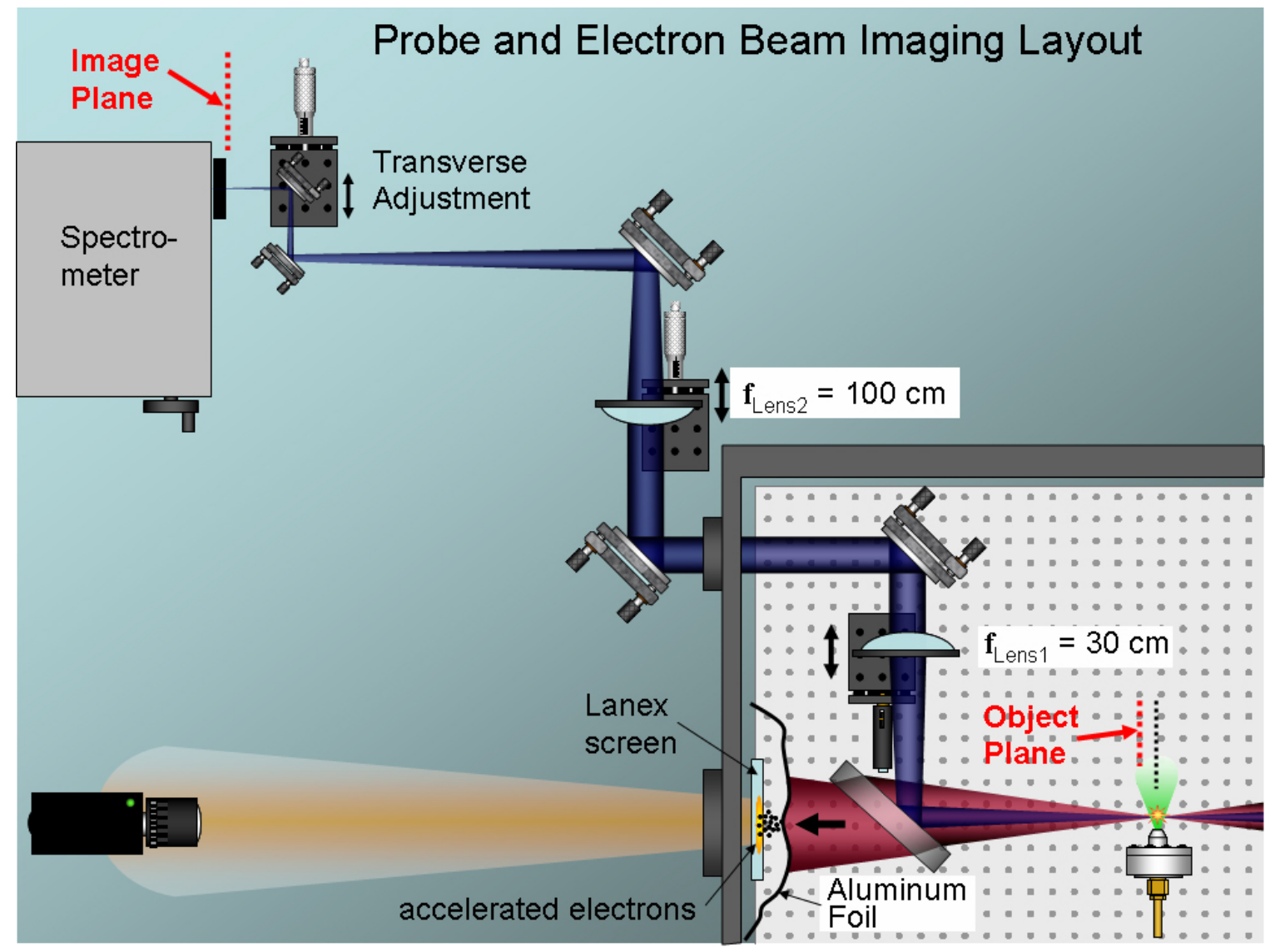

Figure 2.36: caption here 
camera with a 1:1 magnification in the vertical direction, while spectrally resolving the Fourier Plane in the horizontal direction. The CCD used for the spectrometer was a 12-bit, cooled CoolSNAP Cf camera from (**?**) with 1400 x 1040 pixels, and a pixel size of $4.65 \mu \mathrm{m}$. Accounting for magnification, the theoretical limit to the spatial resolution of the imaging system is then set by the extent of the object plane captured by a single pixel, which is approximately $1.4 \mu \mathrm{m}$. In practice, the actual resolution was limited by the $\mathrm{f} / \#$ of the optics and by the abberations of the optical system to a value somewhat larger than this, thus there would be no benefit to increasing the magnification beyond what was used.

\subsubsection{Calibrating the Spatial Dimension of the Spectrometer}

Determination of the spatial calibration of the imaging system was done by imaging a fine mesh mounted on a metal ring with a grid-spacing of $50 \mu \mathrm{m}$ onto the spectrometer CCD with the slit open and the grating set to zero-order (see figure 2.37). In this mode, the grating acts like a non-diffractive optic (mirror), and the spectrometer simply images the plane of the slit onto the CCD. The mesh was placed at the object plane, (near the focus of the pump), and second-harmonic light from the pump beam up-converted in a thick $(2 \mathrm{~mm}) \mathrm{LiIO}_{3}$ crystal was used to illuminate it. The pump beam was used as a light source instead of the probe because the measurement was done with the laser system at low power, and the probe did not provide sufficient intensity in this mode of operation. The pump beam was strongly appertured to provide a smoother and larger illumination spot. The grid spacing was determined more precisely to be $47.1 \mu \mathrm{m}$ in a separate measurement (discussed in section $\left.*^{*} ?^{* *}\right)$ using the transverse interferometry line. The lower magnification in this optical setup allowed imaging of the mesh and the mounting ring simultaneously, allowing an accurate calibration of the mesh spacing by comparison with the dimensions of the ring, (ID $2.29 \mathrm{~mm}$ ), which were measured precisely with a vernier 


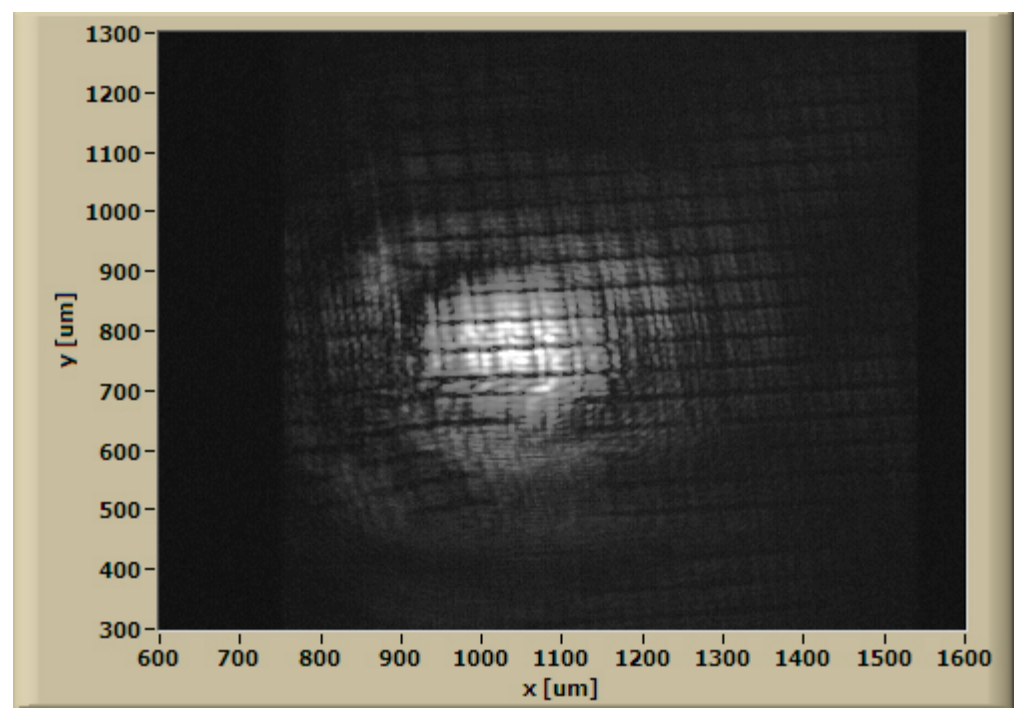

Figure 2.37: Image of 50 micron mesh using probe

calipers. The calibration of the probe image line was then done by choosing two points on the image vertically separated by 10 grids (it is the vertical imaging that is of primary concern here), and comparing the known size to the number of pixels spanned on the image. Since the image of the grid was tilted, it was necessary to assume that the horizontal and vertical calibrations are the same, which is usually a good assumption. The two points had a pixel separation of $\Delta x=26$ pix and $\Delta y=291$ pix, so that the diagonal distance was:

$$
\Delta s=\sqrt{(\Delta x)^{2}+(\Delta y)^{2}}=292.15 \text { pix }=10 \text { grids }=471 \mu \mathrm{m}
$$

yielding a spatial calibration of $1.61 \mu \mathrm{m} /$ pixel. This is close to the predicted value of $1.4 \mu \mathrm{m} /$ pixel. 


\subsubsection{Field of View}

Accounting for the spatial calibration of the imaging system, the 1040 pixels of vertical extent of the CoolSNAP CCD chip provide a vertical field-of-view of approximately $1.7 \mathrm{~mm}$. Since the ionization column and wakefield features that were measured all had transverse sizes less than $200 \mu \mathrm{m}$, the field-of-view was not a limiting factor.

\subsubsection{Choosing the Object Plane}

The goal of choosing the object plane of the imaging system is to find the location during the propagation of probe pulse at which the spatio-temporal phase features encoded in the field of the probe most closely represent the physics of the interaction. When imaging a 2-dimensional object, the location of the object plane is trivially determined as plane of the object itself. Any deviation from this location will cause the image morphology to differ from that of the object as a result of diffraction of the light being used to illuminate the object. The smaller the feature-size in the object, the more quickly diffraction will cause significant distortion. In the case of a laser interaction, there are several subtleties that complicate the choosing of the object plane. First is the fact that the object being measured is effectively threedimensional. During the transit of the probe through the gas jet, it experiences a wakefield structure that varies throughout the propagation. The variations include phenomena such as growth and decay of the wakefield, wave-breaking, beam loading and pump depletion which are a result of the complex interplay between the dynamics of the laser pump, the wake and the electron beam. The probe records the integrated effect of this varying structure in its phase. Because there is no one unique structure, there is no unique object plane. Instead there are a set of structures corresponding to a set of object planes, each intersecting the interaction volume at a unique z-position. What would be ideal would be to have the ability to 
image each of these distinct planes independently, and thereby resolve the evolution of the structure throughout the interaction. However, there are two things which make it difficult to do this. The first is that the phase accrued by the probe pulse accumulates through the interaction. The second is that the plasma and the density oscillations generated within it act like an optical element, distorting the image of the light traversing through it. This akin to trying to image a coke bottle by sending a beam through it. The bottle itself distorts the beam being used to image it, so an image of the back of the bottle (side nearest to the light source) is distorted by passage through the rest of the bottle, and an image of the front of the bottle has the intensity/phase modulations accumulated from the first part of the propagation. One way around this limitation is to use confocal microscopy in which a very low f-number imaging geometry is used to select the plane of interest, as is done in cell microscopy.

In the absence of the ability to resolve intermediate interaction planes, the object plane must be chosen to be immediately after the last plane which contributes to the signal (i.e. the output of the gas jet). The image that is taken then represents the accumulated effect of the whole interaction on the probe pulse, and thus only resolves features that are consistent throughout the interaction (e.g. linearly driven or mildly nonlinear wakefields). This is like imaging a bottle that is square and has a consistent wall thickness. The inevitable variations in the structure, for example at the entrance and exit regions of the interaction where the gas density is changing, will cause the measured structure to always deviate in some degree from the morphology of the wakefield inside the interaction. In addition, because the interaction length may be considerable, e.g. if the f-number of the pump beam is large, or if the interaction takes place in a channel, diffraction effects may also become significant. Just as in the case of a 2-D object as described above, displacement of the object from the object plane cause phase features to diffract and thereby spatially average. 
In the 3-D case, each of the interaction planes is separated by a different amount from the object plane at the output, and thus features accumulated from each plane suffer a different degree of diffraction. The severity of this effect can be estimated by defining an effective Rayleigh range for the features being measured. If the transverse structure changes on a spatial scale given by $w_{0}$, then diffraction of this feature will take place over a longitudinal distance given by $z_{\text {eff }} \cong \pi w_{0}^{2} / \lambda$. As long as the interaction length is comparable to or shorter than this effective Rayleigh

distance, $L_{i n t} \lesssim z_{e f f}$, then diffraction of the features imprinted on the phase of the probe will not be too severe. For an unchannelled experiment, the size of the wakefield features will generally be comparable to the size of the focus of the driving beam, and so the interaction length is naturally matched to the Rayleigh range of the features. If strong wake evolution (e.g. wave-breaking) were to occur, however, then density fluctuations would be created on a spatial scale determined by the physics of the process, and could potentially be much smaller than the spot-size of the laser. In this case these features will be more susceptible to spatial averaging by diffraction.

\subsection{Spectrometer Setup}

Because the spectrometer is the single most important detection system in FDH, its optimization can be critical to the success of the experiment. There are several important considerations when setting up the spectrometer detection system. Among these are: 1. choosing a CCD camera 2. Choosing a grating 3. adjusting the imaging of the slit 4 . choosing a slit width 5 . performing a spectral and spatial calibration of the system. The important specifications of the CCD camera are bit depth, number of pixels, linearity and dark current (i.e. noise). In general, it is best to choose a CCD with the greatest bit-depth available. Too few bits will lead to a high percentage of noise contributing to the signal from dark current, a 
low dynamic range (which may result in not being able to capture all the data in a given data run without changing the illumination), and discretization errors. In the experiments described here, a 12 bit camera was used, which is a minimum of desirable bit depth. The number of pixels sets the limits on the temporal resolution of the measured signal and the pulse separation of the probe and reference. This is because we will choose the dispersion of the grating so that the spectrum fills the CCD chip in the spectral axis, leaving just enough room on either side of chip for the intensity to descend below the noise level. This prevents loss of information and the introduction of noise in the spectral analysis from discontinuities in the amplitude. Once the spectral dispersion is determined, the maximum pulse separation between the probe and the reference pulses is limited by the spectral resolution of the spectrometer. This is because the fringe separation of the interferogram is inversely proportional to the pulse separation. The spectral period of the fringes must be several times larger than the spectral resolution of the spectrometer. In order to increase the spectral resolution of the system without diminishing the spectral bandwidth, the only solution is to increase the number of pixels in the wavelength axis.

It is important that the response of the CCD be linear as any deviation in linearity will result in a distortion of the shape of the fringes which will represent a significant source of phase noise. Once a CCD is chosen, a matching grating must be chosen as indicated above. First, the groove density must be chosen so that the full bandwith of the probe and reference is mapped onto the CCD, leaving space on either side for the signal to drop to zero. In addition, the groove density and blaze wavelength strongly determine the efficiency of diffraction of the probe light into a given order. To properly set up the detection system, it is thus necessary to characterize the efficiency of the grating and choose the blaze wavelength appropriately to optimize the amount of signal received on the CCD. 


\section{Chapter 3}

\section{Chirped Frequency Domain Holography}

The Frequency Domain Holography diagnostic consists of having two probe pulses propagating colinearly with the plasma structure to be measured. The first pulse acts as the recording medium, storing the density profile of the structure as a timevarying phase-shift of the optical field induced by a corresponding variation in the index of refraction of the plasma, which in turn is correlated to the electron density. For the phase shift to occur, this "probe" pulse must copropagate with the structure, overlapping with it both temporally and spatially. The recorded signal is the accumulated phase shift accrued over the whole interaction, and thus any relative motion between the probe and the structure during the interaction causes a blurring or temporal averaging of the signal. The second pulse acts as a phase reference with which to interfere the probe in the spectrometer, and thus must ride ahead of the plasma disturbance to ensure that it has pristine optical phase, unaffected by the interaction. 


\subsection{Designing the probe}

In principal, the only requirement on the probe pulse is that it be temporally long enough to record the region of interest. This can be achieved in one of two ways: the probe can be transform-limited and have a narrow spectrum, or it can be chirped, and have a broader spectrum. The chirped pulse presents an advantage, however. In general, to resolve a temporal feature with an optical pulse, there must be sufficient bandwidth to satisfy the time-bandwidth product for that feature. In the case of a transform-limited, narrow-band pulse, the spectrum is initially too narrow to resolve temporal features on a sub-pulse duration scale (by definition). However, the very interaction of the probe with the plasma structure introduces new frequency components into its spectrum by the process of temporal phase modulation. As a result, the interacted probe will always have sufficient bandwidth to support the temporal resolution of whatever features are imposed upon it. However, in practice, the interaction of the probe with a plasma may generate only weak spectral components, and since FDH is a phase-based technique, it is important that the spectral amplitude be large enough to allow the phase to be well defined and therefore relatively noise-free. If the probe pulse is chirped, on the other hand, it can have a very broad spectrum and still be temporally long. In this case, the phase modulations are recorded in a part of the spectrum which is strong, and the phase can be accurately reconstructed. The only requirement on the reference pulse, then, is that it have sufficient bandwidth to interfere with all relevant spectral components of the probe. In principle the reference need not be chirped, but in practice, it is best to have the chirp on the reference be similar to that of the probe, so that the spectral

phase of the interferogram not be dominated by the phase term corresponding to the relative-chirp. 


\subsection{The reconstruction process}

In Frequency Domain Holography, the information about the interaction is encoded in the temporal phase of the probe optical field. The basic strategy used to recover this information is thus to fully reconstruct the electric-field of the probe pulse, using the hologram and auxiliary measurements, and then extract the temporal phase. The word reconstruction is used for this process because different components of the electric field are measured separately using independent techniques, and the resulting information is then pieced together to generate a complete description of the field. These measurements are necessarily done in the spectral domain since there is obviously no equipment with sufficient temporal resolution to directly measure the temporal behavior of the field directly. Once the electric field is assembled in the spectral-domain, it is then simple to recover the temporal field, and thus the temporal phase, by Fourier-transforming to the time-domain.

Starting with the description of the field in the spectral-domain, the electric

field can be written: $E_{\text {probe }}(\omega)=\left|E_{\text {probe }}(\omega)\right| e^{-i \phi_{\text {probe }}(\omega)}$, where $\left|E_{\text {probe }}(\omega)\right|^{2}$ is the power-spectrum of the probe, and $\phi_{\text {probe }}(\omega)$ is the spectral phase. The spectral phase can be broken down into three main contributions: the chirp of the probe pulse, the interaction with the wakefield (i.e. the signal we are trying to measure), and the carrier phase: $\phi_{\text {probe }}(\omega)=\phi_{\text {probe chirp }}(\omega)+\phi_{\text {signal }}(\omega)+\omega t$. The last term only serves to identify the optical frequency of the field, and so it is not necessary to keep track of it during the calculation.

As a naïve first impression, one might expect that the temporal phase could be recovered directly from knowledge of the spectral phase. However, as is well known, phase and amplitude do not transform independently. It is useful to interpret this fact from a physical perspective. As already stated, the signal is encoded as a temporally-varying phase shift to the optical field of the probe. But a temporallyvarying phase shift is physically equivalent to a change in the rate at which the 
optical field cycles. In other words, these phase shifts modify the local carrier frequency or rather, the optical wavelength of the light. The process of interacting with the wakefield therefore causes a modification of the probe spectrum. For sufficiently strong interactions, the spectrum can be significantly altered, eliminating spectral amplitude at some wavelengths, and generating amplitude at new wavelengths. To reconstruct the probe field, we must therefore determine not only the spectral phase of the probe (from the two relevant sources described above), but also the post-interaction (modulated) power-spectrum of the probe.

Most of the information about the probe field can be determined from the Frequency Domain Hologram, which is simply a spectral interferogram generated by the interference of the probe and reference pulse fields in the spectrometer. The interferogram can be written:

$$
S_{F D H}(\omega)=\left|E_{p r}(\omega)\right|^{2}+\left|E_{r e f}(\omega)\right|^{2}+\left|E_{p r}(\omega)\right|\left|E_{r e f}(\omega)\right| e^{i \Delta \phi(\omega)}+c . c .
$$

where $\Delta \phi(\omega)=\phi_{\text {pr }}(\omega)-\phi_{\text {ref }}(\omega)=\Delta \phi_{\text {chirp }}(\omega)+\phi_{\text {signal }}(\omega)+\omega \Delta t$ is phase difference between the probe and the reference pulses, and $\Delta \phi_{\text {chirp }}(\omega)$ is the difference in the amount of chirp in the two pulses, and $\omega \Delta t$ is the carrier-phase contribution from two pulses separated in time by $\Delta t$. Clearly, extracting information about the probe from the interferogram would be trivial if each of the terms could be independently determined. Fortunately, we can nearly do that. Upon inspection, the interferogram is composed of two terms that are oscillatory (third term and complex conjugate), representing the interference of the two fields, and two that are not (first and second terms), representing the power spectra of the two fields. This distinction allows us to apply a Fourier Transform to the interferogram which separates the oscillatory terms from the non-oscillatory ones. The Fourier Transform generates a time-domain signal with four peaks corresponding to the four terms of the interferogram. The first two terms both transform to peaks at $\tau=0$, and 
the oscillatory term and its complex conjugate transform to peaks at $\tau=\Delta t$ and $\tau=-\Delta t$ respectively. ( ${ }^{* *}$ graph ${ }^{* *}$ ) Assuming that the period of the interference pattern is sufficiently small compared to the amplitude variations of the spectrum, the side peaks will be fully separated from the D.C. central peaks. This lack of overlap allows us to apply a filter to the FFT signal that passes one of the two side-peaks without contribution from any other term. Inverse transformation of this filtered signal then yields the contribution to the hologram from the oscillatory term by itself, i.e. $S_{\text {term } 3}(\omega)=\left|E_{p r}(\omega)\right|\left|E_{r e f}(\omega)\right| e^{i \Delta \phi(\omega)}$. From this term we can with some additional information extract the desired quantities $\left|E_{p r}(\omega)\right|$ and $\phi_{\text {signal }}(\omega)$.

In order to retrieve the modulated spectral amplitude of the probe, $\left|E_{p r}(\omega)\right|$, we must first independently measure the power-spectrum of the reference pulse: $\left|E_{r e f}(\omega)\right|$. Then the modulated power-spectrum of the probe is retrieved as: $\left|E_{p r}(\omega)\right|=$ $\left|S_{\text {term } 3}(\omega)\right| /\left|E_{\text {ref }}(\omega)\right|$. In order to extract the signal phase, from $S_{\text {term } 3}(\omega)$, we also need to independently measure $\Delta \phi_{\text {chirp }}(\omega)$, the difference in chirp between the probe and reference pulses. This can be done simply by measuring a reference interferogram with no gas or pump pulse present. The resulting phase difference will then be limited to: $\Delta \phi_{\text {reference }}(\omega)=\Delta \phi_{\text {chirp }}(\omega)+\omega \Delta t$. The signal phase is then recovered as: $\phi_{\text {signal }}(\omega)=\Delta \phi(\omega)-\Delta \phi_{\text {reference }}(\omega)$.

\section{3 measuring probe chirp}

The overall chirp of the probe pulse can be determined in more than one way. I will outline two methods that were used which gave comparable results. The first was described by Howard Milchberg's group in their paper entitled "Single-shot Supercontinuum Frequency Domain Holography." [ref ?] In this method, a thin coverslip of glass is placed near enough to the focus that the pump will induce a nonlinear index of refraction in it (** equation for nonlinear index of refraction here **). The probe, overlapping the pump spatially and temporally will experience this 
change in the index of the glass as a temporal spike in its phase. This process is known as cross-phase modulation (XPM). The phase shift experienced by the probe pulse will then reflect the temporal profile of the pump. As a result, because the pump pulse is short $(\sim 30 \mathrm{fs})$, the rate of change of the induced phase shift, will be comparable to the optical frequency of the pulse $\left(d \phi / d t \sim \omega_{0}\right)$, which means that the spectrum of the probe will be significantly modified. For the case of a chirped probe modulated by a gaussian pump pulse, a symmetric pattern of ripples will be imposed on the probe spectrum. (** graph here ${ }^{* *}$ ) The spectral point of symmetry is set by the relative delay of the pump and probe. Since the probe is chirped, there is a (nearly linear) correspondence between the instantaneous wavelength of probe field, and this delay. The symmetry point is then set by the wavelength of the probe which overlaps the pump during the interaction. By scanning the pump-probe delay, we can measure the dependence of the wavelength of the symmetry point as a function of delay and thus map out the time-wavelength correspondence that is the chirp of the probe pulse. (** graphs of modulation vs. delay and corresponding reconstructed time-spectrum curve $\left.{ }^{* *}\right)$ The spectral chirp, $\phi_{\text {probe chirp }}(\omega)$ can then be calculated directly from this curve.

The second method to calculate the probe chirp is considerably more straight forward. In this method, a thin doubling crystal is temporarily inserted into the pump line to generate a transform-limited reference pulse at the second harmonic. This pulse is then interfered with the chirped probe in the spectrometer. The interferogram then directly encodes the information about the difference in spectral phase between the two pulses. Provided that the reference pulse contains negligible spectral phase of its own, the recovered phase difference will be directly the spectral chirp of the probe plus a linear component from the temporal separation, which can be easily subtracted off. The advantage of this second technique is that it is singleshot, so that it can be done very quickly, and can be redone easily if the probe chirp 
is varied. The analysis is also considerably easier and can be done in real-time. The only potential drawback is if the reference pulse (in this case the pump), is not fully compressed, and has some residual spectral phase. Generally, this is not much of an issue as the residual phase in a compressed pump pulse is usually small compared to the chirp phase. Specifically in the experiments described here, the result of this method was close enough to that of the above one that there was no noticeable difference.

Once the probe temporal phase is known, the electron-density profile can be easily calculated. The accumulated phase is described by: $\Delta \Phi(\zeta, r)=\int 2 \pi \eta(\zeta, r, z) / \lambda d z$ the electron density is calculated directly from this by knowledge of the interaction length. Because the phase-shift is accumulated over the whole interaction, the recovered information represents an average of the structure in the comoving frame throughout the interaction. 


\section{Chapter 4}

\section{Wakefield Measurements}

\subsection{Initial Attempt with Photon Acceleration}

In the first round of experiments with the HERCULES system, an attempt was made to detect wakefields using photon acceleration. A single probe pulse was made to copropagate with the pump pulse, and be temporally overlapped with the wakefield. Although both relative pump-probe delay and plasma density were both scanned, no clear signature of a wake was observed. There was a strong spectral distortion at a delay corresponding to overlap of the probe with the ionization front (fig. 4.1). This distortion shows a radially varying degree of blue-shifting, some spectral modulation, and potentially a small amount of red-shifting as well. Though the modulation and red-shifting may be a result of interaction with a wakefield, the pattern of distortion is difficult to interpret.

The expected signature of the wakefield is a delay-dependent pure red- or blue-shift of the entire spectrum. This is what is expected if the pulse is short enough to reside within a single cycle of the wakefield (and able to stay in phase with it during the interaction). It is now understood that the reason for the lack of ability to resolve pure red and blue shifts is that the probe pulse was too long. As is described 


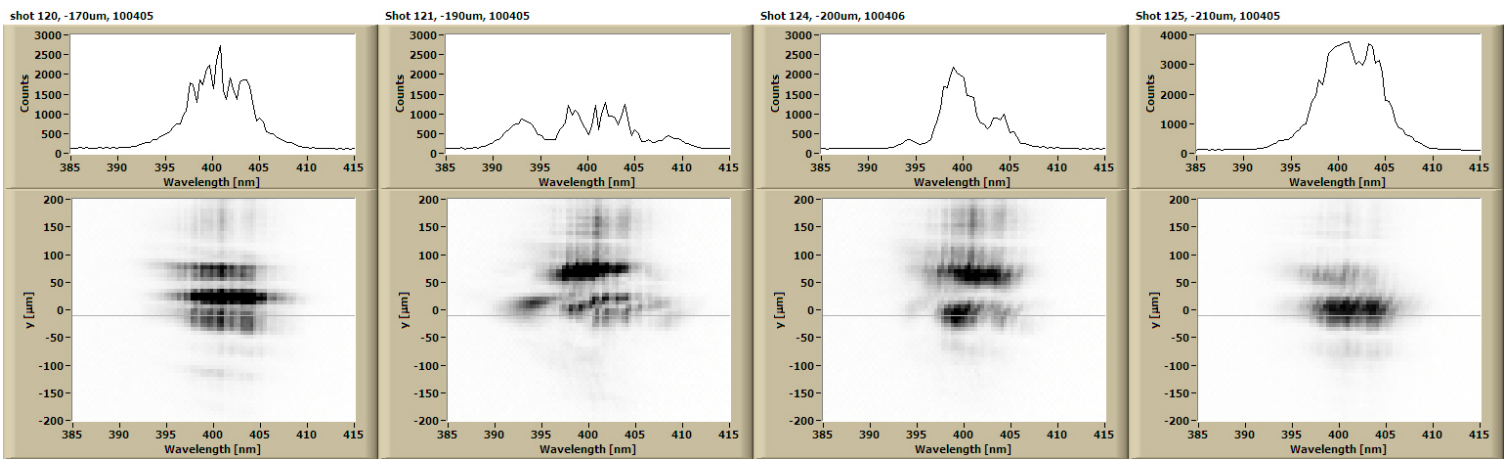

Figure 4.1: Red and blue-shifting of probe by ionization front and wakefield

in the probe-generation section, the second-harmonic probes were coupled with the pump through a 1" thick piece of glass. In the absence of compensation, groupvelocity dispersion in this optic chirps out the probes to nearly a picosecond. An attempt was made to pre-compensate this chirp by adjusting the compressor of the probe line. The probe compressor grating-separation was scanned while observing the duration of the coherence in an interferometric cross-correlator (fig. 4.2). A minimum was found at a grating separation of $\Delta G \approx 3 \mathrm{~mm}$ from the setting used to optimize the pulse duration of the probe before going through the beam-splitter. This figure of $3 \mathrm{~mm}$ agrees roughly with analytical calculations that were done to simulate the precompensation procedure.

The compensated pulse duration of the probe was measured at $\Delta G=3 \mathrm{~mm}$ using the interferometric cross-correlation technique described in section 3.2 .2 (fig 4.3). Although the interferometric measurement indicates a short, correctly precompensated pulse (of duration $\approx 45 \mathrm{fs}$, this result turned out to be misleading, and emphasizes the distinction between the coherence between the pump and probe, and the true intensity cross-correlation. A separate measurement was done with a thirdorder correlator, and the result is shown in figure 4.4. This measurement shows that the actual probe pulse duration was closer to $240 \mathrm{fs}$, and it was thus impossible to 


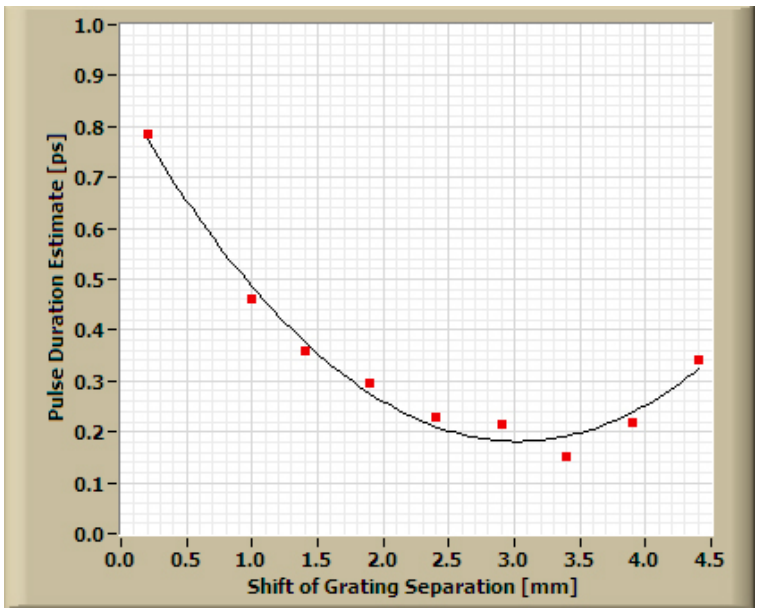

Figure 4.2: The probe compressor grating was scanned while observing the duration of the coherence spike of the interferometric cross-correlator.

resolve the spectral shifts from the wakefields at the densities examined.

A careful optimization procedure using the $3^{\text {rd }}$ order cross-correlator was then done to determine the shortest achievable probe pulse duration, and the result, shown in figure 4.5 was a pulse duration not shorter than 148 fs. Although this is better than the previous result, it is still too long to do the desired measurements. A plasma density between $2 \times 10^{17} \mathrm{~cm}^{-3}$ and $5 \times 10^{17} \mathrm{~cm}^{-3}$ would be required to be able to resolve the wakefields with photon acceleration using these pulses, and at these densities, the shifts would be very small.

It was clear from these pulse-duration measurements that another technique would be required to be able to resolve the wakefields. The technique that was chosen was chirped Frequency Domain Holography, in part because of the great increase in sensitivity to the structure of the wake, and also because the use of chirped pulses naturally and easily avoided the obstacle that was fatal to the photon acceleration experiment. 


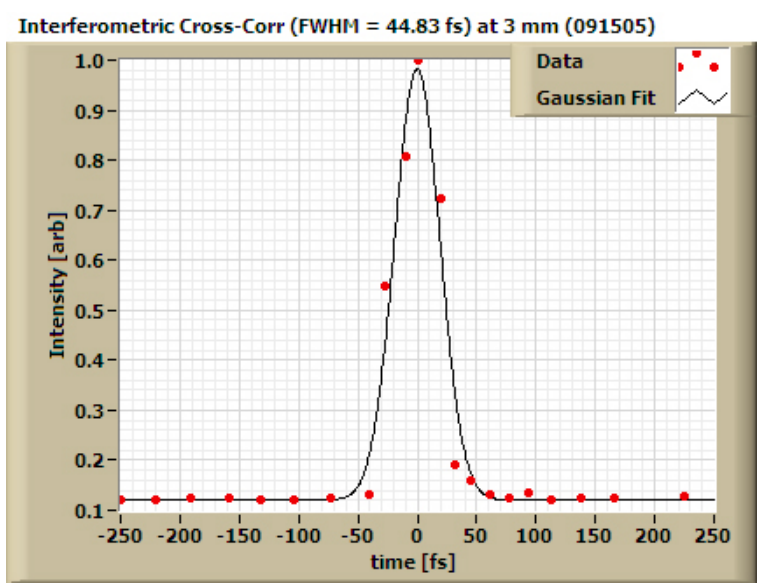

Figure 4.3: Interferometric cross-correlation between the pump and probe in the second harmonic. The Gaussian fit has a FWHM of $45 \mathrm{fs}$.

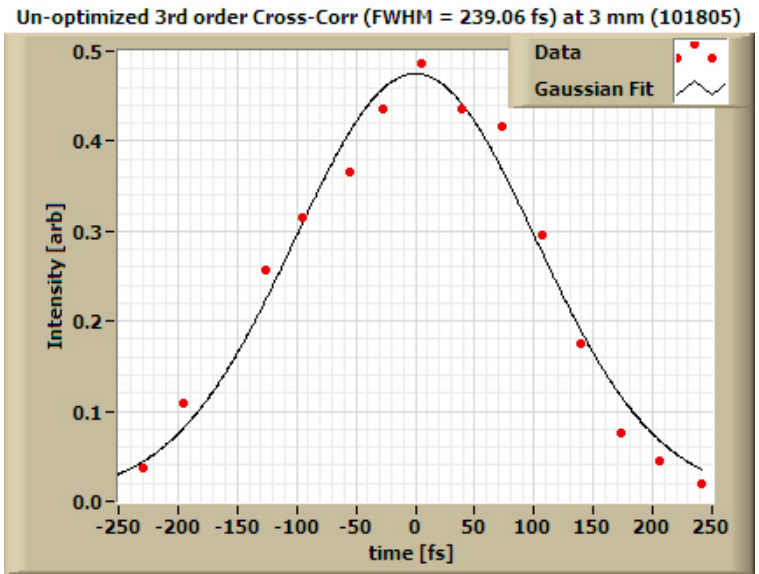

Figure 4.4: Interferometric cross-correlation between the pump and probe in the second harmonic. The Gaussian fit has a FWHM of $45 \mathrm{fs}$. 


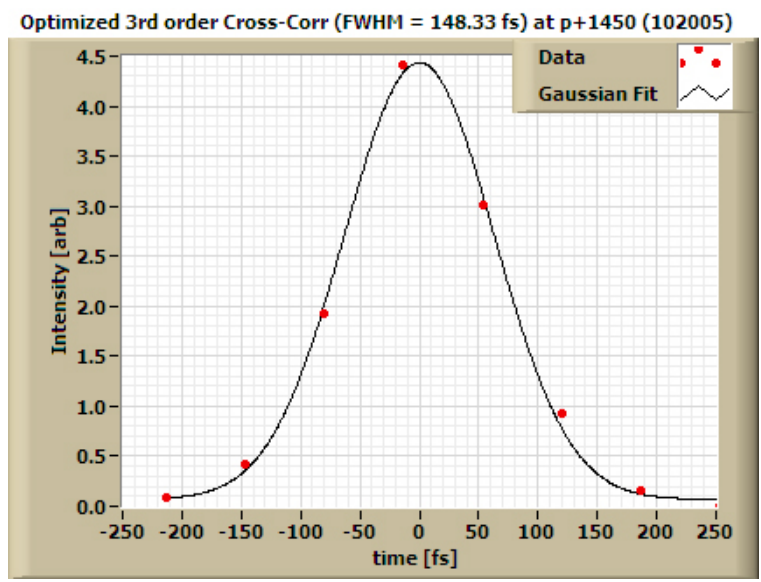

Figure 4.5: Optimized $3^{r d}$ order cross-correlation measurement of probe pulse. Lowest pulse duration achieved: $\Delta t_{F W H M}=148 \mathrm{fs}$ 


\subsection{First Wakefield Measurements, 11/21/05}

The first wakefield measurements were done on November 21, 2005. The online real-time LabVIEW analysis routine was still being debugged using $10 \mathrm{TW}$ shots when the oscillations were first noticed. Figure 4.6 shows one of the first wakefields ever observed.

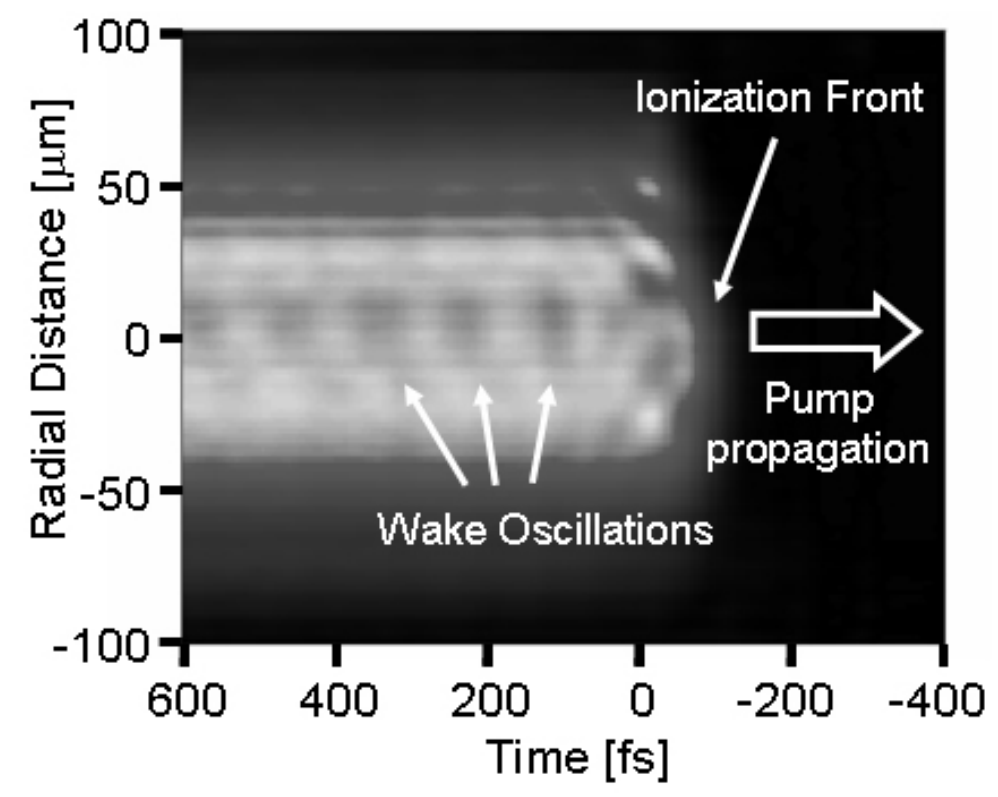

Figure 4.6: Density reconstruction of wakefield measured on Shot 048, 11/21/05.

Even the very first acquisitions taken with this system showed the presence of oscillations, but at a very low level. In order to determine if these oscillations were actually the result of wakefields and not due to a noise artifact, the density was then systematically scanned over the range where resonant wakefields were expected. The results are shown in fig 4.7.

As can be seen, the measured periodicity matches the expected exceptionally well over an electron-density range greater than an order of magnitude, confirming that the oscillations were actually measurements of wakefields. No fitting factors 


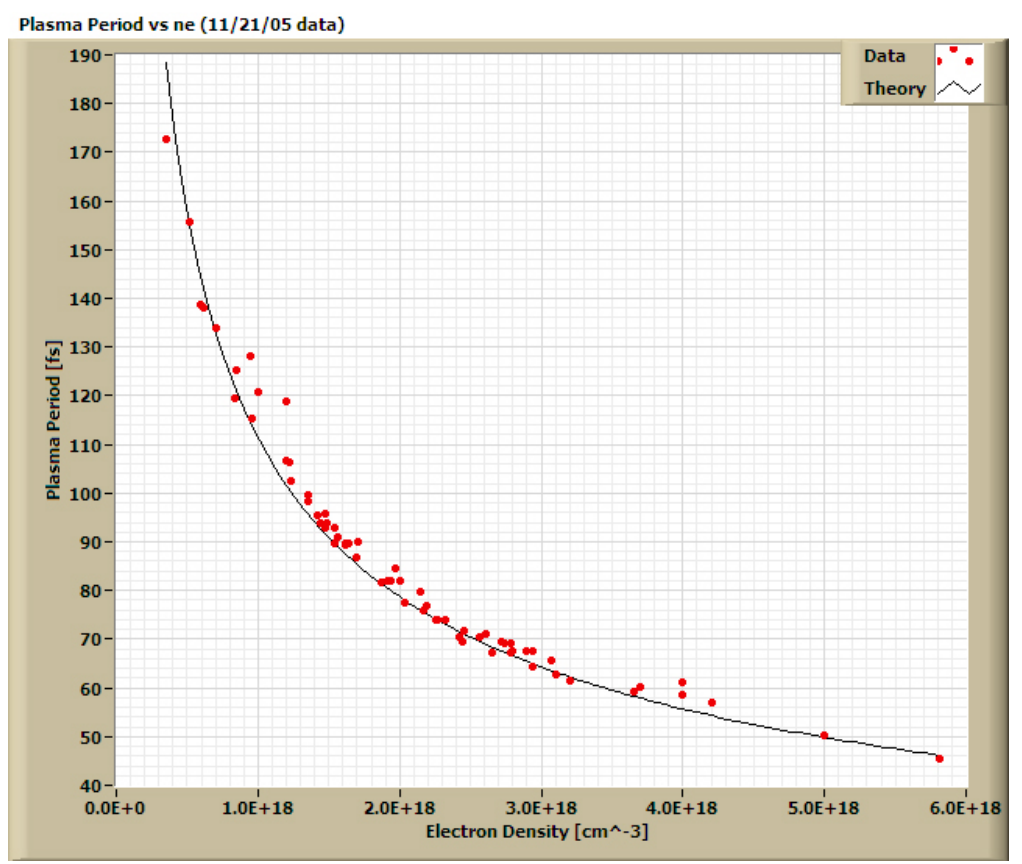

Figure 4.7: The dependence of the measured plasma period on electron density matches the theory extremely well over more than an order of magnitude of electron densities. These data were taken over a period of 2 hours.

were used. The periodicity was determined by manually measuring (using a cursor on the graph) the time difference between the crests of as many cycles of the oscillation as were well-defined. In some cases this number was as low as 3 cycles, and in other cases, as high as 8 . The total time was then divided by the number of cycles to give the wave period. The electron density was determined by measuring the phase-shift of the doubly-ionized plateau region (i.e. the total phase-shift due to the ionization front). This phase shift was then converted to an electron density by assuming a longitudinally-uniform plasma density profile with a length of $1.5 \mathrm{~mm}$ (this length having been determined by observation of one of the plasma profiles from the transverse interferometry). This assumption has some difficulties, as will be discussed in section (**?**), but works fairly well, nonetheless. Sample images 
of the wakefields measured in this run can be seen in table 4.1 and table 4.2. 
Table 4.1: Phase Images of Wakefields taken during pressure scan on 11/21/05
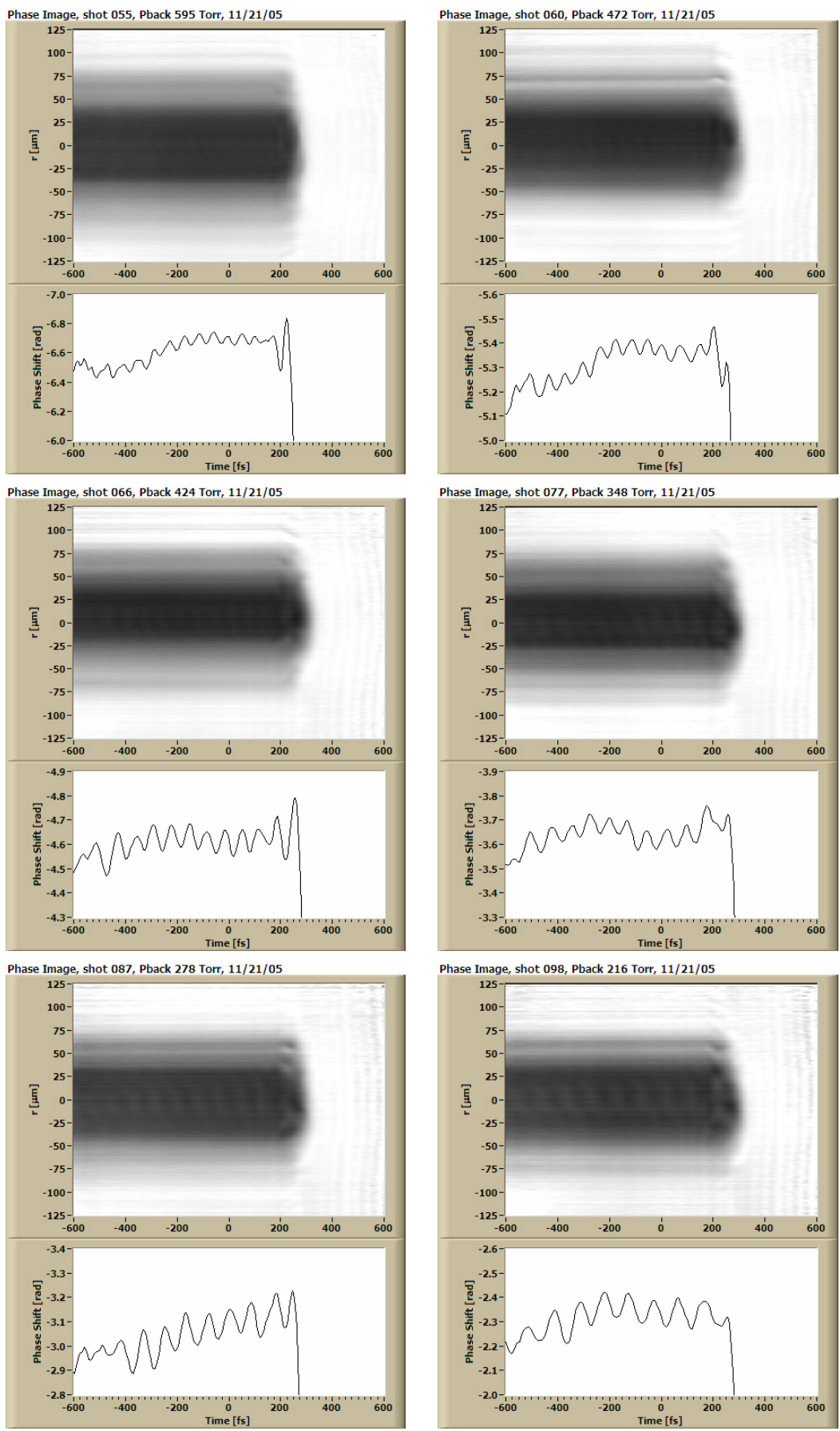
Table 4.2: Phase Images of Wakefields taken during pressure scan on 11/21/05
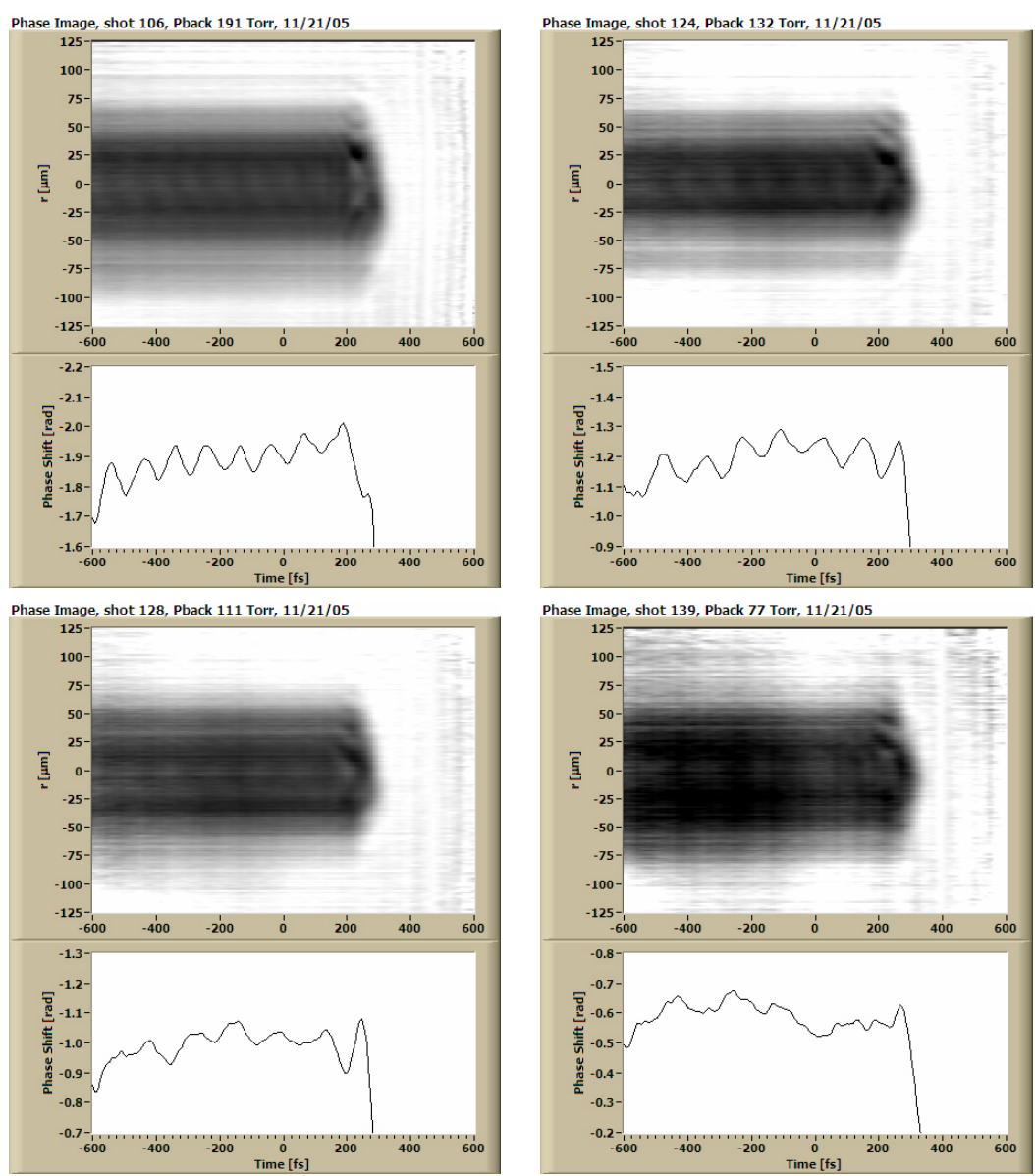


\subsection{First 30 TW shots, 11/22/05}

The following day, the laser power was increased from the $10 \mathrm{TW}$ level to the 30 TW level to examine the effect on the measured wakefields. The first shot, shown in figure 4.8 was excellent, showing stronger, higher amplitude wakes as expected in the higher power case. In addition, the wavefronts in this shot also demonstrate a strong degree of curvature, which is a consequence of being nonlinearly driven to amplitudes where relativistic modification to the plasma frequency begins to take place. This effect will be discussed in more detail in the analysis section.

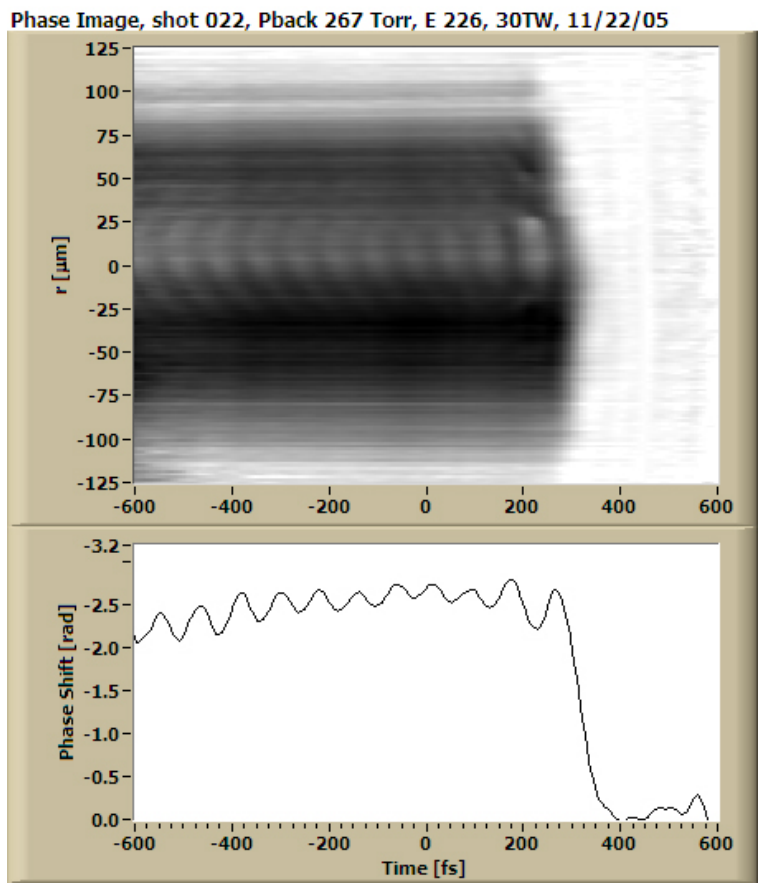

Figure 4.8: First 30 TW shot taken on 11/22/05.

Unfortunately, subsequent shots on this day were not quite as nice as the first. In spite of attempts to optimize the wake amplitude by scanning the image of the wake across the spectrometer slit, scanning pressure and jet location, and various other parameters, the amplitude of the subsequent wakes was not as high 
as that of the first, although the presence of strong curvature was still noticable. It was later determined that the $400 \mathrm{~nm}$ pick-off mirror used after the interaction to reflect off the probe pulses and pass the pump had become damaged. It is likely that this mirror damaged on the first 30TW shot, and that the shots following this one suffered in quality in some way that is not well determined. The following tables (tbls 4.3 and 4.4) shows a representative sample of the better images taken on this day. In the data corresponding to higher densities, several of the images show discontinuous features which are artifacts of the phase-retrieval procedure. When a phase image is constructed, it is always necessary to perform a procedure known as "unwrapping" which removes discontinuities that exceed pi radians. These discontinuities are generally unphysical, and are a consequence of the lack of determination of the argument of a complex number to within a multiple of pi. For higher densities, the phase-unwrapping algorithm has difficulty determining where to remove the discontinuities, since the phase gradients become large, and the result is bands of the image which are at a different level than the rest.

Table 4.3: Phase Images of 30TW Wakefields taken on 11/22/05
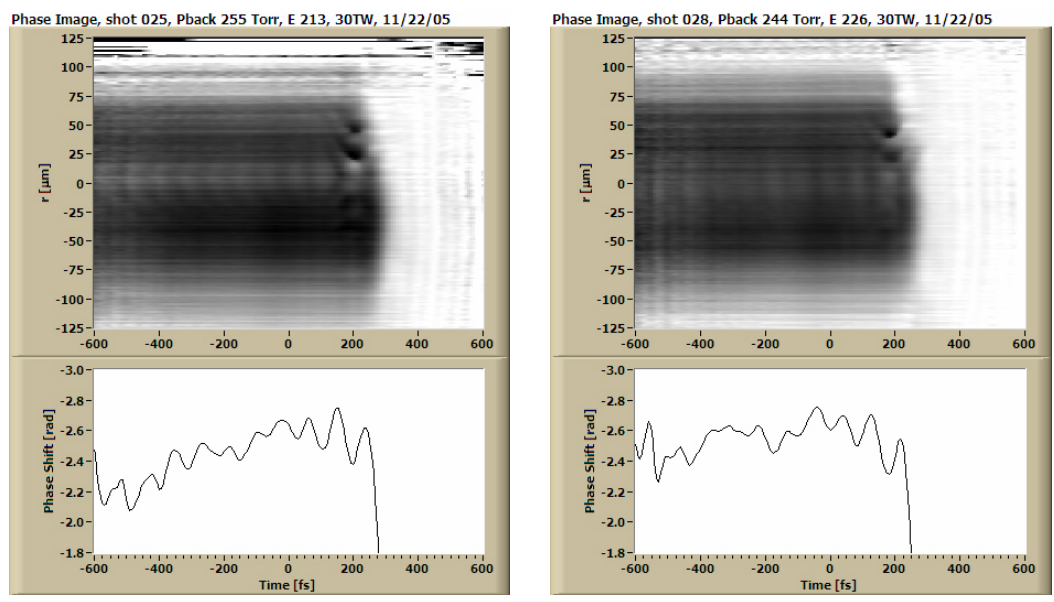
Table 4.4: Phase Images of 30TW Wakefields taken on 11/22/05
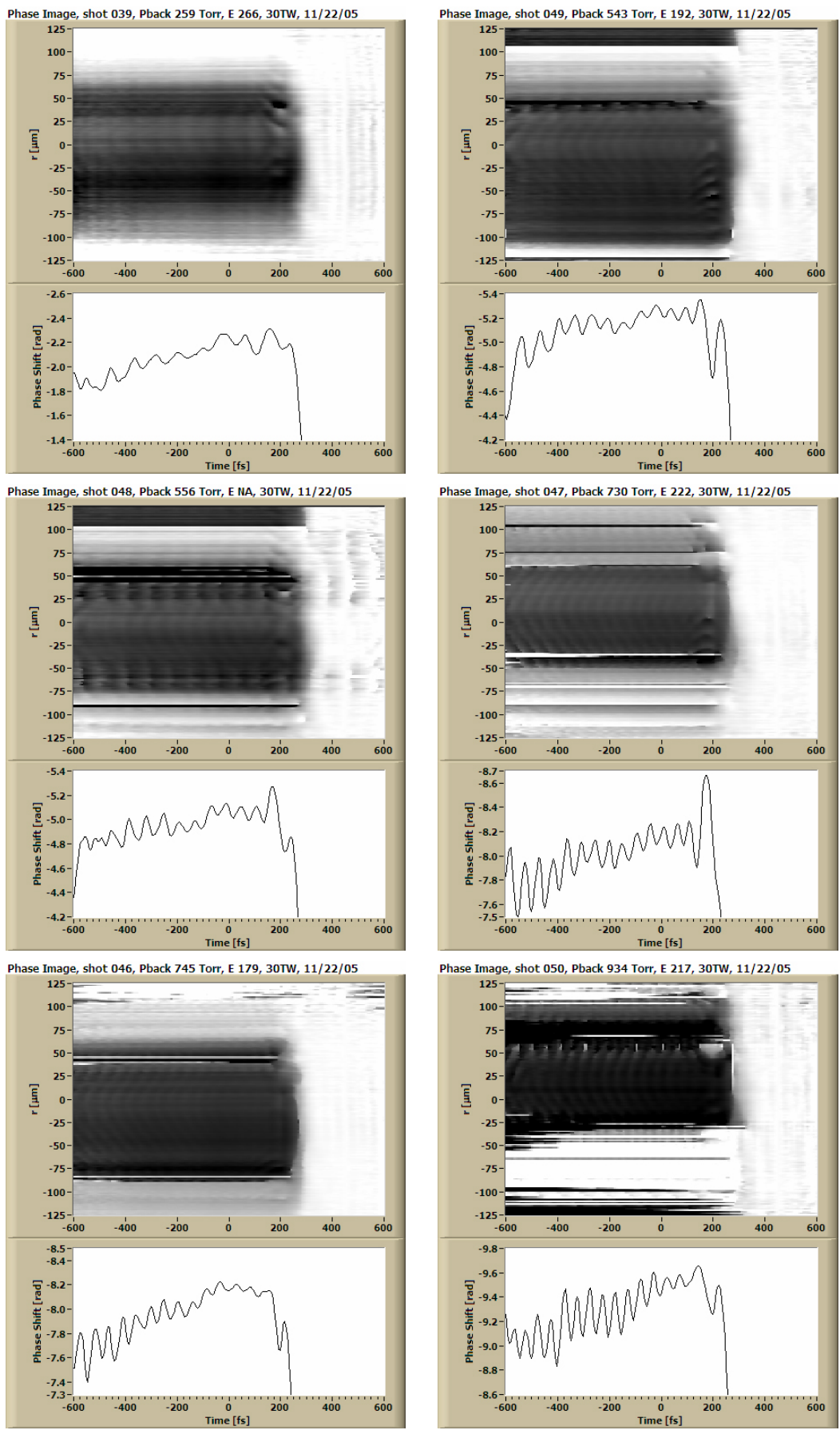


\subsection{2/13/05 Wakefields}

In the following run, shots were taken first at $10 \mathrm{TW}$, then at $12 \mathrm{TW}$, and finally at 30 TW, demonstrating a clear change in the amplitude and structure of the wakes produced. Although the need was clear for more reliable results at the $30 \mathrm{TW}$ level after the first run, difficulties with the laser system prevented taking more $30 \mathrm{TW}$ shots until December 13. In order to achieve $30 \mathrm{TW}$, the final amplifier is engaged, and the pumping of the crystal in the power-amplifier stage places a higher thermal load on the cryogenic cooling system ("Cryo-Tiger") for the crystals. Problems with the functioning of the Cryo-Tiger system often limited the time available for shooting, so it is for this reason that initial "10 TW" shots were done with the laser de-tuned from peak operation to finalize alignment and test for the appearance of wakefields. Table 4.5 shows a couple of these shots.

Table 4.5: Phase Images of 10TW Wakefields taken on 12/13/05. The laser was de-tuned, to prevent the temperature of the "Cryo-Tiger" from rising too soon.
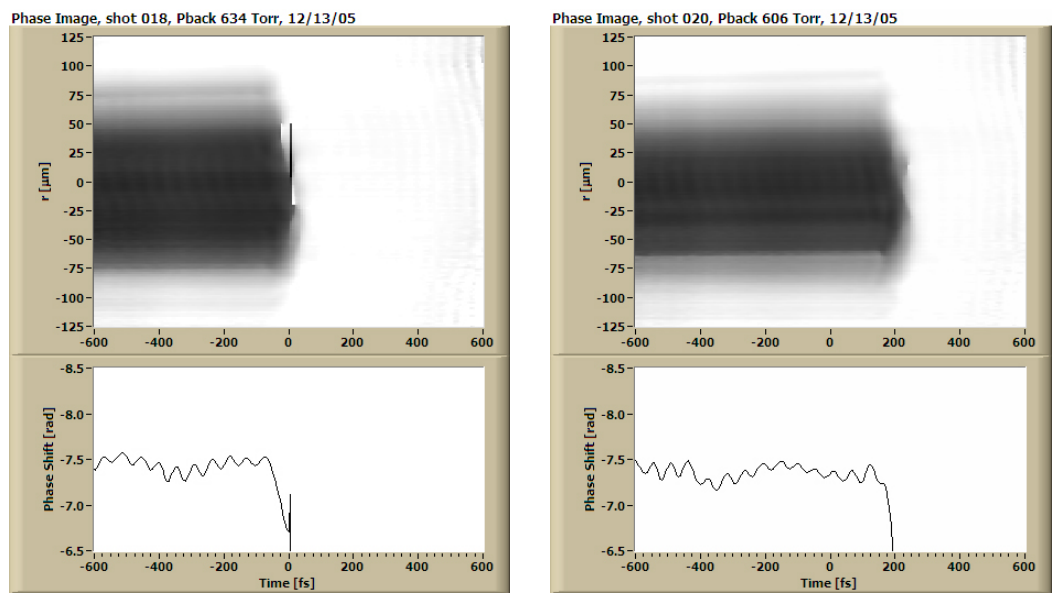

Once alignment and recovery of wakefields were established, the laser was then optimized at the $10 \mathrm{TW}$ level (no power-amp), to nearly $12 \mathrm{TW}$. The resulting wakefields show higher amplitudes and more clearly defined structures (table 4.6). 
Table 4.6: The laser optimized to nearly 12 TW. Wakefields show improved amplitude and clarity.
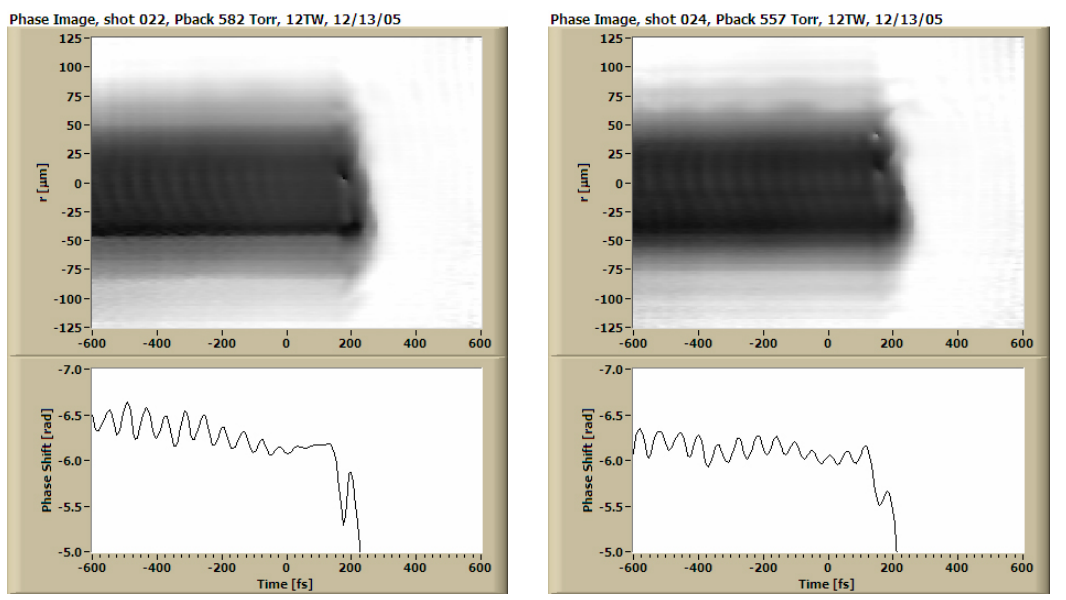

Finally, the laser operating at $30 \mathrm{TW}$ produces wakefields with noticably larger amplitudes and increased curvatures. A representative sample of the $30 \mathrm{TW}$ shots is shown in tables 4.7 through 4.10 . These shots show scans of both electron density and relative delay between pump and probes. The latter is important, because there may be some evidence that the amplitude of the recovered wakes depends artificially on the position of within the temporal window of the measurement, and not uniquely on the distance away from the pump pulse, for example, or the density of the gas or the power of the pump pulse. Further analysis is still necessary to resolve this issue. In the image titles, there is also an indication of the pulse energy as measured by a CCD camera. The energy is indicated by a number following the capital "E", and is on a relative, but arbitrary scale. 
Table 4.7: Wakefield images with the laser at $30 \mathrm{TW}$
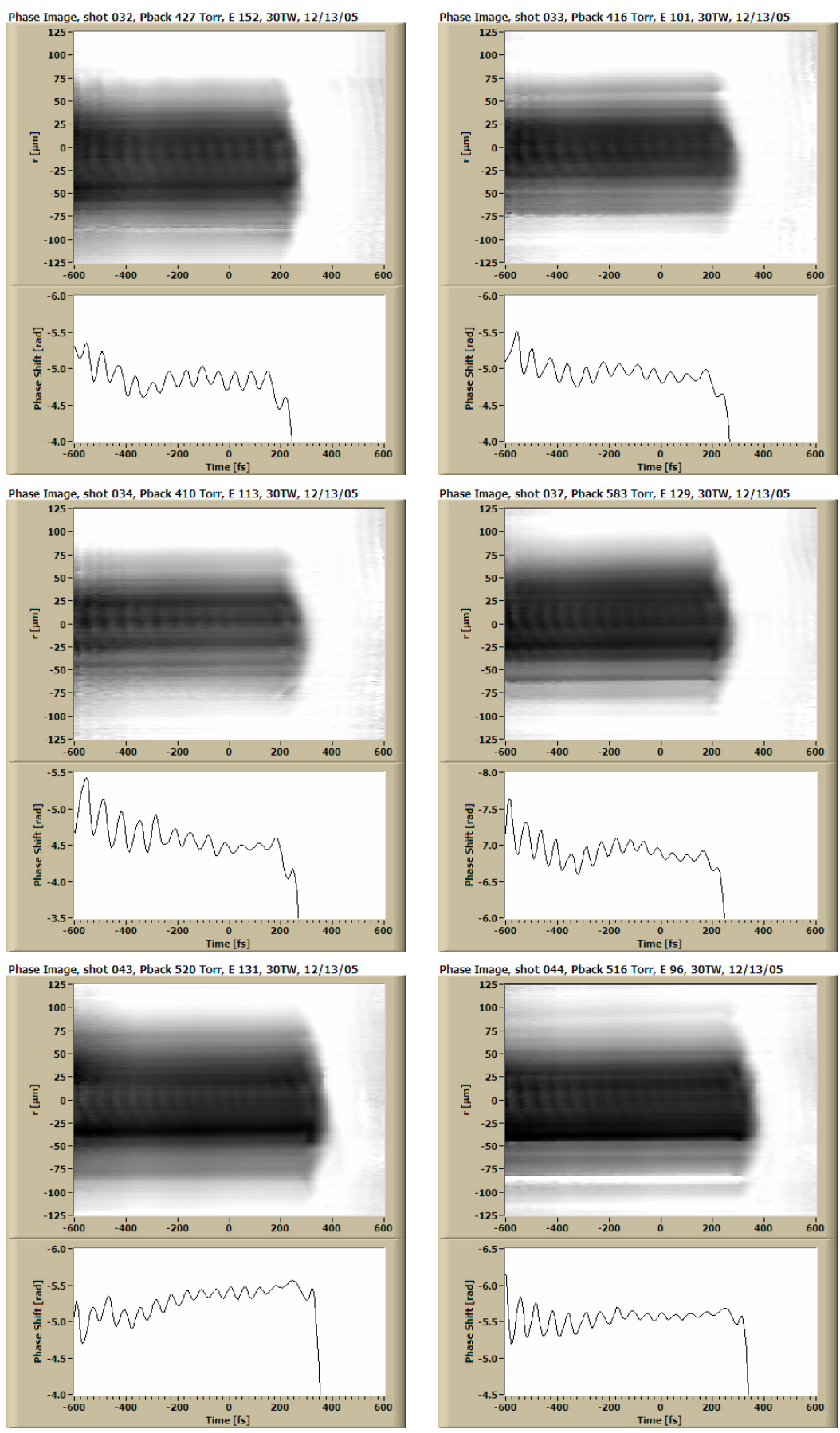
Table 4.8: Wakefield images with the laser at $30 \mathrm{TW}$
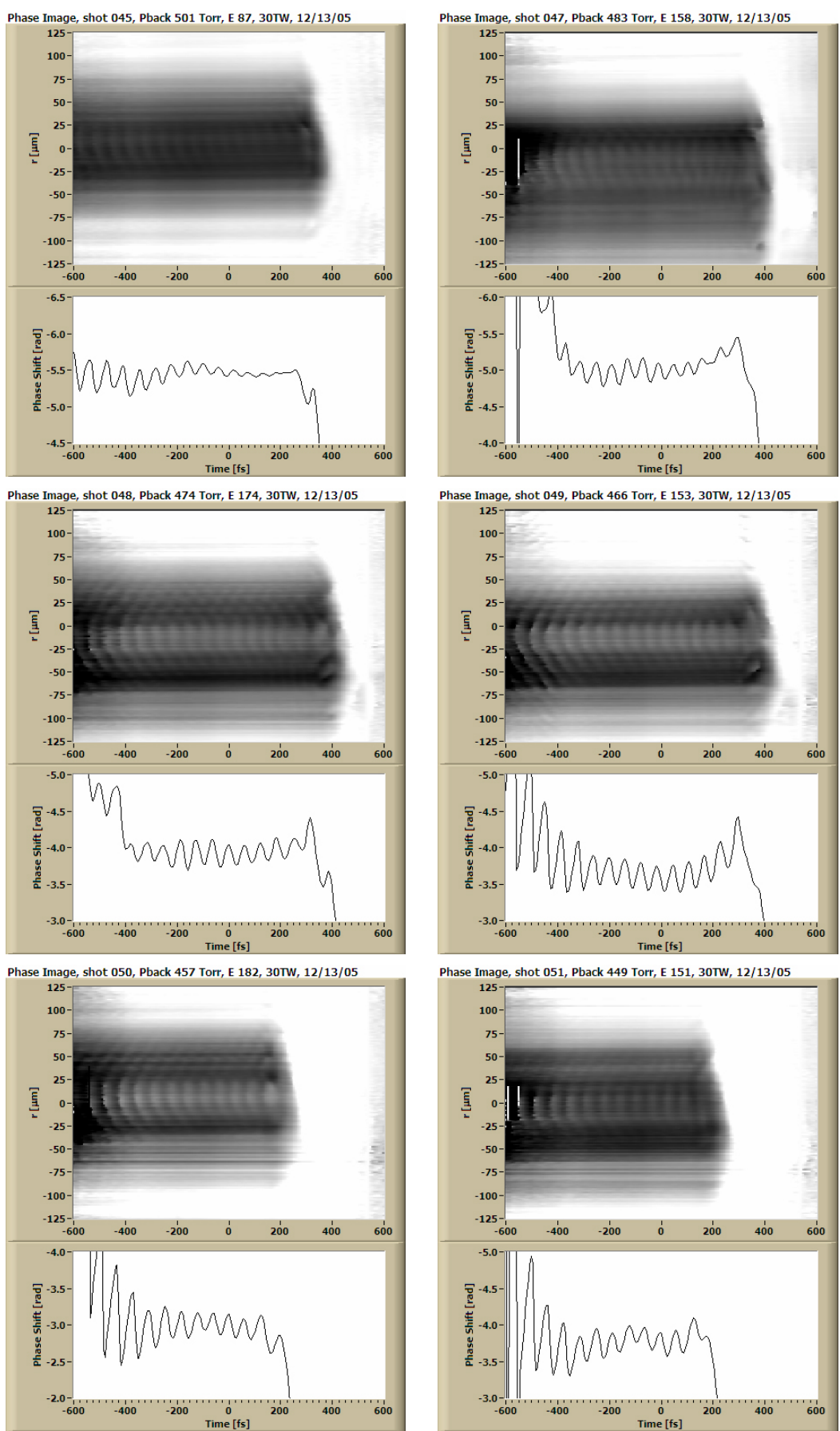
Table 4.9: Wakefield images with the laser at $30 \mathrm{TW}$
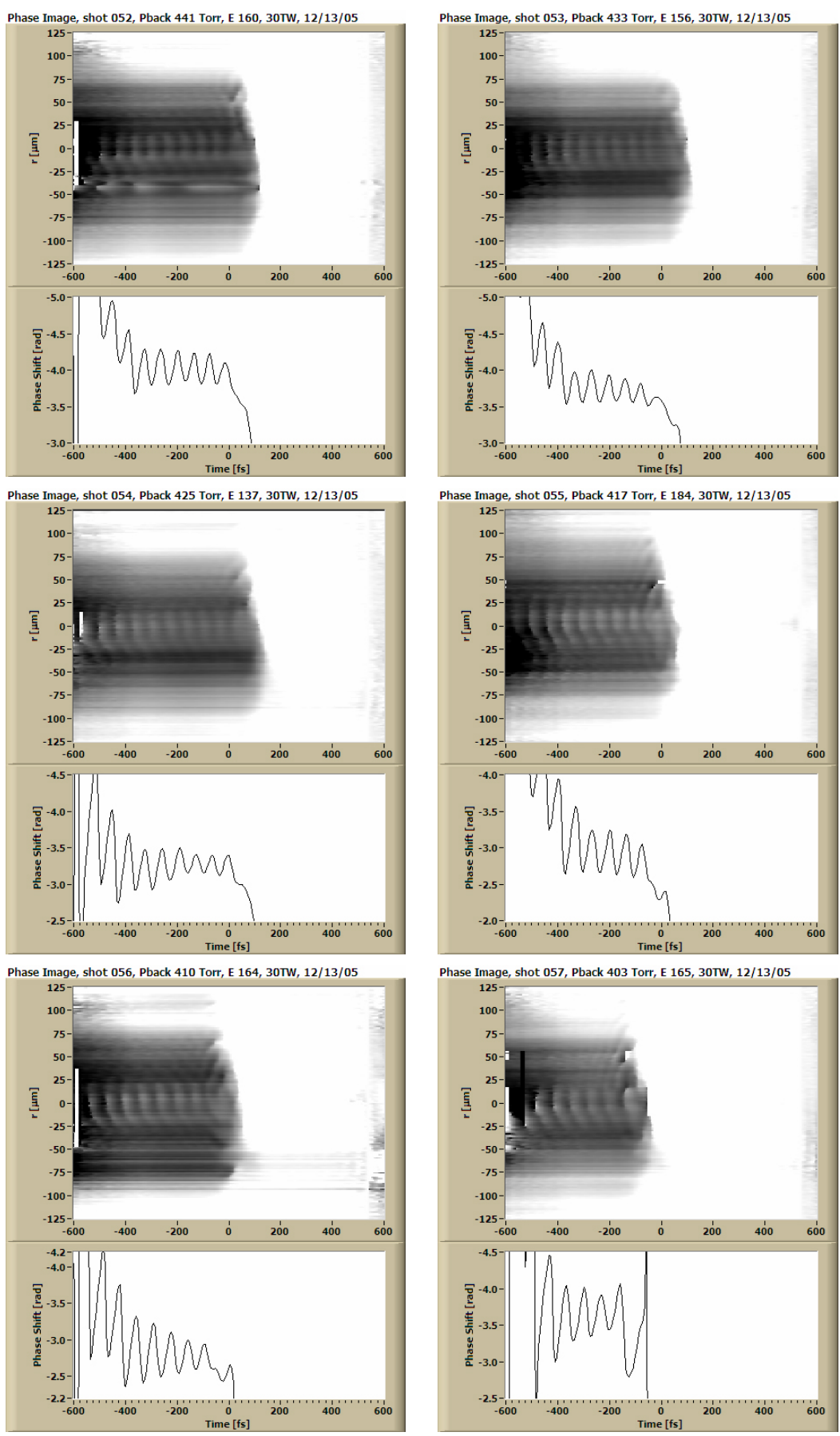
Table 4.10: Wakefield images with the laser at 30 TW
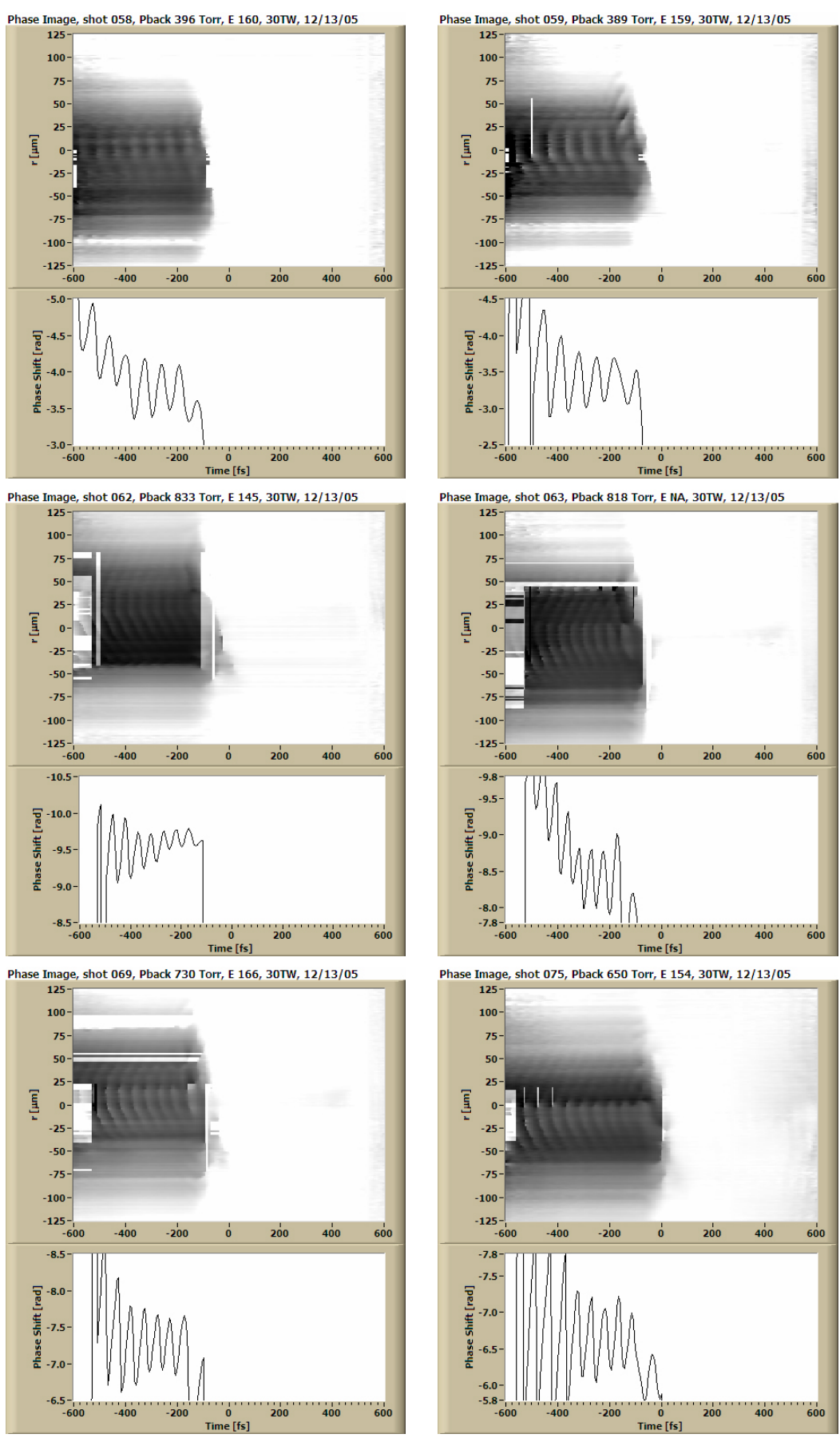


\subsection{Simulation and Analysis}

The wakefield images demonstrated above are striking in their level of detail, showing features such as wavefront curvature, variation in wake amplitude with distance from the pump pulse, radial amplitude variations and wavefront tilt, among others. Frequency Domain Holography thus promises to be an invaluable tool for studying the properties and behavior of wakefields. However, because this diagnostic is new, (at least in its application towards measuring wakefields), it is important to analyze and benchmark the technique against a known set of results. The first, most basic test is the check of the dependence of wakefield periodicity on electron density, which the measurements confirmed beautifully (fig. 4.7). But to characterize the fidelity of the reconstruction of the wake structure in the details such as wake amplitude and curvature, etc., is more difficult, because there are no other single-shot measurements of the wake structure. The only source of comparison is simulation. Although simulations have been successful in guiding researchers to the right experimental parameters for producing high-quality electron beams, there is still much that remains unknown about the details and sensitivity of the mutual interaction of the laser pulse, the plasma, and the electron beam under these highly dynamic conditions. For this reason, it is hoped that FDH will help provide a method for benchmarking simulations at the same time that simulation provides a sanity check for the measurements. Among the questions to be answered, are the following:

- Q1: What information does the diagnostic provide, and what information does it not provide?

- Q2: In what details, and to what degree can the measurement be expected to yield accurate results?

- Q3: What are the conditions under which the results are most accurate?

- Q4: How closely do simulations predict actual wake structures? 
In addition to providing a benchmark for simulation, it is also expected that FDH may provide a means for studying wakefield dynamics under conditions where simulations are not expected to be reliable, or when it simply takes too long for accurate simulations to run. In this section, a basic level of analysis and comparison with simulation is done for two of the measurements, a $10 \mathrm{TW}$ shot, and a 30 TW shot. Simulations were done with the code "WAKE." The code WAKE is a fully relativistic particle-in-cell (PIC) code that can be used in two-dimensional planar or three-dimensional axisymmetric geometry. It calculates the quasistatic plasma response to the ponderomotive force averaged over the laser period and to the self-consistent electric and magnetic fields of the plasma wake. Propagation of the linearly polarized laser beams is described in the extended paraxial approximation. Optical field (tunneling) ionization of neutral gas is included. The simulations presented here used grid size $\Delta \mathrm{x}=\Delta \mathrm{r}=0.16 k_{p}^{-1}, 15$ particles per cell, and a moving window $4.5 \mathrm{ps}$ long and $\sim 100 \mu \mathrm{m}$ wide. The technique originally designed for modeling multi-shot FDI [6] was adapted for FDH simulations. The code calculates precisely the linear group velocity of both $800 \mathrm{~nm}$ pump and $400 \mathrm{~nm}$ probe-reference pulses.

\subsubsection{Wakefield Morphology}

Initial comparisons were done by simulating the passage of the pump pulse through a trapezoidal electron density profile with density ramps $0.25 \mathrm{~mm}$ long, and a plateau region of $1.5 \mathrm{~mm}$. This profile was chosen as an estimate based on initial observation of the longitudinal density profile using the transverse interferometry diagnostic (fig.

Using the trapezoidal profile described above, simulations were done to compare with shot 048 at $10 \mathrm{TW}$, from 11/21/05, and shot 022 at $30 \mathrm{TW}$, from 11/22/05. These comparisons are shown in figures 4.10 and 4.11 . 


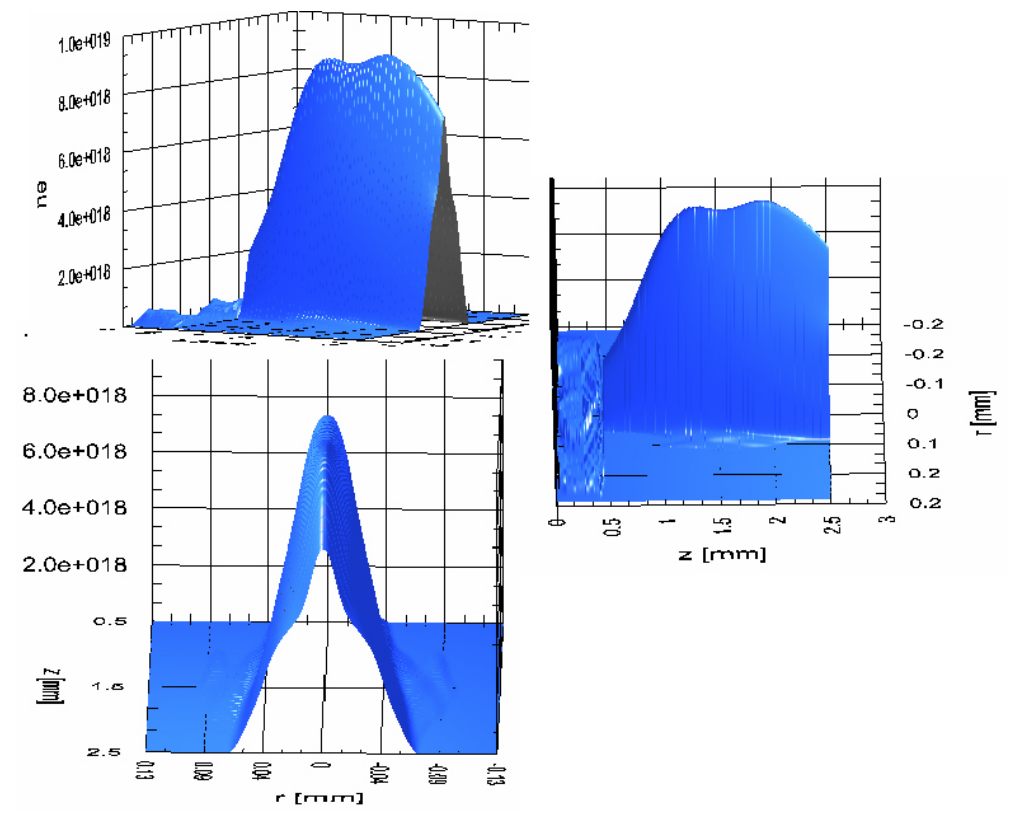

Figure 4.9: Density profile taken on 11/16/05, shot 210.

The peak power of the pump pulse in the simulations was adjusted within the range of uncertainty of the actual pump power in order to match the transverse size of the doubly-ionized region of the Helium plasma between the simulation and the measurement. This was done in conjunction with adjusting the focused spot size within a few percent of the measured value of $17.6 \mu \mathrm{m}$, in order to match the curvature observed in the $30 \mathrm{TW}$ case. The plateau of the simulated density profile was also adjusted to match the periodicity observed in the wake measurement. Having done this, the agreement in the general features of the wake structure is excellent. The relative transverse dimensions of the wake compared to that of the doubly-ionized Helium plateau, the flatness of the wakes in the $10 \mathrm{TW}$ case, and the curvature of the wakes in the $30 \mathrm{TW}$ case, and the progression of curvature in the latter case all match quite well between the simulation and the measurement. 


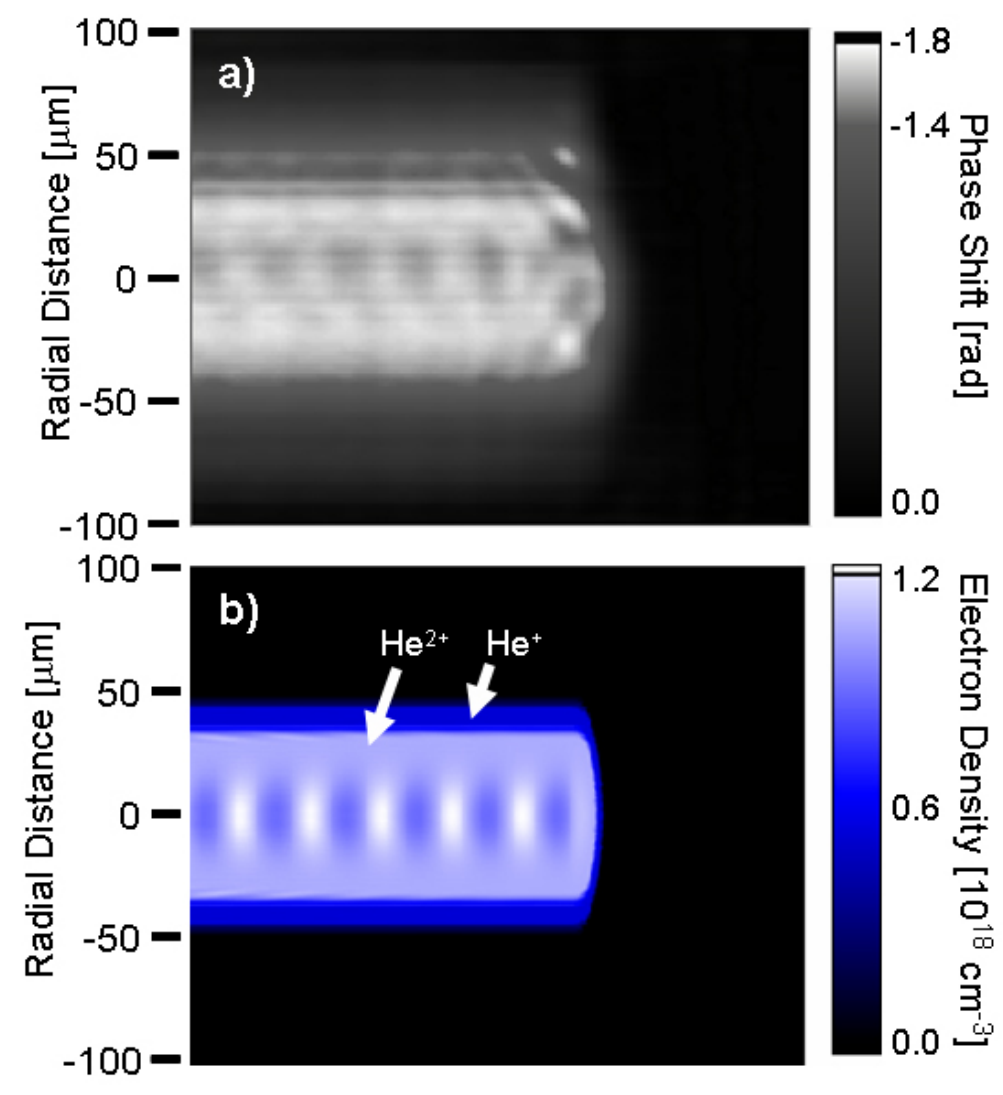

Figure 4.10: a) shot 048 from 11/21/05, and b) $11 \mathrm{TW}$ simulation in trapezoidal density profile with plateau at $0.95 \times 10^{18} \mathrm{~cm}^{-3}$.

\subsubsection{Wakefield Amplitude}

Given that the general shape of the wakefields in the simulations match to those in the measurements, the next most important aspect of correspondence is the amplitude. In other words, do the measured amplitudes $\delta n_{e} / n_{e}$ agree with those predicted by simulation? Figure 4.12 shows this comparison:

Clearly, there is a significant discrepancy in the amplitude of the wakes as inferred from the measured phase shift, and that retrieved from simulations with corresponding experimental parameters. There are a variety of physical considerations 


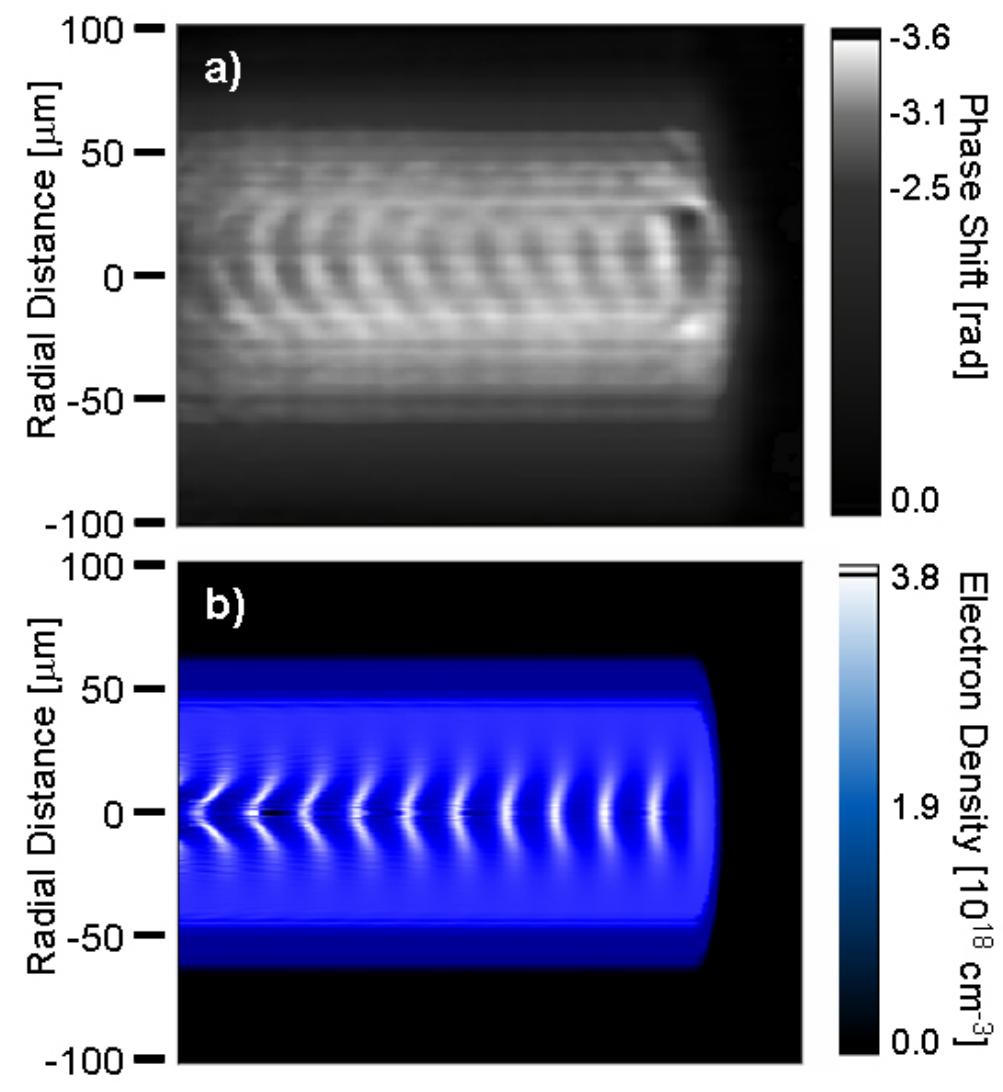

Figure 4.11: a) shot 022 from $11 / 22 / 05$, and b) 40 TW simulation in trapezoidal density profile with plateau at $2.17 \times 10^{18} \mathrm{~cm}^{-3}$.

that can contribute to this discrepancy. The most important of these are:

- Group Velocity Walk-off: induces a shift in the relative positions of the probe and the wake structure, causing a blurring of the image.

- Pump-Probe Walk-off: the group velocity of the pump and probe pulses are different in the plasma due to difference in optical wavelength.

- Variation in group velocity of probe from peak to trough of wake (especially in the nonlinear case), due to dependence of group velocity on local density. 

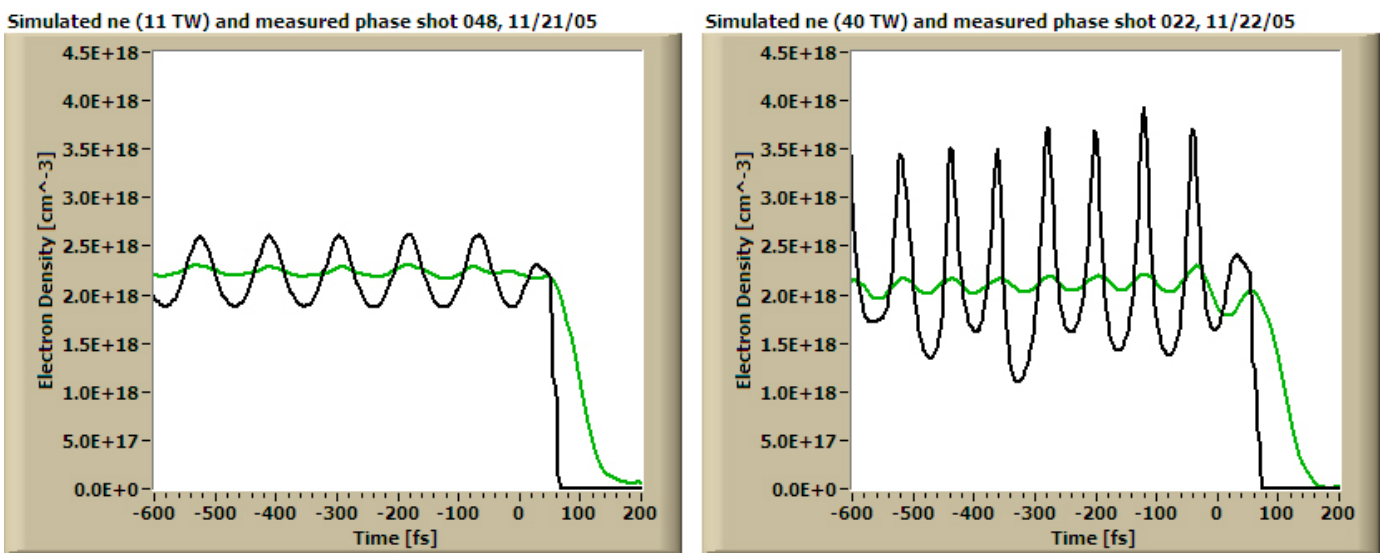

Figure 4.12: Comparison of the wake amplitude measured in shot 048, 11/21/05 (left) and in shot $022,11 / 22 / 05$ (right) to the amplitudes in corresponding simulations.

- Non-density contributions to refractive index of plasma.

- Relativistic motion of electrons in the wave contributes to a relativistic mass increase of electrons (gamma), decreasing the effective refractive index.

- Relativistic electron motion also contributes to index of refraction through the $\vec{v} \times \vec{B}$ term in the electron response to the probe.

- Evolution of the wake structure through the interaction

- Variation in the laser spot-size through the focus causes the instantaneous pump peak power to increase and decrease, inducing a variation in the wake amplitude

- Variations in the plasma density from jet non-uniformities cause the plasma wavelength to vary through the interaction

- Evolution of the wakefield through interaction with the pump pulse, the electron beam or itself can cause the wake structure to significantly 
change as during wave-breaking, pump-depletion or beam-loading.

The issue of group velocity walk-off between the pump and the probe limits the range of densities in which wakefields can be measured by FDH when the pump and probe have different wavelengths. For this experiment, with the pump at 800 $\mathrm{nm}$ and the probe at $400 \mathrm{~nm}$, the walk-off after $2 \mathrm{~mm}$ of interaction in the plasma is about $1 / 10$ of a plasma wavelength at a density of $2 \times 10^{18} \mathrm{~cm}^{-3}$, and about 1 whole plasma wavelength at a density of $2 \times 10^{19} \mathrm{~cm}^{-3}$. For the densities employed here, the group walk-off is thus not likely to be the most important effect. At least, it doesn't explain the extent of the discrepancy between the amplitudes inferred from measurement, and those calculated by simulation. By-passing for the moment, bullet 2, the third principal bullet addresses question Q1 above. The essential point is that the Frequency Domain Holography diagnostic does not actually produce a measurement of an instantaneous wake structure at any location. Rather, as has been described above, the diagnostic produces an image depicting the total phase shift accrued by the probe pulse co-propagating with a wake structure through the interaction. This wake structure can potentially be evolving through the interaction point. Thus it is important to distinguish the measured phase image, which is an integrated result, from the wake structure shown in the simulation, which is an instantaneous density profile (in this case taken from the center of the profile). To better understand the relationship between these two images, the WAKE simulation was modified to simulate the probe, (i.e. to include the effect of the density fluctuations on a co-propagating probe pulse). The effect on the probe pulse is calculated by accounting for the relevant quantities of local electron density and velocity in computing the local instantaneous index of refraction. In this approach, there is no back-effect of the probe on the plasma. The first noticeable thing about the simulation of the probe phase shift is that the morphology is considerably different from that of the instantaneous electron density. Figures 4.13 and 4.14 show the results 
for the low and high power cases, respectively.
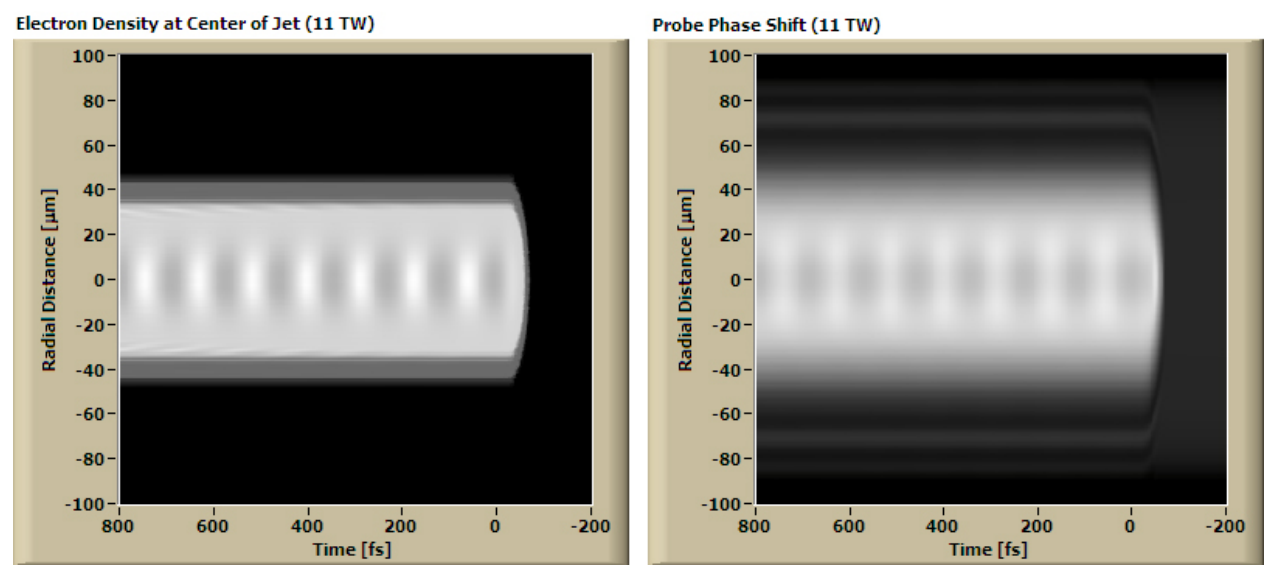

Figure 4.13: Simulated density (left) and phase shift (right) for 11 TW case.
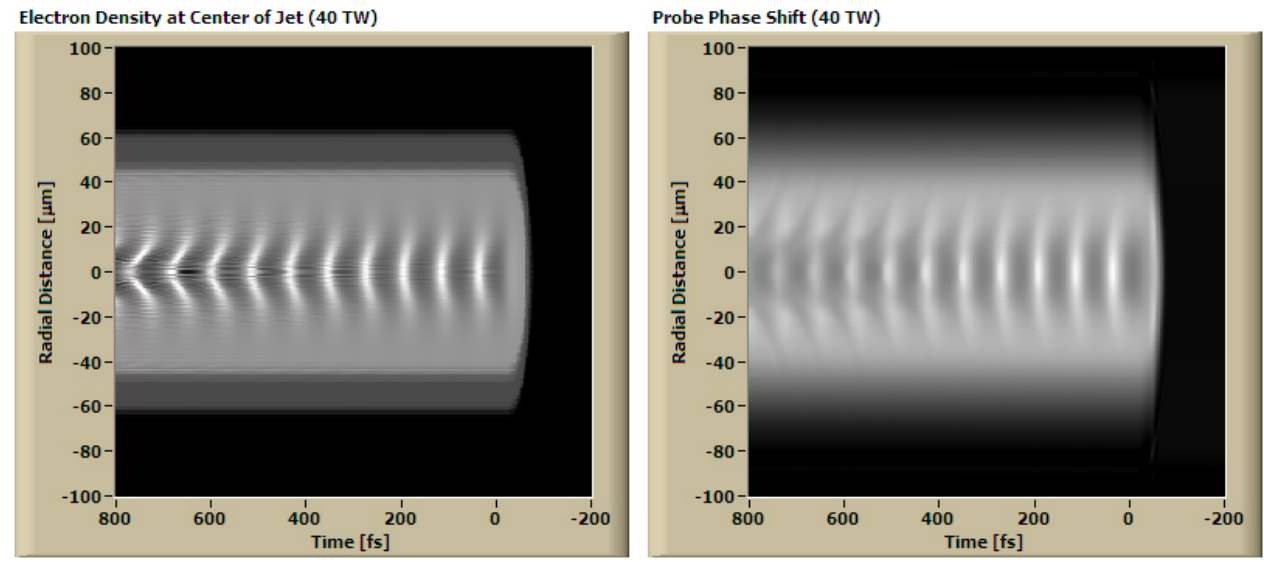

Figure 4.14: Simulated density (left) and phase shift (right) for 40 TW case.

Generally, there appears to be an averaging of the structure, as is somewhat expected. Radially sharp features become blurred in part through natural diffraction of the probe, as described in section 2.5.4. The question of importance, now, is whether the simulated probe pulses come close to describing the phase shifts observed in the measured ones. Comparing the density profile inferred from the sim- 
ulated probe to that of the measured probe, we see that there is still a significant discrepancy between them (fig. 4.15).
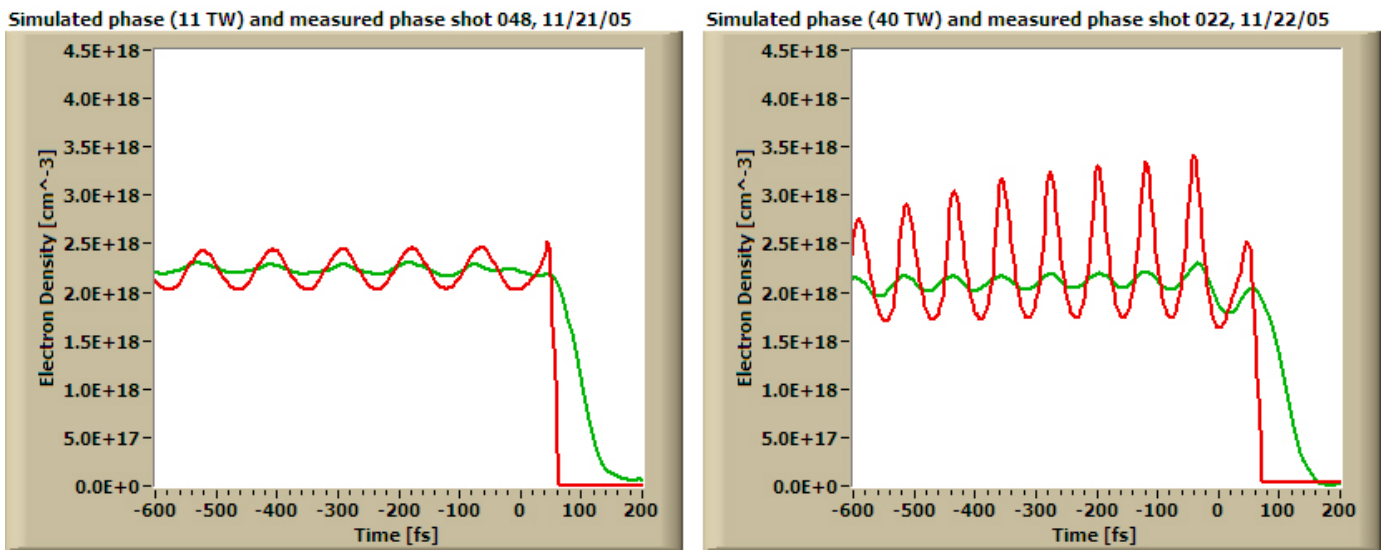

Figure 4.15: Comparison of the wake amplitude measured in shot 048, 11/21/05 (left) and in shot $022,11 / 22 / 05$ (right) to the amplitudes recovered from the simulated probe phase shift.

If the electron density inferred from the simulated phase shift is compared directly to the calculated electron density at the center of the jet, it is clear that there is some difference, though much less than that noted between the simulated probe and measured probe (fig. 4.16).

\subsubsection{Density gradients in the gas profile}

In order to have better agreement between the results of the simulated probe and those of the measured probe, it is necessary to more closely approximate the actual conditions of the experiment. One of the obvious next steps is to include a more realistic gas-density profile in the simulation. As indicated in principal bullet \#3, a longitudinal variation in the density of the gas jet causes the local plasma wavelength to vary during the propagation. In the frame of the probe pulse, a uniform plasma density produces wave structures that remain fixed during the propagation and can 

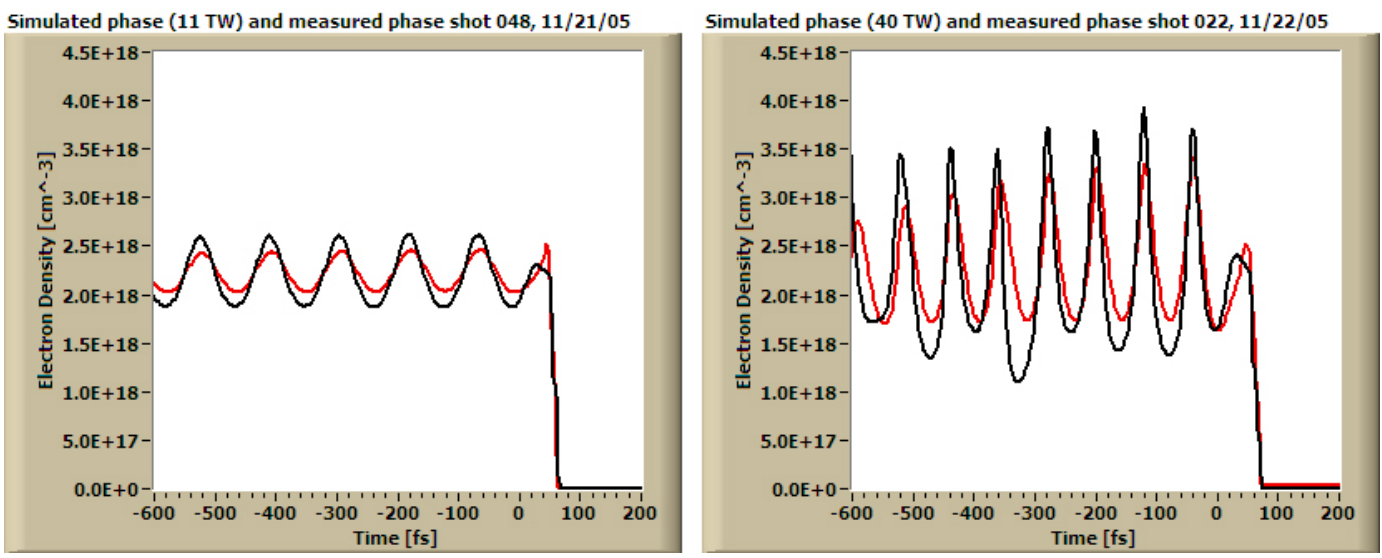

Figure 4.16: Comparison of the wake amplitudes calculated at the center of the jet to the amplitudes extrapolated from the simulated probe for the low (left) and high (right) power cases.

be "photographed" with a long exposure time. A density gradient, on the other hand, causes the waves to lengthen and contract during the propagation, blurring the exposure. Regions of high gradient will then cause a greater degree of blurring, (or averaging), and will consequently contribute less to the overall wake signal, while regions of low gradient will contribute a correspondingly greater amount. The ionization front, however, has a profile which is relatively insensitive to density, except in its amplitude. Therefore, the ionized column will contribute a phase shift throughout the entire interaction, regardless of jet-density profile. What this means is that the shape of the density profile can strongly influence the relative magnitude of the measured plasma oscillations compared to the background level of ionized gas, making the wakefields appear artificially small.

Fortunately, measurement of the actual density profile experienced on a given shot was made possible by the transverse probe and the shearing interferometer. Figure 4.17 shows lineouts of the longitudinal density profiles measured for shot 048 on $11 / 21 / 05$ and shot 022 on $11 / 22 / 05$.

The simulations were then redone using $4^{\text {th }}$ order polynomial fits to the actual 

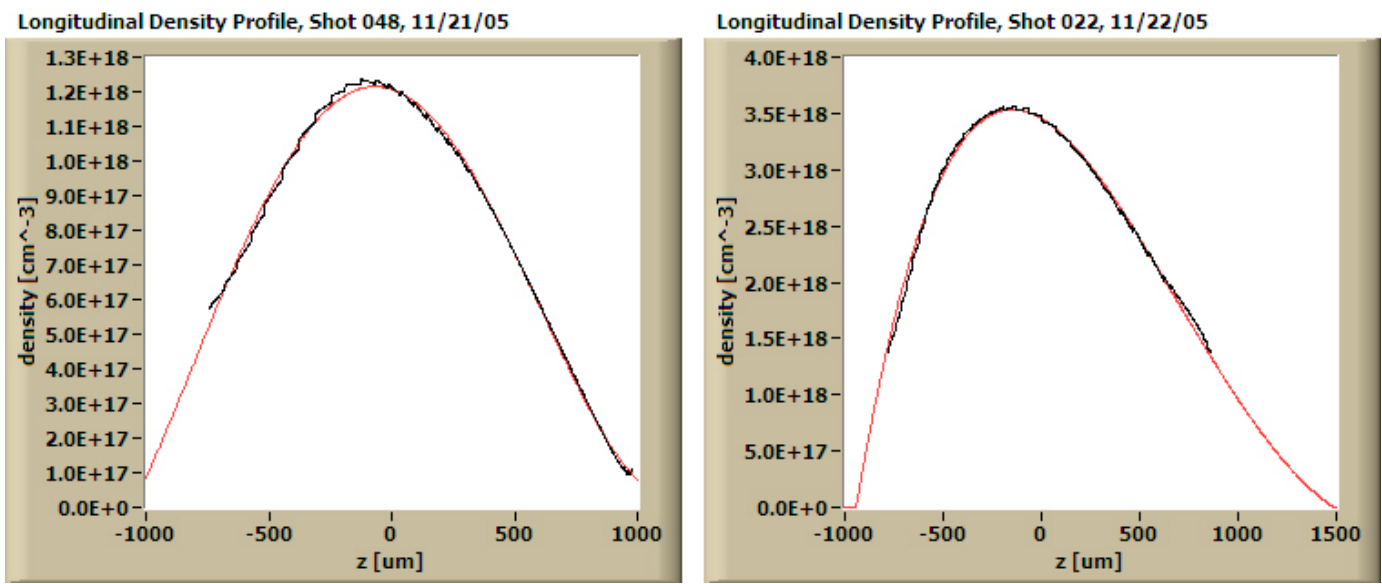

Figure 4.17: Lineouts of the measured longitudinal density profiles for shots 048 on $11 / 21 / 05$ and 022 on $11 / 22 / 05$. 4th order polynomials were used to fit them.

measured profiles. The comparison between the simulated probe phase shift and the measured probe phase shifts is now much more favorable, at least in the case of the higher power data (fig. 4.18).

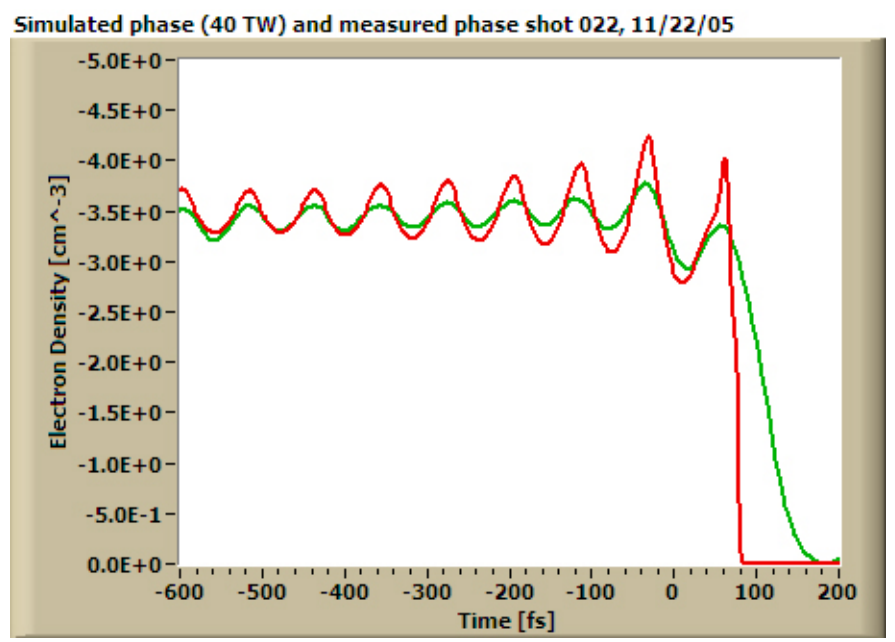

Figure 4.18: Lineout comparison between the measured phase shift and the simulated probe phase shift (40 TW case) using a realistic longitudinal gas-density profile. 
If the pump pulse peak power in the simulation is reduced to the $30 \mathrm{TW}$ of nominal power that was expected in the experiment, then the agreement is even better (fig. 4.18). Thus we can see that the electron-density gradients generated in the actual profile of the gas jet are clearly having a significant effect on the measurement. What is also clear, is that the wake profile that is recovered using this technique approximates fairly closely the structure of the wakefields as they exist at the peak of the density profile. Longitudinal averaging then effectively erases the contributions to the structure from the regions of the gas jet where the gradients are strong.

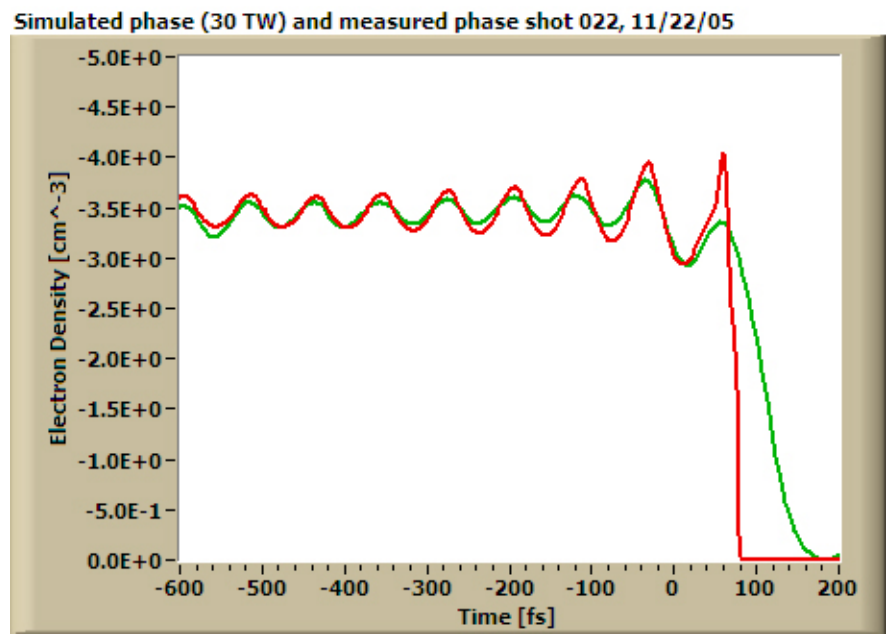

Figure 4.19: Lineout comparison between the measured phase shift and the simulated probe phase shift (30 TW case) using a realistic longitudinal gas-density profile. 


\section{Chapter 5}

\section{Conclusions and Future Directions}

In this dissertation, the first images of the morphological structure of individual wakefields have been produced. The images were taken in a single-shot, real-time mode. The results show a high degree of detail in radial and longitudinal structure of the wakefields, including curvature of the wave fronts, and variation in the wake amplitude with laser peak power. The ability to measure these wakefields, and to be able to do so in real time represents a significant advance in the field of Wakefield acceleration physics. Though there are some shortcomings in the current implementation of this technique, there is much promise for being able to modify it for a variety of important applications, including as a diagnostic for wakefield structure in a "plasma afterburner" experiment. These experiments, being performed at the Stanford Linear Accelerator Center (SLAC), hold some of the greatest promise for use of wakefield acceleration. This work has only scratched the surface of wakefield metrology, and it is expected that future work will address many of the current issues regarding the amplitude discrepancy. Longitudinal averaging, for example, may be studied by the use of machined plasma profiles with high aspect entrance and exit 
regions, and uniform plateaus, controlling the interaction region very precisely. This work and many others will have to be the subject of another dissertation.

\section{$5.1 \quad$ potential modifications}

- Use probe wavelengths comparable to the driving wavelength to avoid group velocity walk-off

- Use a pump and probe that intersect the interaction region at a small angle to reduce the region of overlap. This addresses longitudinal averaging.

- Use a very small f/number for the probe beam to localize the longitudinal region over which phase information is retrieved. This addresses longitudinal averaging.

- Use gas jet profiles that have been "machined" to produce very sharp edges. This will allow restriction of the interaction region to small zones and addresses the longitudinal averaging issue. 


\section{Bibliography}

[1] T. Tajima and J. Dawson, "Laser Electron Accelerator," Phys. Rev. Lett., 43, 267-270 (1979)

[2] S. Mangles et al., "Monoenergetic beams of relativistic electrons from intense laser plasma-intercations," Nature, 431, 535-538 (2004)

[3] C.G.R. Geddes et al., "High-quality electron beams from a laser wakefield accelerator using plasma-channel guiding," Nature, 431, 538-541 (2004)

[4] F. Faure et al., "A laser-plasma accelerator producing monoenergetic electron beams," Nature, 431, 541-544 (2004)

[5] M. Kalal and K. A. Nugent, "Abel inversion using fast Fourier transforms" Appl. Opt., 27, 1956-1959 (1988)

[6] , J.-R. Marques et al., "Laser wakefield: experimental studies of nonlinear radial oscillations", Phys. Plasmas 10, 1124-1134 (1998) 


\section{Vita}

Nicholas Hill Matlis was born in Sterling, Scotland on August $3^{\text {rd }}$, 1972, the son of Eben Matlis and Margarita Josefina Hill Secco de Matlis. After graduating from Evanston Township High School in 1990, he attended Northwestern University in Evanston, Illinois, where his father is a Professor Emeritus of Mathematics. He received the degree of Bachelor of Arts from Northwestern University in 1994, one of three students to ever graduate with a quadruple major. In August of 1994 he entered the Graduate School of The University of Texas at Austin, where he has held positions as T.A., A.I., R.A. and finally R.E.S.A.

Permanent Address: 2501 Wickersham Ln. \#2012

Austin, TX 78741

This dissertation was typeset with $\mathrm{IAT}_{\mathrm{E}} \mathrm{X} 2 \varepsilon^{1}$ by the author.

\footnotetext{
${ }^{1} \mathrm{LTT}_{\mathrm{E}} \mathrm{X} 2_{\varepsilon}$ is an extension of $\mathrm{LT}_{\mathrm{E}} \mathrm{X}$. $\mathrm{LAT}_{\mathrm{E}} \mathrm{X}$ is a collection of macros for $\mathrm{T}_{\mathrm{E}} \mathrm{X}$. $\mathrm{T}_{\mathrm{E}} \mathrm{X}$ is a trademark of the American Mathematical Society. The macros used in formatting this dissertation were written by Dinesh Das, Department of Computer Sciences, The University of Texas at Austin, and extended by Bert Kay, James A. Bednar, and Ayman El-Khashab.
} 